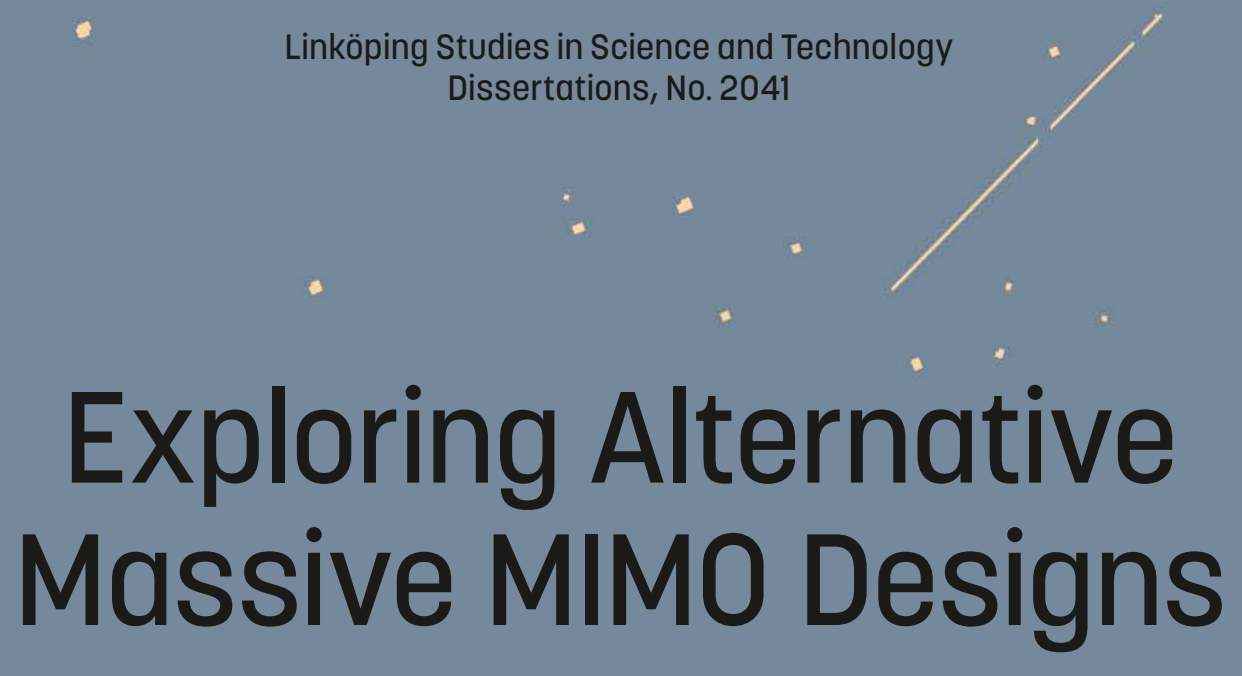

\title{
Superimposed Pilots and Mixed-ADCs
}

Daniel Verenzuela
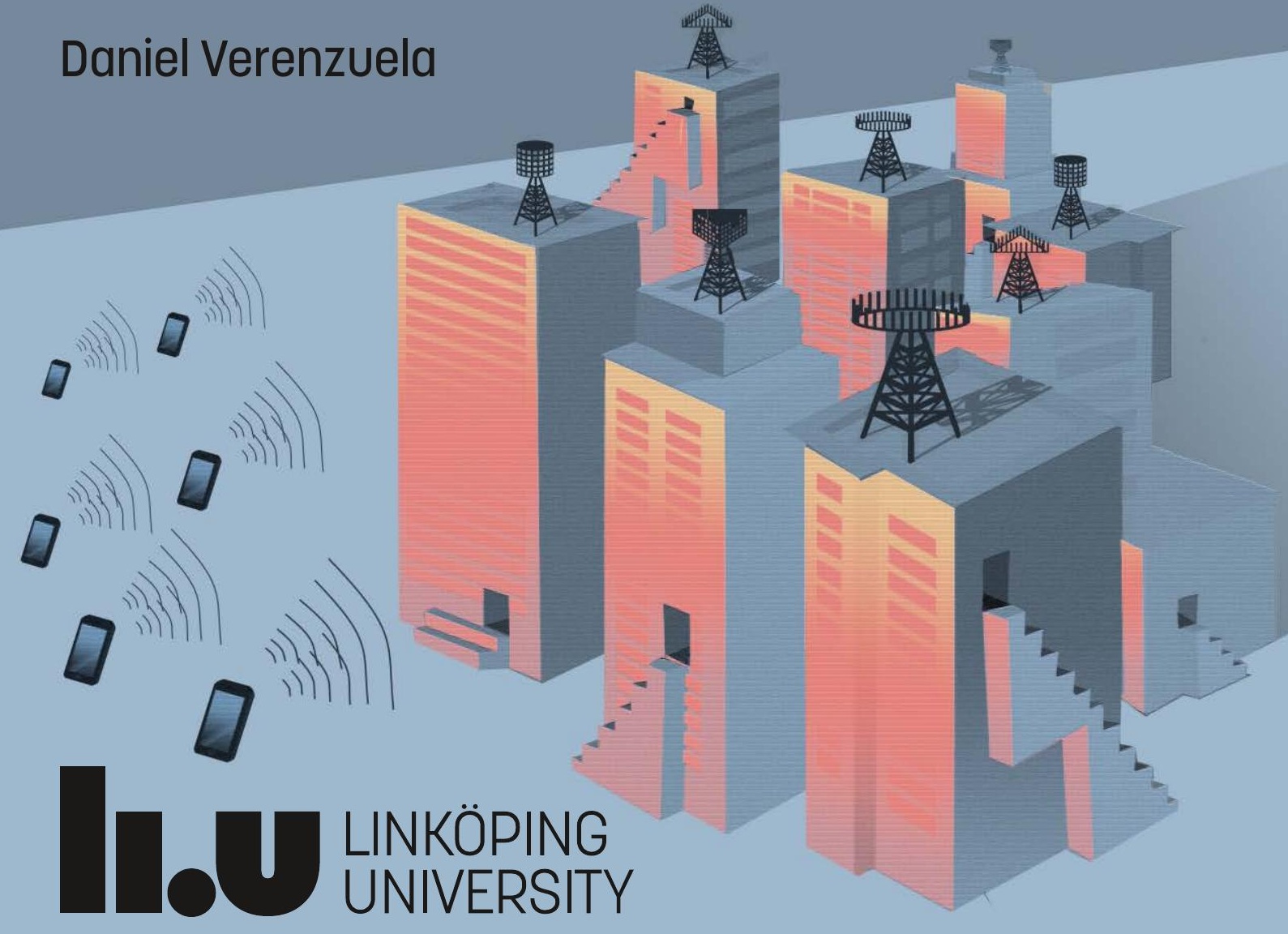
Linköping Studies in Science and Technology

Dissertations, No. 2041

\title{
Exploring Alternative Massive MIMO Designs \\ Superimposed Pilots and Mixed-ADCs
}

\author{
Daniel Verenzuela
}

\section{COMMUNICATION SŶYSTEMS}

Division of Communication Systems Department of Electrical Engineering (ISY) Linköping University, 58183 Linköping, Sweden www.commsys.isy.liu.se 
This is a Swedish Doctor of Philosophy Dissertation.

The Doctor of Philosophy degree comprises 240 ECTS credits of postgraduate studies.

Cover: A Massive MIMO network where the stairs in the buildings represent the different ADC bit resolutions in a mixed-ADC design. The two merging signals from cellular phones illustrate the superposition of pilot and data signals, corresponding to the superimposed pilots design. This cover was painted by Andrea Britto Moreno with design input from the author.

Exploring Alternative Massive MIMO Designs

(C) 2020 Daniel Verenzuela, unless otherwise noted. ISBN 978-91-7929-921-7

ISSN 0345-7524

Printed in Sweden by LiU-Tryck, Linköping 2020 


Para Jeannine e Isabella 



\section{Abstract}

The development of information and communication technologies (ICT) provides the means for reaching global connectivity that can help humanity progress and prosper. This comes with high demands on data traffic and number of connected devices which are rapidly growing and need to be met by technological development. Massive MIMO, where MIMO stands for multiple-input multiple-output, is a fundamental component of the $5 \mathrm{G}$ wireless communication standard for its ability to provide high spectral and energy efficiency, SE and EE, respectively. The key feature of this technology is the use of a large number of antennas at the base stations (BSs) to spatially multiplex several user equipments (UEs).

In the development of new technologies like Massive MIMO, many design alternatives need to be evaluated and compared in order to find the best operating point with a preferable tradeoff between low cost and complexity. In this thesis, two alternative designs for signal processing and hardware in Massive MIMO are studied and compared with the baseline operation in terms of SE, EE, and power consumption. The first design is called superimposed pilot (SP) transmission and is based on superimposing pilot and data symbols to eliminate the need to reserve dedicated time-frequency resources for pilots. This allows more data to be transmitted and supports longer pilot sequences that, in turn, reduce pilot contamination. The second design is mixed analog-to-digital converters (ADCs) and it aims at balancing the SE performance and the power consumption cost by allowing different ADC bit resolutions across the BS antennas.

The results show that the Massive MIMO baseline, when properly optimized, is the preferred choice in standard deployments and propagation conditions. However, the SP alternative design can increase the SE compared to the baseline by using the Massive-MIMO iterative channel estimation and decoding (MICED) algorithm proposed in this dissertation. In particular, the SE gains are found in cases with high mobility, high carrier frequencies, or high number of spatially multiplexed UEs. For the mixed-ADCs alternative design, improvements in the SE and EE compared to the Massive 
MIMO baseline can be achieved in cases with distributed BS antennas where interference suppression techniques are used. 


\section{Resumen}

El desarrollo en tecnologías de información y comunicación (en inglés, ICT) provee los medios para alcanzar la conectividad global que puede ayudar a la humanidad a progresar y prosperar. Esto implica que el avance tecnológico debe satisfacer la alta demanda de tráfico de data y número de equipos conectados que se encuentra en rápido crecimiento. La tecnología de múltiple-entrada múltiple-salida masiva, en inglés Massive MIMO, se considera una pieza fundamental de la quinta generación de comunicaciones inalámbricas (5G) debido a su capacidad de proveer una alta eficiencia espectral y energética (en inglés, SE y EE, respectivamente). Esta tecnología está caracterizada fundamentalmente por el uso de un alto número de antenas en la estación base (en inglés, BS) para multiplexar a varios usuarios en el espacio.

En el desarrollo de nuevas tecnologías como Massive MIMO, muchas alternativas de diseño necesitan ser evaluadas y comparadas para encontrar el mejor punto de operación con un balance conveniente entre complejidad y bajo costo. En esta tesis, dos alternativas de diseño para el procesamiento de señales y el hardware de Massive MIMO son estudiadas y comparadas con la operación del diseño base en términos de eficiencia espectral, eficiencia energética y consumo de potencia. El primer diseño se denomina transmisión de pilotos superpuestos (en inglés, SP) y está basado en la superposición de señales piloto y de datos para eliminar la necesidad de asignar recursos dedicados a señales pilotos. Además, la transmisión de pilotos superpuestos permite reducir la interferencia que surge a raíz de reusar las señales pilotos en distintas celdas, este efecto se denomina contaminación de pilotos (en inglés pilot contamination). El segundo diseño se denomina conversores analógico-adigital (en inglés, ADC) mixtos (en inglés, mixed-ADCs) y se basa en permitir distintas resoluciones de bit en los conversores analógico-a-digital de las antenas en la estación base. Este diseño permite que la resolución de los conversores analógico-a-digital se adapte a las condiciones de propagación de las señales para balancear los beneficios en eficiencia espectral con el costo de potencia consumida. 
Los resultados muestran que el diseño base de Massive MIMO, cuando esta optimizado de manera apropiada, es la opción preferida en despliegues y condiciones de propagación estándares. Sin embargo, la transmisión de pilotos superpuestos puede incrementar la eficiencia espectral en comparación al diseño base cuando se combina con el método iterativo para la estimación de canal y decodificación en Massive MIMO propuesto en esta tesis (en inglés, MICED). En particular, las ganancias en eficiencia espectral son obtenidas en escenarios con alta movilidad de usuarios, alta frecuencia portadora, o alto número de usuarios multiplexados en el espacio. Con respecto al diseño alternativo de conversores analógico-a-digital mixtos, la eficiencia espectral y energética pueden ser incrementadas en comparación al diseño base cuando las antenas de la estación base están distribuidas en el espacio y técnicas para suprimir interferencia entre usuarios son usadas. 


\section{Zusammenfassung}

Die Entwicklung der Informations- und Kommunikationstechnologien (ICT) bietet die Möglichkeit eine globale Konnektivität zu erreichen, die Fortschritt und Wohlstand fördern kann. Dies bedeutet zugleich, dass der steigende Datenverkehr und die wachsende Anzahl verbundener Geräte eines entsprechenden technologischen Fortschritts bedarf. Massive MIMO, wobei MIMO für multiple-input multiple-output steht, ist eine fundamentale Komponente des drahtlosen 5G Kommunikationsstandards, da sie eine hohe spektrale Effizienz (SE) und Energieeffizienz bietet (EE). Die Hauptkomponente dieser Technologie ist die Nutzung einer großen Anzahl an Antennen auf Seiten der Basisstationen (BSs) um mehrere Nutzer zu bedienen, die ihre Signale zur selben Zeit auf derselben Frequenz senden während sie in der räumlichen Domäne getrennt sind (spatial multiplexing).

In der Entwicklung neuer Technologien wie Massive MIMO müssen viele Designalternativen evaluiert und verglichen werden um den optimalen Betriebspunkt im Sinne eines sinnvollen Gleichgewichts zwischen Kosteneffizienz und Komplexität zu finden.

In dieser Doktorarbeit werden zwei alternative Designs für Signalverarbeitung und Hardware in Massive MIMO Systemen untersucht und in Bezug auf spektrale Effizienz, Energieeffizienz und Stromverbrauch mit dem Massive MIMO Basisdesign verglichen. Das erste Design heißt überlagerte Pilotton Übertragung (superimposed pilot, SP) und basiert auf der Überlagerung von Pilotton und Datensignal, damit nicht mehr die Notwendigkeit besteht bestimmte Ressourcen für Pilottöne zu reservieren. Dies ermöglicht die Übertragung größerer Datenmengen und reduziert die Interferenz, die aus der wiederholten Nutzung der Pilottöne in verschiedenen Zellen resultiert (pilot contamination). Das zweite Design nennt sich gemischte analog zu digital Konverter (mixed analog-to-digital converters, ADCs) und erlaubt es einen Kompromiss zwischen hoher spektraler Effizienz und niedrigem Stromverbrauch zu finden. Dies geschieht indem die Bit Auflösung an jeder BS Antenne an die Ausbreitungsbedingungen der Signale angepasst wird.

Die Resultate zeigen, dass das Massive MIMO Basisdesign, wenn es rich- 
tig optimiert ist, bei Standardeinsätzen und unter normalen Ausbreitungsbedingungen, die bevorzugte Wahl ist. Das alternative SP Design kann jedoch die spektrale Effizienz im Vergleich zum Basisdesign durch die Nutzung des in dieser Dissertation vorgeschlagenen Massive MIMO iterativen Kanalschätzungs- und Dekodierungsalgorithmus (MICED) erhöhen. Die verbesserte spektrale Effizienz findet sich insbesondere in Fällen hoher Nutzermobilität, hoher Frequenzen oder hoher Anzahl an gleichzeitig bedienter Nutzer. Das gemischte analog zu digital Konverter Design ermöglicht in Fällen verteilter Basisstationen bei denen Interferenz unterdrückende Techniken genutzt werden eine verbesserte spektrale Effizienz und Energieeffizienz. 


\section{Populärvetenskaplig sammanfattning}

Utvecklingen av informations- och kommunikationsteknik (IKT) gör det möjligt för människor från hela världen att kopplas samman och utbyta kunskaper. Ju mer vi vet och förstår om varandra, desto större är chansen att mänskligheten kan uppnå globala utvecklingsmål och välstånd.

IKT-utvecklingen är associerad med höga krav på datatakter och antal uppkopplade enheter. Dessa krav ökar ständigt och måste mötas med teknologisk utveckling. Massiv MIMO, där MIMO står för multiple-input multiple-output, är flerantennteknik och en grundsten i nästa generations trådlösa kommunikationssystem. Huvudanledningen till detta är att tekniken kan förbättra spektraleffektiviteten (SE), vilket är ett mått på hur väl vi kan kommunicera data över begränsade radiofrekvensresurser. Tekniken förbättrar även energieffektiviteten (EE), vilket är ett mått på hur effektivt tekniken använder energi till att kommunicera data.

Massiv MIMO bygger på användandet av ett stort antal av antenner på basstationerna för att kommunicera med ett flertal användare samtidigt och på samma frekvensresurser. Detta möjliggörs genom "rumslig multiplexing" vilket betyder att signaler från användare på olika platser kan separeras på basstationen i den rumsliga domänen. Denna separering kräver att basstationen först mäter egenskaperna hos signaler som kommer från de olika användarnas positioner.

När en ny teknik, såsom Massiv MIMO, utvecklas är det viktigt att olika alternativa designer utvärderas och jämförs för att identifiera den bästa varianten. Detta kan exempelvis vara den variant som uppnår en viss balans mellan hög kommunikationsprestanda och låg kostnad. I denna avhandling utvärderas två alternativa sätt att designa signalbehandlingen och hårdvaran i Massiv MIMO. Dessa jämförs med konventionell Massiv MIMO i termer av SE, EE och effektförbrukning.

Den första alternativa designen kallas överlagrade piloter och bygger på 
att kända pilotsignaler och okända datasignaler skickas samtidigt från användarna, istället för efter varandra. Pilotsignalerna används för att mäta upp de trådlösa kanalerna som signalerna färdas över medan datasignalerna innehåller den information som ska kommuniceras. Genom att överlagra pilotsignalerna så behövs inga dedikerade radioresurser för piloter och därmed finns det mer resurser för datasändning. Dessutom minskar överlagrandet de störningar som kommer från andra användare som använder samma pilot, vilket kallas pilotkontaminering.

Den andra alternativa designen kallas mixade analog-till-digital (AD) omvandlare. En $\mathrm{AD}$-omvandlare är en krets som behövs på varje antenn för att omvandla analoga radiosignaler till digitala signaler som kan processas i en dator. Bitupplösningen i AD-omvandlaren avgör hur många nivåer som kan användas för att representera den analoga signalen. Ju högre bitupplösning desto fler nivåer och därmed en mer noggrann representation, men detta leder även till högre beräkningskomplexitet och effektförbrukning. Mixade AD-omvandlare försöker balansera mellan hög prestanda och låg komplexitet genom att optimera bitupplösningen på varje antenn i ett Massiv MIMO system.

Avhandlingens resultat visar att det går att öka SE i Massiv MIMO genom att använda överlagrade piloter, ifall den föreslagna algoritmen MICED (Massive-MIMO iterative channel estimation and decoding) används. Förbättringarna är särskilt stora när användarna har hög mobilitet, när en hög bärfrekvens används eller när antalet rumsligt multiplexade användare är högt. När det gäller mixade AD-omvandlare så kan små förbättringar i SE uppnås, jämfört med konventionell Massiv MIMO, när bitupplösningen i $\mathrm{AD}$-omvandlarna optimeras under förutsättning att signalstyrkan varierar mellan basstationens antenner.

Sammanfattningsvis så kan de alternativa designerna av Massiv MIMO som studerats i avhandlingen ge små prestandaförbättringar jämfört med konventionella metoder. Men trots detta så kan de konventionella metoderna uppnå en bra avvägning mellan hög prestanda och låg komplexitet ifall de optimeras väl. 


\section{Acknowledgments}

The path through academic research is filled with many obstacles, detours and even traps (e.g., in the form of long mathematical derivations that turn out to be intractable). Thus, to navigate successfully through the deep waters of academic research it is of paramount importance to have a trusting and unwavering guidance. It is an honor for me to have professor Emil Björnson as my academic supervisor and mentor, without whom, this work would not have been possible. His mentoring enabled me to develop as a researcher and to accomplish tasks ranging from solving difficult mathematical problems to creating and pursuing my own problem formulations and research interests. His expert advice extends from general matters such as finding research directions and explaining complicated theories with ease; to the deep specifics such as finding key insights in long mathematical expressions and that ever elusive typo. His vast knowledge and expertise in many different areas, his commitment towards his students, his dedication and passion towards excellence, have been a beacon that has guided and inspired me to be the researcher that I am today, I am truly grateful for his tutoring.

As a $\mathrm{PhD}$ student, it is a privilege to work in a group that has the highest standards of academic research. Therefore, I would like to thank professor Erik G. Larsson for giving me the opportunity to join his research group. His expert advice, teaching and commitment to high quality research have been fundamental for my development as a researcher.

Obtaining expert advise and feedback from diverse sources is essential to become a researcher. I am fortunate to have as co-supervisors professor Luca Sanguinetti (University of Pisa) and professor Michail Matthaiou (Queen's University Belfast). I thank professor Luca Sanguinetti for providing expert feedback, helping me navigate through difficult reviews, and encouraging further collaborations. I thank professor Michail Matthaiou for sharing his expert knowledge on complicated hardware topics, and giving advice and feedback on my research. Their dedication and support have helped me achieve the results that are found in this dissertation.

During my doctoral studies, I had the opportunity to conduct a research 
visit to the institute of telecommunications at Stuttgart University. I would like to thank professor Stephan ten Brink for hosting me at his research group, and for broadening my horizon with his expert advice and vast knowledge. I would also like to thank Xiaojie Wang and Maximilian Arnold for their enthusiasm to exchange ideas and continuously improve our research. Their dedication to achieve excellence and provide meaningful contributions to state of the art research, have helped me grow as a researcher. In addition, I would like to thank all my colleagues at the institute of telecommunications at Stuttgart University for providing a great work environment.

The never ending discussions and debates on research directions, complicated homework problems, basic theory concepts, and new publications, among others, have all been part of my education in research, and I thank my colleagues from the division of Communication Systems at Linköping University (LiU) from 2015 until 2020 for sponsoring this essential component of my education. Furthermore, I have found great friends in my time as a $\mathrm{PhD}$ student at LiU, who have made this journey one of the best experiences one could hope for.

Family is, and has always been, at the core of my motivation to pursue excellence. They provide the never ending support and encouragement for me to be the best I can be in all aspects of life. I dedicate this dissertation to: my parents, Juan y Coro; my brothers, Juan y David; my big family, "los Verenzuela-Moreno"; and last but not least my loving wife, Jeannine, and beloved daughter, Isabella, who inspire me to be better every day.

Daniel Verenzuela Linköping, January 2020 


\section{Contents}

$\begin{array}{lll}\text { Notation } & \text { xxi }\end{array}$

Abbreviations $\quad$ xxiii

Introduction $\quad 1$

1 Motivation $\quad 3$

1.1 Interference Management with Imperfect CSI . . . . . . . 6

1.2 Hardware Design . . . . . . . . . . . . . 8

1.3 Contributions of this Dissertation .......... . 11

1.4 Included Papers . . . . . . . . . . . . . . . . . 12

1.5 Excluded Papers ................. 15

2 Massive MIMO $\quad 17$

2.1 Origins of Massive MIMO . . . . . . . . . . . . . 17

2.2 Communication Channel . . . . . . . . . . . 19

2.3 Properties of Massive MIMO Channels . . . . . . . 23

2.3.1 Stochastic Channel Model . . . . . . . . . . 25

2.3.2 Impact of Spatial Correlation . . . . . . . . . . 27

2.4 Channel Estimation . . . . . . . . . . . . 29

2.5 Capacity Analysis . . . . . . . . . . . . . 35

2.6 Performance of Massive MIMO . . . . . . . . . . . 38

3 Alternative Massive MIMO Designs $\quad 47$

3.1 Superimposed Pilots . . . . . . . . . . . . 48

3.1.1 LMMSE Channel Estimation . . . . . . . . . . . 49

3.1.2 Spectral Efficiency ............. 51

3.1.3 Impact of Power Control in the UL . . . . . . . . . 61

3.1.4 The MICED Algorithm . . . . . . . . . . . . 65

3.2 Hardware Impairments Optimization . . . . . . . . . 77

3.2.1 Parametrized HWIs Model . . . . . . . . . 77 
3.2.2 Optimal Tradeoff between HWIs and BS Antennas . . 82

3.2.3 Optimal HWI Allocation under i.i.d. Rayleigh Fading 85

3.2.4 Optimal HWI Allocation under Correlated Rayleigh Fading and LSF Variation among BS Antennas . . . . 89

4 Summary, Conclusions, and Future Work

A Spectral and Energy Efficiency of Superimposed Pilots in Uplink Massive MIMO

1 Introduction . . . . . . . . . . . . . . . . . . 121

2 Network Model . . . . . . . . . . . . . . . . . . . . . . . 124

3 Channel Estimation . . . . . . . . . . . . . . . 126

3.1 Regular Pilots . . . . . . . . . . . . . 126

3.2 Superimposed Pilots . . . . . . . . . . . . . . . 128

4 Achievable Rates with MRC . . . . . . . . . . . . . . . . . . . 129

4.1 Random Pilot Allocation . . . . . . . . . . . . 130

4.2 Regular Pilots . . . . . . . . . . . . . . . . 131

4.3 Superimposed Pilots . . . . . . . . . . . . . . . 133

5 Analysis of Achievable Rates . . . . . . . . . . . . . . 138

6 Performance Comparison under Random Deployment . . . . 140

6.1 Energy Efficiency Modeling . . . . . . . . . . . . . . 143

7 Numerical Results . . . . . . . . . . . . . . . . . . . . . 144

8 Conclusions . . . . . . . . . . . . . . . . . . . . 149

References ....................... 155

B Joint UL and DL Spectral Efficiency Optimization of Superimposed Pilots in Massive MIMO 159

1 Introduction . . . . . . . . . . . . . . . . . . . 161

2 System Model . . . . . . . . . . . . . . . . . . 163

3 Channel Estimation . . . . . . . . . . . . . . . 164

4 DL Achievable Rates . . . . . . . . . . . . . . . . . . . . 166

5 SE Comparison between RPs and SPs . . . . . . . . . . 169

$5.1 \quad$ Multiobjective Optimization with RPs and SPs . . . 170

6 Numerical Results . . . . . . . . . . . . . . . . . . . . . . 172

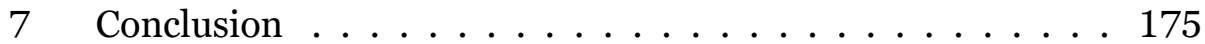

References ...................... 176 
C Optimal Power Control for Superimposed Pilots in Uplink Massive MIMO Systems

1 Introduction . . . . . . . . . . . . . . . . . 183

2 System Model . . . . . . . . . . . . . . . . . . . . . . . 184

3 Channel Estimation . . . . . . . . . . . . . . . . . 185

4 Achievable SE with MRC . . . . . . . . . . . . . . . 186

5 Optimal Power Control with SP . . . . . . . . . . . . . . 188

$5.1 \quad$ Max-min Fairness . . . . . . . . . . . . . . . . . 189

5.2 Max-product of SINRs . . . . . . . . . . . . . 189

6 Statistical Channel Inversion Power Control . . . . . . . . . 190

7 Numerical Results . . . . . . . . . . . . . . . . . . . . . . 191

8 Conclusions . . . . . . . . . . . . . . . . . . 194

References . . . . . . . . . . . . . . . . . . 194

D Massive-MIMO Iterative Channel Estimation and Decoding (MICED) in the Uplink 197

1 Introduction . . . . . . . . . . . . . . . . . 199

1.1 Contributions . . . . . . . . . . . . 201

2 System Model . . . . . . . . . . . . . . . . . . . 203

3 Uplink Combining and Achievable SE . . . . . . . . . . . . 207

4 MICED - Massive-MIMO Iterative Channel Estimation and

Decoding . . . . . . . . . . . . . . . . 209

4.1 Basis of the MICED Algorithm . . . . . . . . . . . 209

4.2 Analysis of Data-Aided Channel Estimation with Gaussian Symbols . . . . . . . . . . . . . . 211

4.3 Computational Complexity . . . . . . . . . . . . 215

4.4 Numerical Example with Gaussian Symbols . . . . . 216

5 Finite alphabet symbols . . . . . . . . . . . . . . . 220

$5.1 \quad$ Numerical Examples with Finite-Alphabet Symbols . 223

6 Conclusion . . . . . . . . . . . . . . . . . 227

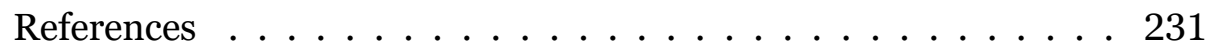

E Hardware Design and Optimal ADC Resolution for Uplink Massive MIMO Systems 237

1 Introduction . . . . . . . . . . . . . . . . . 239

2 System Model . . . . . . . . . . . . . . . . . . . . 240

3 Achievable Rate with Imperfect CSI . . . . . . . . . . . . 242

3.1 Power Consumption Model . . . . . . . . . . . . . 244

4 Optimal Hardware Impairments Tradeoff . . . . . . . . . . . 246

5 Numerical Results . . . . . . . . . . . . . . . . . . . . 247

6 Conclusion . . . . . . . . . . . . . . . . 248 


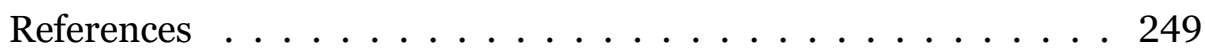

F Per-Antenna Hardware Optimization and Mixed Resolution ADCs in Uplink Massive MIMO 253

1 Introduction . . . . . . . . . . . . . . . . . 255

2 System Model . . . . . . . . . . . . . . . . 256

3 Channel Estimation . . . . . . . . . . . . . . 257

4 Achievable SE . . . . . . . . . . . . . . . . 259

5 Effect of Mixed ADC Resolution _. . . . . . . . . . . 260

5.1 Impact on Achievable SE . . . . . . . . . . . . 260

5.2 Impact on Power Consumption . . . . . . . . . . . 263

6 Numerical Results . . . . . . . . . . . . . . . . . . 263

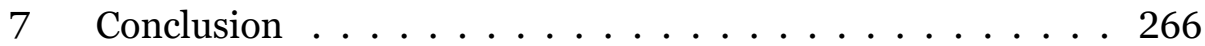

References ........................ 266

G Optimal Per-Antenna ADC Bit Allocation in Correlated and Cell-Free Massive MIMO $\quad 269$

1 Introduction . . . . . . . . . . . . . . . 271

2 System Model . . . . . . . . . . . . . . . . . . 274

3 Channel Estimation . . . . . . . . . . . . . . 276

3.1 HWI Allocation for Minimal Pilot Distortion . . . . . 278

3.2 Integer ADC Bit Allocation . . . . . . . . . . . 279

3.3 Numerical Example . . . . . . . . . . . . . . . . 280

4 Achievable SE . . . . . . . . . . . . . . . . 282

4.1 Numerical Example with Minimum Pilot Distortion . 285

5 Optimal HWI Allocation . . . . . . . . . . . . . . . 286

5.1 Maximizing the SINR . . . . . . . . . . . . . . 287

6 Impact of Power Consumption on the HWI Allocation . . . . 292

7 Conclusion . . . . . . . . . . . . . . 295

References . . . . . . . . . . . . . . . . . 298 


\section{Notation}

This is a summary of the basic notation used in the introduction of this dissertation. Note also that additional terms are presented and defined along the text. Matrices are denoted by upper-case boldface letters (e.g., X) and column vectors are denoted by lower-case bold face letters (e.g., $\mathrm{x}$ ).

The following notation is used:

$\mathbb{C} \quad$ Set of complex numbers

$\mathbb{R} \quad$ Set of real numbers

$\mathbb{N} \quad$ Set of positive integer numbers

$|\cdot| \quad$ Absolute value operator

$\|\cdot\| \quad$ Euclidean norm operator

$(\cdot)^{*} \quad$ Conjugate operator

$(\cdot)^{T} \quad$ Transpose operator

$(\cdot)^{H} \quad$ Conjugate transpose operator

$(\cdot)^{\dagger} \quad$ Pseudo inverse (or Moore-Penrose inverse) operator

$\log _{2}(\cdot) \quad$ Logarithm function with base 2

$\operatorname{abs}(\cdot)^{2} \quad$ Element-wise squared absolute value operator

$\operatorname{det}(\cdot) \quad$ Determinant operator

$\operatorname{tr}(\cdot) \quad$ Trace operator

$\mathbf{I}_{M} \quad$ Identity matrix of size $M \times M$

$[\mathbf{X}]_{i j} \quad$ Element in row $i$ and column $j$ of matrix $\mathbf{X}$

$[\mathbf{X}]_{j} \quad$ The $j^{\text {th }}$ column of matrix $\mathbf{X}$

$[\mathbf{x}]_{j} \quad$ Element $j$ of column vector $\mathbf{x}$

$[\mathbf{X}]_{i: j} \quad$ Sub-matrix formed by the columns $[\mathbf{X}]_{n}$ such that $n \in\{i, i+1, \ldots, j-1, j\}$ with $i<j$

$\mathbb{E}\{\cdot\} \quad$ Expected value operator

$\mathbb{E}\{\cdot \mid x\} \quad$ Expected value operator conditioned on $x$ 
$\operatorname{Var}\{\cdot\} \quad$ Variance operator

$\operatorname{Pr}(\cdot) \quad$ Probability operator

$\mathcal{C N}\left(0, \sigma^{2}\right) \quad$ Circularly symmetric complex Gaussian distribution with variance $\sigma^{2}$

$\mathcal{U}(a, b) \quad$ Uniform distribution on the interval $[a, b]$ 


\section{Abbreviations}

\begin{tabular}{ll} 
3GPP & 3rd Generation Partnership Project \\
ADC & Analog-to-Digital Converter \\
BER & Bit Error Rate \\
BLER & Block Error Rate \\
BS & Base Station \\
CSI & Channel State Information \\
DL & Downlink \\
DSP & Digital Signal Processing \\
EE & Energy Efficiency \\
EVD & Eigen Value Decomposition \\
FDD & Frequency Division Duplexing \\
flops & floating point operations per second \\
FoM & Figure-of-Merit \\
HWI & Hardware Impairment \\
ICT & Information and Communication Technologies \\
i.i.d. & independent identically distributed \\
LDPC & Low Density Parity Check \\
LMMSE & Linear Minimum Mean Squared Error \\
MICED & Massive-MIMO Iterative Channel Estimation and Decoding \\
MIMO & Multiple-Input Multiple-Output \\
mMMSE & multicell Minimum Mean Squared Error \\
MMSE & Minimum Mean Squared Error \\
MR & Maximum Ratio \\
MSE & Mean Squared Error \\
MU-MIMO & Multiuser Multiple-Input Multiple-Output \\
NR & New Radio \\
PPP & Poisson Point Process \\
QAM & Quadrature Amplitude Modulation \\
QPSK & Quadrature Phase Shift Key \\
RF & Radio Frequency \\
\hline &
\end{tabular}




$\begin{array}{ll}\text { RP } & \text { Regular Pilot } \\ \text { RZF } & \text { Regularized Zero Forcing } \\ \text { HWI } & \text { Hardware Impairment } \\ \text { SE } & \text { Spectral Efficiency } \\ \text { SER } & \text { Symbol Error Rate } \\ \text { SIMO } & \text { Single-Input Multiple-Output } \\ \text { SINR } & \text { Signal-to-Interference-plus-Noise Ratio } \\ \text { SISO } & \text { Single-Input Single-Output } \\ \text { S-MMSE } & \text { Singlecell Minimum Mean Squared Error } \\ \text { SNR } & \text { Signal-to-Noise Ratio } \\ \text { SP } & \text { Superimposed Pilot } \\ \text { TDD } & \text { Time Division Duplexing } \\ \text { UE } & \text { User Equipment } \\ \text { UL } & \text { Uplink } \\ \text { ZF } & \text { Zero Forcing }\end{array}$




\section{Introduction}





\section{Chapter 1}

\section{Motivation}

Since the beginning of the 21st century there has been a massive expansion of information and communication technologies (ICT) all over the world. The Internet has become an essential part of our lives in developed societies and it has brought information and tools for progress to developing countries. In particular, wireless communications allow access to information and connectivity in highly mobile situations as well as in remote locations. The possibility to access information and have means of communication in a global scale brings us closer together and may help humanity to further develop and prosper.

To achieve global connectivity, mobile communication systems need to accommodate a massive amount of data traffic and number of connected devices while having low energy consumption. The mobile data traffic is expected to increase at an exponential rate for the foreseeable future and by the early 2020's there will be 1.5 mobile devices per person in the world, most of which will be smartphones demanding high data rates $[1,2]$. To meet the growing demand, the throughput of mobile networks needs to be increased by orders of magnitude [3]. On the other hand, the energy consumption and carbon footprint of the ICT industry is becoming an important societal and environmental concern $[4,5]$. This means that the throughput needs to increase without incrementing the energy consumption of mobile networks, which calls for orders of magnitude improvements in energy efficiency (EE).

In cellular networks, the total throughput is defined as

$$
\mathrm{R}[\mathrm{bit} / \mathrm{s}]=\underbrace{N_{\mathrm{CELL}}}_{\text {Number of cells }} \times \underbrace{B_{\mathrm{W}}[\mathrm{Hz}]}_{\text {Bandwidth }} \times \underbrace{\mathrm{SE}[\mathrm{bit} / \mathrm{s} / \mathrm{Hz} / \text { cell }]}_{\text {Spectral efficiency }},
$$

and to improve it; either more cells, more bandwidth, or higher spectral efficiency (SE) is needed. Adding more cells, known as cell densification, 


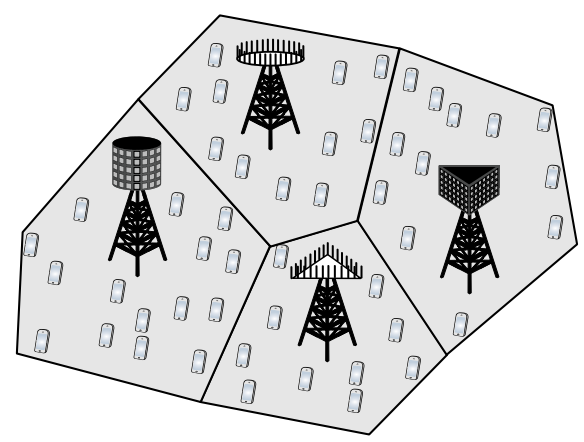

Figure 1: Multicell Massive MIMO system.

has been the main source of throughput improvement over the past decades. However, as cells are placed closer together, interference increases up to the point in which the throughput saturates and starts to decrease [6]. On the other hand, the bandwidth is limited by the amount of radio frequency spectrum that is available. Increasing the SE is a viable way of improving the throughput, however it comes at the cost of adding more complexity into the network which may result in higher power consumption. This dissertation focuses on increasing the SE while considering its impact on the power consumption. The total EE of a cellular network is defined as

$$
\mathrm{EE}[\mathrm{bit} / \mathrm{Joule}]=\frac{N_{\mathrm{CELL}} \times B_{\mathrm{W}}[\mathrm{Hz}] \times \mathrm{SE}[\mathrm{bit} / \mathrm{s} / \mathrm{Hz} / \mathrm{cell}]}{\text { Power consumption }[\mathrm{Joule} / \mathrm{s}]},
$$

which means that reducing the power consumption and improving the SE results in a EE increase. Other ways to increase EE can be to both increase the SE and the power consumption with the SE growing at a faster pace, or to decrease both the power consumption and the SE with the power consumption decreasing at a faster pace. However, these latter options are not the main focus of this dissertation.

In the development and implementation of $5 \mathrm{G}$ wireless communication systems, the Massive MIMO technology, where MIMO stands for multipleinput multiple-output, it a fundamental component for its ability to provide both high SE and EE $[7,8]$.

\section{What is Massive MIMO?}

In this dissertation, Massive MIMO is defined as a cellular multiuser MIMO (MU-MIMO) technology with a massive number of antennas and user equipments (UEs), as illustrated in Figure 1. More precisely, each base station 
(BS) is equipped with a large number of antennas and utilizes these antennas to spatially multiplex many UEs on the same time-frequency resource. The main difference from conventional cellular networks is that the interference between UEs is managed by spatial processing (called transmit precoding or receive combining) instead of by scheduling them on orthogonal timefrequency resources. The key differentiator from conventional MU-MIMO systems is that the users' channels become nearly spatially orthogonal-a property referred to as "favorable propagation"-when the BS has a large number of antennas (See Chapter 2 for a detailed definition). This property vastly reduces the interference and thus allows for spatial multiplexing of many tens of UEs, instead of a handful UEs. In addition, favorable propagation enables the use of relatively simple linear signal processing techniques for interference suppression [7,8]. The main advantages of Massive MIMO are (see Chapter 2 for a more detailed explanation):

\section{Spatial multiplexing.}

The BSs separate the signals received from (and transmitted to) the UEs in the spatial domain such that multiple parallel data streams can be supported at the same time and in the same frequency band. This results in a linear increase of the SE per cell proportional to the number of served UEs.

\section{Coherent processing gain.}

The BSs process the signals received from (and transmitted to) each antenna element coherently. Here, coherent signal processing, means that the phase and amplitude of signals received from (or transmitted to) each antenna element are processed so that they add constructively when they are received from (or at) desired points in space where the UEs are located. This translates into a logarithmic gain in the SE per UE with respect to the number of BS antennas. Due to the large number of BS antennas, high SE per UE can be supported.

\section{Transmission power reduction.}

Thanks to the coherent processing gain, the transmission power can be scaled down proportionally to the square root of the number of BS antennas while still increasing the SE.

\section{Linear processing.}

The large number of BS antennas induces two properties in the channels between the BS and the UEs called asymptotic favorable propagation and channel hardening. These properties allow the use of linear processing techniques that simplify the implementation while providing high SE. 


\section{Low end hardware.}

The coherent processing allows the gain of the desired signals to increase with the number of BS antennas whereas in most cases the distortions caused by hardware imperfections are combined non-coherently. Since there are many BS antennas, the hardware quality of each antenna branch can be relaxed, reducing the power consumption while supporting high SE, and in turn, high EE.

In the development and implementation of any new technology, there are always challenges that need to be surpassed and Massive MIMO is no exception. This leads to many different design proposals that need to be assessed and compared in a rigorous and fair manner to find the best possible system design. While [7] describes the baseline Massive MIMO system design, this dissertation explores alternative designs that have been proposed to overcome some of the challenges of this technology.

\subsection{Interference Management with Imperfect CSI}

In Massive MIMO, performing coherent signal processing is essential. This can be achieved by obtaining channel state information (CSI) from pilot transmission or by performing blind channel estimation and data detection. The latter is an interesting field of study, however, these methods often have high complexity and require both large time-frequency resources and number of BS antennas to perform well [9-11]. Thus, this dissertation is focused on the former, that is, estimating the channels between the BS and served UEs based on the transmission of known pilot sequences. These pilots allow the BS to identify and separate the channels from different UEs and perform channel estimation. This method requires some time-frequency resources to be reserved for pilot sequences, called pilot overhead, which reduces the available resources for data. In this dissertation, a time division duplexing (TDD) protocol is assumed in which pilot sequences are transmitted by the UEs in the uplink (UL) from which the BS estimates the channels to the UEs. These channel estimates are then used to process the signals in the UL and downlink (DL) by virtue of channel reciprocity $[7,8]$.

The communication channel is continuously changing both in time and frequency. Thus, the estimation and communication procedures need to happen in a short time and frequency block where the channel can be considered to be time invariant and frequency flat. This time-frequency block is called the coherence block and it contains a limited number of information symbols (see Chapter 2 for more details). This means that the number of available 
pilot sequences is limited, and in a multicell system, pilots need to be reused among cells. When UEs share the same pilot sequence, the channel estimates obtained at the BS are contaminated by interference which diminishes the estimation quality and reduces the SE $[7,8]$. This effect is called pilot contamination, and in uncorrelated channels, it leads to interfering terms that scale proportionally to the number of BS antennas.

There is a wide literature on mitigating the pilot contamination effect, as an example $[7,8,12-18]$. One approach, is to use the spatial correlation between the signals received at different BS antenna elements to separate UEs with the same pilot, as done in [8,12-14]. Another approach, is to increase the pilot overhead and allow for longer pilot sequences [7,15-18]. In a multicell system, the longer the pilot sequences, the less often they need to be reused in the network, thus the pilot contamination effect is reduced by the increased pathloss between UEs sharing the same pilot. However, having a larger pilot overhead reduces the number of symbols available for data transmission. The tradeoff between reducing the pilot contamination and having low pilot overhead is studied in [17], concluding that a pilot overhead between $5 \%$ and $40 \%$ maximizes the sum throughput.

In the aforementioned works, the transmission of pilot and data symbols is done separately within the coherence block to reduce interference in the channel estimation process. This method is known in the literature as regular pilot (RP) transmission. An alternative method is to simultaneously transmit a superposition of pilot and data symbols, which is referred to as superimposed pilot (SP) transmission and it is studied in [19-25] for the case of a single-cell system. This method allows the use of the whole UL part of the coherence block for both pilots and data simultaneously. Improvements in bit-error-rate (BER) and mean squared error (MSE) of channel estimates are found in $[21,22]$ for SP in comparison to RP.

Recently, [23-25] have shown promising results in multicell Massive MIMO systems with SP. The main idea is that UEs transmit a linear combination of pilot and data symbols within the whole UL part of the coherence block. This allows the use of longer pilot sequences without having a pilot overhead. Hence, pilot contamination is reduced and the SE may be improved in comparison to RP. However, sending pilot and data symbols simultaneously leads to interference in the channel estimation process from data symbols. This degrades the estimation quality and creates correlation between channel estimates and data, which in turn, decreases the SE gain.

In Paper A, the performance of SP in the UL of a multicell Massive MIMO system is evaluated and compared with RP. To this end, novel rigorous closedform rate expressions are derived with SP. This stands in contrast to prior works, [23, 25], which evaluate SP using approximate expressions of signal- 
to-interference-plus-noise ratios (SINRs) and the MSEs of the channel estimates. In Paper B, RP and SP are compared in a multiobjective optimization framework where the UL and DL are jointly optimized for maximal sum SE. In Paper C the optimal power allocation with SP is studied based on the closed-form rate expressions are derived in Paper A.

In summary, RP channel estimation provides sufficiently accurate CSI to obtain high SINR, which translates into high SE in Massive MIMO. However, this comes at the expense of having a pilot overhead, which in turn, limits the maximum achievable SE. On the other hand, the results in Papers A and B show that SP channel estimation gives comparable SE to that of RP and it is instead limited by the data interference. Thus, a potential way to further improve the SE in Massive MIMO is to use SP with data-aided channel estimation to reduce the data interference.

The idea of using partially decoded data to improve channel estimation with SP has been proposed a couple of decades ago for single antenna communication systems [19]. Extensions to single-user point-to-point MIMO systems [26,27] show an improvement in terms of BER with SP, which translates into higher SE compared to RP since SP removes the pilot overhead. In the case of Massive multiuser MIMO, [23, 25, 28, 29] show that iterative data-aided channel estimation with SP has the potential to increase the SE compared to RP. However, evaluating the effect of channel coding in a multicell Massive MIMO system is lacking. In addition, theoretical analysis on the impact of spatial correlation, inter-user and intercell interference is missing in the aforementioned literature.

In Paper D, the Massive-MIMO Iterative Channel Estimation and Decoding (MICED) algorithm is proposed to harvest the benefits of data-aided channel estimation in multicell Massive MIMO systems. To obtain insights into the benefits of the MICED algorithm, closed-form expressions for the error correlation matrices of data-aided channel estimates are computed assuming Gaussian distributed data symbols. Furthermore, numerical analysis with quadrature phase shift keying (QPSK) modulation and low-density parity check (LDPC) codes is used to evaluate the performance of the MICED algorithm compared to conventional pilot-based channel estimation in terms of the block-error-rate (BLER) and the achievable SE.

\subsection{Hardware Design}

In cellular networks up to $4 \mathrm{G}$, the BSs account for the largest portion of power consumption [30], and within macro BSs, the power amplifiers and site cooling are responsible for over $80 \%$ of this consumption [31]. By using 
a large number of BS antennas, Massive MIMO can substantially reduce the transmission power in comparison to $3 \mathrm{G}$ and $4 \mathrm{G}$ macro BSs $[7,8]$. As a result, the power amplifiers would require much less power to operate, and in turn, the cooling requirements would also be reduced since the power amplifiers are the main responsible of heat dissipation.

However, increasing the number of BS antennas comes with a higher deployment cost and circuit power consumption. Thus, to implement the Massive MIMO technology, the circuit cost and power consumption per antenna need to be significantly reduced in comparison to 3G and 4G BS's circuitry. This calls for low-complexity hardware at each antenna's radio frequency (RF) chain which is the chain of circuit components that are needed to transmit and receive data. However, reducing the hardware complexity introduces additional distortion into the system, degrading the SE. Fortunately, the large number of BS antennas is able to mitigate most of these effects by non-coherent distortion combining and significant gains in SE and EE can still be obtained with Massive MIMO [32,33]. Thus, there is a tradeoff in the number of BS antennas and hardware quality to achieve high SE and low power consumption.

An important source of distortion and power consumption in a Massive MIMO BS is the analog-to-digital converters (ADCs) which are responsible for sampling and quantizing the analog UL signals; and mapping them into digital signals. The number of quantization levels is given by the ADC bit resolution such that the distortion decreases whereas the power consumption and complexity increase with the ADC bit resolution. In [34-37], the selection of the ADC bit resolution is studied to maximize performance in terms of BER, SE, and power consumption, showing that Massive MIMO can provide high SE with low-resolution ADCs. Here, low ADC bit resolution refers to values of up to 4-5 bits which is considerably lower than standard ADCs used in $3 \mathrm{G}$ and 4G BS deployments which have around 15 bits. ${ }^{1}$ The works $[36,38]$ show that Massive MIMO can even operate with one-bit ADCs. However, it is not clear whether operating with such low hardware precision is preferable in terms of SE and EE.

The design of a wireless network entails weighing different performance metrics that are often mutually conflicting. In particular, this situation arises when maximal SE and minimal power consumption are desired. This tradeoff has been studied in $[39,40]$ for single-antenna and millimeter wave MIMO systems, respectively. In Paper E, the selection of the number of BS antennas

\footnotetext{
${ }^{1}$ The use of such high ADC resolution is mainly done to combat the large variations between received power from different UEs, and out-of-band interference. In Massive MIMO, these effects can be mitigated by power control and the coherent signal processing with large number of BS antennas.
} 
and the ADC bit resolution per antenna is optimized for maximal SE under a power consumption constraint.

In all the aforementioned works, all RF chains have equal ADC bit resolution. Alternatively, a mixed-ADC approach has been proposed in [41,42] where the ADC bit resolution is allowed to be different among BS antennas and vary at high speed following the fast changes in the wireless channel. In addition, $[42,43]$ study the optimal $\mathrm{ADC}$ bit allocation to maximize SE in millimeter wave and Massive MIMO systems, respectively. These works concluded that mixed-ADCs can achieve significant gains in the SE compared to using only low-resolution ADCs. However, the effect of channel estimation in MU-MIMO detection has not been considered. Recently, [44] studied a mixed-ADC system where a small portion of BS antennas have highresolution ADCs and the rest have 1-bit ADCs, which is the lowest possible resolution. The analysis considers the effect of imperfect CSI for uncorrelated channels, and it concludes that the most benefits of mixed-ADCs are found at high signal-to-noise ratio (SNR) while using interference suppression techniques.

In Paper F, mixed ADCs are studied under imperfect CSI where the ADC bit resolution of each antenna can be set individually but remains static over time. Majorization theory $[45,46]$ is used to study the order preserving properties of the SE and the power consumption with respect to how unequal the ADC bit resolutions are across the BS antennas.

In Paper G, the ADC bit allocation is optimized based on an achievable SE analysis with spatially correlated channels under imperfect CSI. Here, the ADC bit allocation is not static but it follows the channel changes introduced by the movement of UEs and obstacles in a macroscopic scale ${ }^{2}$ or by serving different UEs in the coverage area over time. In contrast to [41-44], where high speed switches are required to follow the fast changes in the wireless channel, this approach allocates the ADC bits at a much slower time scale. This simplifies the implementation and provides the necessary time to conduct further optimization of the ADC bit allocation. A closed-form optimal ADC bit allocation strategy is proposed to minimize the distortion in pilot signals under an ADC bit budget. Then, a closed-form achievable SE is derived as a rigorous lower bound on the capacity. This SE expression is used to maximize the SINR under an ADC or power consumption budget; or minimize the power consumption under an SINR budget.

\footnotetext{
${ }^{2}$ This scale can span variations orders of magnitude larger than the wavelength of propagating signals.
} 


\subsection{Contributions of this Dissertation}

This dissertation explores alternative designs for a Massive MIMO system in terms of signal processing and hardware. The main idea is to conduct a rigorous analysis of these alternative designs and compare them with the baseline approaches to evaluate the potential gains (if any) that can be attained.

The first alternative design is based on SP, where pilot and data symbols are sent simultaneously, instead of separately, to reduce pilot contamination and remove the pilot overhead. The results show that SP is able to reduce the pilot contamination effect at the expense of adding additional interference that limits the SE gain. To overcome this limitation, the MICED algorithm is proposed in which data-aided channel estimation and decoding is used to increase the SE with SP. The highest SE gain of this approach, compared to RP, is found in scenarios with high mobility or high frequencies. In addition, the MICED algorithm with SP enables aggressive spatial multiplexing that facilitates the implementation of other technologies like machine type communication.

The second alternative design is mixed-ADCs, in which each BS antenna is allowed to have a different ADC bit resolution. The results show that for uncorrelated channels, equal ADC bit resolution across the BS antennas maximizes the SE and minimizes the power consumption, under a constraint on the total number of ADC bits. In the case of spatially correlated channels, the benefits of optimizing the ADC bit allocation are determined by the variations in received power among BS antennas. The most benefits in terms of higher SE and EE, are found when interference suppression techniques are used and the BS antennas are distributed in separate locations.

This dissertation is structured in two main parts: an introduction to the research topic and a collection of scientific papers. The introduction is meant to explain the main concepts, assumptions and insights found in the scientific papers and prior work. In Chapter 2, the basic theory and performance metrics for Massive MIMO are explained. The main ideas behind the alternative Massive MIMO designs including system model, main assumptions, performance evaluation, and insights are explained in Chapter 3. Finally, Chapter 4 summarizes the main contributions of this dissertation and presents potential directions for future work.

The main ideas and topics researched in this dissertation have emerged from the combined effort of the first author, supervisor and co-supervisors. The analysis and writing of the papers presented in this dissertation have all been conducted by the first author with the careful guidance and advice from his supervisor and co-supervisors. The first author would like to acknowledge the contributions provided by the supervisor, co-supervisors and other co- 
authors through careful revision of manuscripts and analysis, as well as sharing their expert advice on review processes, research directions, and general knowledge.

\subsection{Included Papers}

\section{Paper A: Spectral and Energy Efficiency of Superimposed Pilots in Uplink Massive MIMO}

Authored by: Daniel Verenzuela, Emil Björnson and Luca Sanguinetti

Published in: IEEE Transactions on Wireless Communications, vol. 17, no. 11, pp. 7099-7115, Nov. 2018.

This paper compares the performance of SP and RP in terms of SE and EE, for the UL of a multicell Massive MIMO network. A rigorous closed-form SE expression with SP is derived assuming a simple linear processing method called maximum ratio (MR) combining. This formula provides valuable insights into identifying all the interference sources, their impact on the SE and their relationship with the other system parameters. Then, stochastic geometry is used to study the effect associated with intercell interference in a large practical network with an irregular BS deployment. Within this setting, a closed-form lower bound on the SE with SP averaged over the UEs' spatial distribution is derived. This provides powerful insights into the interplay of system parameters without requiring the use of heavy numerical simulations. Such lower bounds are then used to compute the EE of the network with both RP and SP taking into account the power consumed by the signal transmission and circuitry operation. Numerical results show that, when both methods are optimized, RP provides comparable SE and EE to SP in practical scenarios.

\section{Paper B: Joint UL and DL Spectral Efficiency Optimization of Superimposed Pilots in Massive MIMO}

Authored by: Daniel Verenzuela, Emil Björnson and Luca Sanguinetti Published in Proceedings of: IEEE Global Communications Conference Workshops (GC Wkshps), Singapore, 2017, pp. 1-7.

This work performs a comparative analysis of RP and SP in Massive MIMO considering the joint SE of UL and DL communications. A rigorous DL lower bound on the capacity with SP is derived, from which the different sources of interference are identified and an analytical comparison between 
$\mathrm{RP}$ and SP is offered. Then, multiobjective optimization theory is used to compare the joint UL and DL SE performance between RP and SP. Numerical results indicate that $\mathrm{RP}$ and SP give comparable $\mathrm{SE}$ when both methods are optimized.

\section{Paper C: Optimal Power Control for Superimposed Pilots in Uplink Massive MIMO Systems}

Authored by: Daniel Verenzuela, Andreas Bergström, and Emil Björnson Published in Proceedings of: 52nd Asilomar Conference on Signals, Systems, and Computers, Pacific Grove, CA, USA, 2018, pp. 499-503.

This work studies the optimal power control strategies in the UL of a Massive MIMO system with SP and MR combining. The optimization objectives are maximum product of SINRs and max-min fairness. The former aims at increasing the aggregated SE performance while the latter seeks to maximize the minimum SE per UE for maximum fairness. These problems are reformulated as geometric programs which allow for efficient implementations. The numerical results indicate that the SE gains from the optimal power control with respect to the heuristic statistical channel inversion power control, are more significant when the interference from pilot symbols is perfectly subtracted from the received signal.

\section{Paper D: Massive-MIMO Iterative Channel Estimation and Decoding (MICED) in the Uplink}

Authored by: Daniel Verenzuela, Emil Björnson, Xiaojie Wang, Maximilian Arnold, and Stephan ten Brink

Published in: IEEE Transactions on Communications.

This paper proposes the MICED algorithm where partially decoded data is used as side-information to improve the channel estimation and increase SE. The analysis shows that users with precise data estimates can help users with poor data estimates to decode. Numerical results with QPSK modulation and LDPC codes show that the MICED algorithm increases the SE and reduces the BLER with RP and SP compared to conventional methods. The MICED algorithm with SP delivers the highest SE and it is especially effective in scenarios with short coherence blocks (e.g., high mobility or high frequencies) or high number of spatially multiplexed UEs. 


\section{Paper E: Hardware Design and Optimal ADC Resolution for Uplink Massive MIMO Systems}

Authored by: Daniel Verenzuela, Emil Björnson and Michail Matthaiou Published in Proceedings of: IEEE Sensor Array and Multichannel Signal Processing Workshop (SAM), Rio de Janerio, 2016, pp. 1-5.

This work focuses on the hardware design for the efficient operation of Massive MIMO systems. A closed-form UL achievable SE expression is derived considering imperfect CSI and hardware impairments. An optimization problem is formulated to maximize the sum SE subject to a constraint on the total power consumption. The optimization variables are the number of BS antennas and the level of hardware impairments per BS antenna. The solution manifests the fundamental tradeoff between the number of BS antennas and level of hardware impairments. For the case where the source of hardware impairments is the quantization distortion from ADCs, numerical results suggest that hardware quality corresponding to 4 or 5 ADC bit resolution maximizes the SE. Moreover, by properly selecting the power consumption constraint, EE is also maximized for these ADC bit resolutions.

\section{Paper F: Per-Antenna Hardware Optimization and Mixed Resolution ADCs in Uplink Massive MIMO}

Authored by: Daniel Verenzuela, Emil Björnson and Michail Matthaiou Published in Proceedings of: 51st Asilomar Conference on Signals, Systems, and Computers, Pacific Grove, CA, 2017, pp. 27-31.

This work studies the selection of per-antenna hardware quality in terms of ADC bit resolution. A new SE expression is derived for MR combining, and majorization theory is used to analyze the order preserving properties of the SE and the power consumption with respect to the per-antenna ADC bit resolutions. That is, given a fixed sum of ADC bits across the antenna array, is it preferable to use an equal-ADC over a mixed-ADC approach? The results show that having equal-resolution ADCs across the antenna array maximizes the SE and minimizes the power consumption. 


\section{Paper G: Optimal Per-Antenna ADC bit Allocation in Correlated and Cell-Free Massive MIMO}

Authored by: Daniel Verenzuela, Emil Björnson and Michail Matthaiou Submitted to IEEE Transactions on Communications.

This article studies the optimal hardware impairment and ADC bit allocation per BS antenna to maximize SE and EE. The impact of spatial correlation with channel variations between the BS antennas is included to evaluate co-located and cell-free Massive MIMO deployments. The results show that in co-located arrays with low channel gain variations among BS antennas, equal ADC bit allocation is optimal. In contrast, cell-free Massive MIMO systems (where the BS antennas are spread in the service area) benefit the most from optimizing the ADC bit allocation when interference suppression techniques are used.

\section{Paper H: Massive MIMO}

Authored by: Daniel Verenzuela and Emil Björnson

Published in: Wiley Encyclopedia of Electrical and Electronics Engineering, pp. 1-12. 2019.

For better readability, this article has been merged with the introduction of this dissertation rather than being included as a separate paper.

This article is an encyclopedia entry that defines Massive MIMO as a cellular MU-MIMO technology. It starts by giving a concise definition of Massive MIMO and main benefits that it brings. Then, it provides a historical recollection of the main contributions from which Massive MIMO was built on. Next, the main properties, advantages and limitations of Massive MIMO are explained and exemplified. Finally, the definition and main characteristics of Massive MIMO are summarized.

\subsection{Excluded Papers}

The author of this dissertation has also co-authored other papers that have not been included in this dissertation because either they are condense versions of the included papers or they are outside the scope of this dissertation. The excluded papers that are presented as follows: 


\section{Condense versions}

D. Verenzuela, E. Björnson and L. Sanguinetti, "Spectral Efficiency of Superimposed Pilots in Uplink Massive MIMO Systems", in The Proceedings of IEEE Global Communications Conference (GLOBECOM), Singapore, Dec. 2017, pp. 1-6.

\section{Out-of-scope}

D. Verenzuela, E. Björnson and L. Sanguinetti, "Optimal Design of Wireless Networks for Broadband Access with Minimum Power Consumption", in The Proceedings of IEEE International Conference on Communications (ICC), Kuala Lumpur, Malaysia, May. 2016, pp. 1-6.

A. Pizzo, D. Verenzuela, L. Sanguinetti and E. Björnson, "Network Deployment for Maximal Energy Efficiency in Uplink with Zero-Forcing", in The Proceedings of IEEE Global Communications Conference (GLOBECOM), Singapore, Dec. 2017, pp. 1-7.

A. Pizzo, D. Verenzuela, L. Sanguinetti and E. Björnson, "Network Deployment for Maximal Energy Efficiency in Uplink with Multislope pathloss", in IEEE Transactions on Green Communications and Networking, vol. 2, no. 3, pp. 735-750, Sept. 2018.

D. Verenzuela and G. Miao, "Scalable D2D Communications for Frequency Reuse » 1 in 5G”, in IEEE Transactions on Wireless Communications, vol. 16, no. 6, pp. 3435-3447, June 2017. 


\section{Chapter 2}

\section{Massive MIMO}

This chapter introduces the baseline design of Massive MIMO, as well as, the main system model assumptions and performance metrics used for its analysis in this dissertation. In Section 2.1, a historical recollection of the main contributions that gave rise to Massive MIMO is presented. In Section 2.2, the communication channel is defined. Section 2.3 explains the basic properties of Massive MIMO channels. Section 2.4 introduces the process of channel estimation. In Section 2.5, main results on the capacity analysis are presented. Finally in Section 2.6, the main performance metrics used in this dissertation are introduced, that is, SE, EE and power consumption. In addition, a summary of the main benefits and characteristics of Massive MIMO is provided.

\subsection{Origins of Massive MIMO}

The concept behind the use of multiple antennas to achieve spatial directivity and diversity in wireless communications has been studied since the early $20^{\text {th }}$ century [47]. In the $1950 \mathrm{~s}$, the idea of combining the received signals from multiple antennas coherently, that is, adding them with their phases aligned, and letting the noise combine non-coherently, was shown to increase the SNR [48-50].

Towards the end of the $20^{\text {th }}$ century, further developments into finding the optimum linear combining of the received signals to maximize the SINR were achieved [51,52], followed by precoding methods that achieve similar goals when transmitting from an antenna array [53-57]. The spatial degrees of freedom offered by the antenna arrays were used to both increase the signal strength and reduce the interference between UEs operating on the same time-frequency resource. In particular, [52] is an early work in which 
multiplexing of UEs in the spatial domain is achieved by the use of several antennas at the BSs with an optimal linear combining strategy. Moreover, field trials with adaptive antenna arrays performed by Ericsson, Mannesmann, and AT\&T $[57,58]$ showed promising results to reduce interference and obtain lower BER compared to systems with fixed antenna patterns.

Between the late 1990s and early 2000s, the theoretical basis for the analysis of MIMO systems was introduced by [59-61], where the capacity of single-user MIMOs channel was characterized. In 2003, major breakthroughs were made in the field of information theory to describe the capacity of MU-MIMO systems [62-64]. These results formed the theoretical foundation for the gains that had earlier been demonstrated experimentally and by using less rigorous analysis. The MU-MIMO technology was proved to deliver higher sum capacity, but these benefits were counterbalanced by the need for complex signal processing techniques that relied on perfect CSI.

Despite the aforementioned advances, MU-MIMO was not adopted in $3 \mathrm{G}$, and it took almost ten years before the use of MU-MIMO took off in 4G. The fundamental reason might be the difficulty in acquiring CSI. When $4 \mathrm{G}$ was first designed, most cellular systems separated the UL and DL in the frequency domain through frequency-division duplex (FDD). To suppress interference by precoding in the DL, the BS needs to know the DL channels to its UEs very accurately. Methods based on estimating the direction of arrival (DoA) from UL signals and codebooks of beams from which the UEs report their preferred choice were considered. These methods could amplify the received signal power by an array gain, but did not provide sufficiently accurate CSI to suppress interference between UEs. One reason is that the channels at the typical cellular frequencies below $6 \mathrm{GHz}$ can generally not be parametrized in such a simple way, but contain many paths with widely different DoA. Another reason is that the number of BS antennas was small and roughly the same as the number of UEs. The practical gains were much lower than predicted by theory, which at this point did not provide a sufficient understanding of how to deal with imperfect CSI and therefore could not guide the implementation. Hence, the capacity of the 4G cellular systems was mainly improved by densification (deploying more BSs) and carrier aggregation to utilize more bandwidth.

In 2010, the use of a very large number of BS antennas in MU-MIMO systems, as compared to the number of spatially multiplexed UEs, was proposed in [65]. This paper is generally viewed as the origin of Massive MIMO, even if that terminology was not used in the paper. To acquire CSI for a large number of antennas, [65] proved that it is preferable to use TDD, where the UL and DL take place on the same frequencies but are separated in time, instead of FDD. It is then possible to learn all the necessary CSI from sending 
reference signals, known as pilots, in the UL only and then use it in both UL and DL. A number of pilots that is equal the number of multiplexed UEs is sufficient, irrespective of the number of BS antennas, and the impact of imperfect CSI is limited thanks to the very spatially directive signals that are obtained when using many antennas. Since 2010, the academia and industry have jointly developed a solid theory for Massive MIMO systems and its implementation $[7,8] .5 \mathrm{G}$ is the first generation of cellular communication systems that supports Massive MIMO [66]. As an example, the US operator Sprint has deployed hundreds of 5G Massive MIMO sites and reports $4 \times$ to $20 \times$ increase in sum data rates that has been added to the $4 \mathrm{G}$ network $[67,68]$.

\subsection{Communication Channel}

The communication channel is the input-output relationship between the transmitter and receiver. In wireless communications, electromagnetic waves are used to carry the information from the transmitter to the receiver. These waves propagate through the air encountering obstacles that reflect, diffract, and attenuate them. These phenomena change the amplitude and phase of the propagating signal, such that, in the far field, the received signal is given by a linear combination of attenuated and rotated versions of the transmitted signal that have taken different paths. Since the UEs and obstacles in a cellular network are mobile, the communication channel varies in time and frequency, which is referred to as a fading channel [69].

The radio frequencies that can be used for cellular wireless communications are set by governmental institutions. Thus, the signals need to be transmitted at a specific carrier frequency $f_{c}$ over a set bandwidth $B_{\mathrm{W}}$ as shown in Figure 2a. However, the signal processing is usually done at baseband (i.e., $f_{c}=0$ ) to simplify the implementation and then up/down converted to the desired carrier frequency. Since the physical signal that is transmitted is real valued, its spectrum is an even function (as shown in Figure 2a) and all the signal information is contained, in the bandwidth $B_{\mathrm{W}}$, either at positive or negative frequencies. Thus, the wireless channel, in the far field, can be described by a linear time varying system with a complex baseband representation (see Figure 2b) given by [69]

$$
y(t)=\sum_{i} a_{i}(t, f) x\left(t-\tau_{i}(t, f)\right)
$$

where $x(t)$ and $y(t)$ are the complex baseband transmitted and received signals at time $t$, respectively. The summation in (3) is done with respect to 


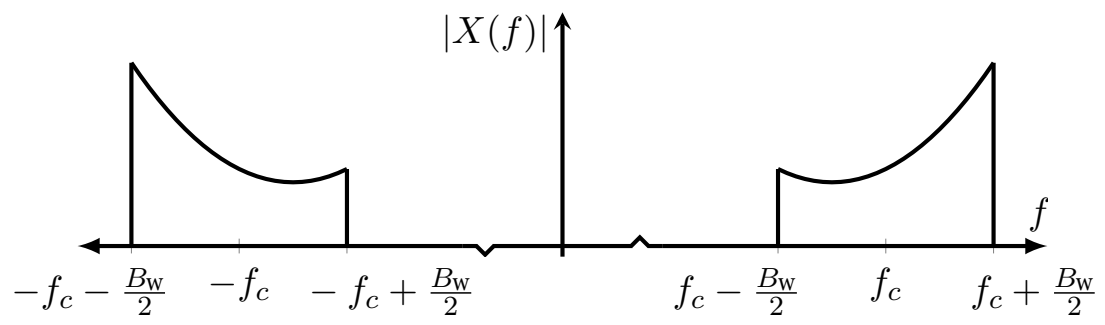

(a) Real-valued signal transmitted at a carrier frequency $f_{c}$.

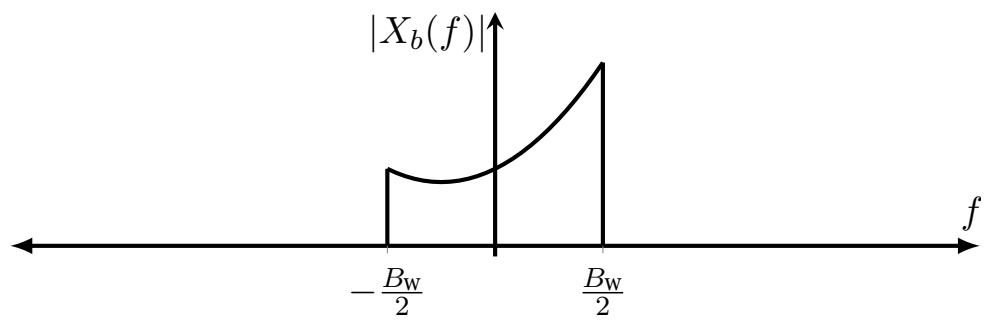

(b) Complex baseband equivalent signal.

Figure 2: Magnitude spectrum of passband and baseband signal.

the number of paths the transmitted signal takes to arrive to the receiver. The number of paths depends on the propagation conditions and the number of objects that can reflect, diffract or attenuated the signals. In scenarios with direct line-of-sight the number of paths can be only a few, whereas, in non-line-of-sight cases with many obstacles the number of paths can be in the hundreds or more. The attenuation and phase shift of the channel in the $i^{\text {th }}$ path is $a_{i}(t, f)$ and $\tau_{i}(t, f)$, respectively, and are functions of both time $t$ and frequency $f$. However, since the carrier frequency is usually much larger than the bandwidth, $f_{c} \gg B_{\mathrm{W}}$, the changes in $a_{i}(t, f)$ and $\tau_{i}(t, f)$ with respect to $f$, within $B_{\mathrm{W}}$, can be neglected. Thus, $a_{i}(t, f)=a_{i}(t)$ and $\tau_{i}(t, f)=\tau_{i}(t)$ are taken as the attenuation and phase shift of the channel in the $i^{t h}$ path at $f \approx f_{c}$. It is worth to stress that even though the effect of each path can be considered to be independent of frequency, the aggregated effect of the channel is indeed frequency dependent.

In general terms, the effect of the wireless channel can be classified in two parts:

Large-scale fading (LSF). This is caused by obstacles and movements that are orders of magnitude larger than the wavelength of the propagating signals. This effect is mainly responsible for attenuating the propagating signals due to pathloss, and shadowing from large ob- 


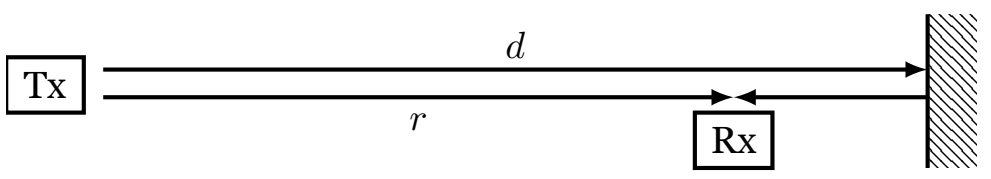

Figure 3: Two path propagation model. Tx and Rx stand for transmitter and receiver, respectively.

stacles. The LSF is considered to vary slowly in time (in the order of seconds or minutes depending on UE mobility) compared to the communication delay constraints (in the order of milliseconds). Moreover, since $B_{\mathrm{W}}$ is usually far lower than $f_{c}$, the attenuation changes from the LSF can be considered to be frequency independent.

Small-scale fading (SSF). This is caused by small obstacles and movements in the same order of magnitude as the wavelength of the propagating signals. This effect causes the signal to scatter, taking different paths, and resulting in phase shifts that may add constructively or destructively at the receiver, which is called multipath fading. The phase and amplitude changes occur in a fast time scale (in the order of milliseconds or less) and are frequency dependent due to the relative movements of the transmitter, receiver and obstacles.

The time and frequency variations of the SSF are orders of magnitude faster than the LSF, and for the design of communication systems, it is important to understand how these fast variations behave. To gain some insights into the physical meaning of the SSF effects, consider the scenario in Figure 3 where the transmitted signal is perfectly reflected by an infinite wall creating two paths for the received signal. For simplicity, assume that the difference in distance between the paths is small enough so that the attenuation is the same and the transmission power is normalized such that $a_{0}(t)=a_{1}(t)=1$. Let $x(t)=\cos (2 \pi f t)$, then the received signal is

$$
\begin{aligned}
y(t) & =\cos \left(2 \pi f\left(t-\tau_{1}\right)\right)-\cos \left(2 \pi f\left(t-\tau_{2}\right)\right) \\
& =\cos \left(2 \pi f\left(t-\frac{r}{c}\right)\right)-\cos \left(2 \pi f\left(t-\frac{2 d-r}{c}\right)\right)
\end{aligned}
$$

where $\tau_{1}=r / c$ and $\tau_{2}=(2 d-r) / c$ are the time delays of the signal from the first and second path, respectively. The waves travel at the speed of light, denoted by $c$, so that the phase shift at a distance $r$ is the travel time of the wave, that is $r / c$. Note that the minus sign for the second path is introduced by the reflection upon the wall [69]. 
The fast time variations are mainly due to the Doppler shifts introduced by relative movements of the transmitter, receiver, or obstacles. In the example shown in Figure 3, consider that the transmitter is fixed and the receiver is moving in the direction of propagation (towards the wall). The position of the receiver at time $t$ is given by $r=r_{0}+v t$ where $r_{0}$ is the initial position and $v>0$ is the constant velocity of the receiver, the received signal is then

$$
\begin{aligned}
y(t) & =\cos \left(2 \pi f\left(\left(1-\frac{v}{c}\right) t-\frac{r_{0}}{c}\right)\right)-\cos \left(2 \pi f\left(\left(1+\frac{v}{c}\right) t-\frac{2 d-r_{0}}{c}\right)\right) \\
& =2 \sin \left(2 \pi f\left(t-\frac{d}{c}\right)\right) \sin \left(2 \pi f\left(\frac{v t}{c}+\frac{r_{0}-d}{c}\right)\right) .
\end{aligned}
$$

The Doppler effect causes the received signals from the two paths to have frequency shifts of $D_{1}=-f v / c$ and $D_{2}=f v / c$, respectively. The difference between the Doppler shifts is called the Doppler spread and is given by $D_{s}=D_{2}-D_{1}$. The Doppler spread dictates the time period of the amplitude variations and for this example the period is $2 / D_{s}$, which corresponds to the time period of the second sinus in (5). In a more general scenario, where there are multiple paths with different Doppler shifts, the Doppler spread is defined as $D_{s}=\max _{i j}\left|D_{i}-D_{j}\right|$. In such cases, the aggregated effect of multiple Doppler shifts is often modeled as a realization of a random variable. The time interval between significant changes of the channel, in an order of magnitude sense, is called the coherence time and is inversely proportional to the Doppler spread. Thus, within this time period the channel can be approximated by a time-invariant system. A rule-of-thumb definition of the coherence time is given by [69]

$$
T_{c}=\frac{1}{4 D_{s}} .
$$

The variations in frequency are caused by the time difference between paths that the received signal has taken. In the example from Figure 3, the phase difference in the signal received from the two paths, shown in (4), is $2 \pi f T_{d}+\pi$ where $T_{d}=\tau_{2}-\tau_{1}$ is the time difference between the paths called the delay spread. By taking the Fourier transform of (4), the magnitude of the frequency response of the system can be computed as

$$
\left|\frac{Y(f)}{X(f)}\right|=\left|\mathrm{e}^{-j 2 \pi f \tau_{1}}-\mathrm{e}^{-j 2 \pi f \tau_{2}}\right|=2\left|\sin \left(\pi f T_{d}\right)\right|
$$

where the frequency period of these variations, that is the period of the sinusoid in (7) in terms of $f$, is $2 / T_{d}$. In a more general case where there are several paths with different delays, the delay spread can be defined as 
$T_{d}=\max _{i, j}\left|\tau_{i}-\tau_{j}\right|$. In this case, the aggregated effect of the delays introduced by many paths is often modeled by a random variable. The bandwidth interval in which the channel undergoes significant changes, in an order of magnitude sense, is called the coherence bandwidth and is inversely proportional to the delay spread. Thus, within this frequency period the frequency response of the channel can be considered to be approximately flat. A ruleof-thumb definition of the coherence bandwidth is given by [69]

$$
B_{c}=\frac{1}{2 T_{d}} .
$$

From the results in (6) and (8), if the received signal is observed during a coherence time and within a coherence bandwidth, the channel can be approximated by a linear time invariant system with flat frequency response. This time-frequency block is called the coherence block.

Based on the sampling theorem [70], a continuous time complex-valued signal with spectrum contained in a finite bandwidth within $\left[-B_{c} / 2, B_{c} / 2\right]$, can be represented by a discrete time complex-valued signal via pulse amplitude modulation. The discrete time complex-valued signal is composed of complex samples taken at the sampling rate of $f_{s} \geq B_{c}$. Then, a signal that is contained in a coherence time $T_{c}$ and a coherence bandwidth $B_{c}$ can be represented by $\tau_{c}=T_{c} B_{c}$ complex samples which constitute a coherence block. These complex samples are also referred to as the degrees of freedom or channel uses that are available in the coherence block. Finally, the discrete time complex baseband representation of (3) in a coherence block is

$$
y[n]=h \sqrt{\rho} x[n] \in \mathbb{C} \quad \text { for } \quad n \in\left\{1, \ldots, \tau_{c}\right\}
$$

where $x[n]$ and $y[n]$ are the discrete time transmitted and received signals, respectively, obtained by sampling $x(t)$ and $y(t)$ at a rate $f_{s}=B_{c}$. This is called the Nyquist sampling rate and it is going to be used throughout this dissertation. The aggregated effect of the channel is given by $h \in \mathbb{C}$. The term $\rho$ is the transmission energy per symbol and since the sampling frequency is the same as the bandwidth, the transmission power is $\rho B_{c}$.

\subsection{Properties of Massive MIMO Channels}

To explain how the channel properties affect the communication between the transmitter and receiver, consider a single-input multiple-output (SIMO) system where the receiver is equipped with $M$ antennas so that $\mathbf{h} \in \mathbb{C}^{M \times 1}$ corresponds to the communication channel. Assume for simplicity that the coherence block is infinite so that the channel can be considered to be 
deterministic and perfectly known by the transmitter and the receiver. Thus, the received signal in one arbitrary symbol of the coherence block is

$$
\mathbf{y}=\mathbf{h} \sqrt{\rho} x+\mathbf{n} \in \mathbb{C}^{M \times 1} .
$$

The transmitted information-bearing signal is represented by a zero-mean random variable $x \in \mathbb{C}$ such that $\mathbb{E}\left\{|x|^{2}\right\}=1$ and $\rho$ is the average transmitted energy per symbol. The receiver noise is independently distributed as $\mathbf{n} \sim \mathcal{C N}\left(\mathbf{0}, \sigma^{2} \mathbf{I}_{M}\right)$ with $\sigma^{2}$ corresponding to the average noise energy per symbol. The maximum achievable performance of this system is well defined by Shannon's capacity theorem $[7,8,69]$ and it is given by

$$
C_{\text {SIMO }}=\log _{2}\left(1+\frac{\rho\|\mathbf{h}\|^{2}}{\sigma^{2}}\right)[\mathrm{bpcu}] .
$$

The capacity indicates the maximum rate of information between the transmitter and receiver (here expressed in bits per channel use [bpcu]) that can be achieved with an arbitrarily low error probability (this is explained with more detail in Section 2.5). It can be seen from (11) that the capacity increases logarithmically with the channel gain, $\|\mathbf{h}\|^{2}$, which in turn increases with the number of antennas. ${ }^{1}$

Now consider the UL of a MU-MIMO system with two UEs denoted as $\mathrm{UE}_{1}$ and $\mathrm{UE}_{2}$. The $\mathrm{BS}$ is equipped with $M$ antennas so that $\mathbf{h}_{1}, \mathbf{h}_{2} \in \mathbb{C}^{M \times 1}$ correspond to the channels between the $\mathrm{BS}$ and $\mathrm{UE}_{1}, \mathrm{UE}_{2}$, respectively. As before, let the channels be deterministic and perfectly known. The received signal in one arbitrary symbol of the coherence block is

$$
\mathbf{y}=\mathbf{h}_{1} \sqrt{\rho} x_{1}+\mathbf{h}_{2} \sqrt{\rho} x_{2}+\mathbf{n} \in \mathbb{C}^{M \times 1} .
$$

The transmitted signals of $\mathrm{UE}_{1}$ and $\mathrm{UE}_{2}$ are the zero-mean random variables $x_{1}, x_{2} \in \mathbb{C}$ such that $\mathbb{E}\left\{\left|x_{1}\right|^{2}\right\}=1$ and $\mathbb{E}\left\{\left|x_{2}\right|^{2}\right\}=1$, respectively. In this case, the sum capacity is given by $[7,69]$

$$
\begin{aligned}
C_{\mathrm{SUM}} & =\log _{2}\left(\operatorname{det}\left(\mathbf{I}_{M}+\frac{\rho}{\sigma^{2}}\left(\mathbf{h}_{1} \mathbf{h}_{1}^{H}+\mathbf{h}_{2} \mathbf{h}_{2}^{H}\right)\right)\right)[\mathrm{bpcu}] \\
& =\log _{2}\left(\left(1+\frac{\rho}{\sigma^{2}}\left\|\mathbf{h}_{1}\right\|^{2}\right)\left(1+\frac{\rho}{\sigma^{2}}\left\|\mathbf{h}_{2}\right\|^{2}\right)-\frac{\rho^{2}}{\sigma^{4}}\left|\mathbf{h}_{1}^{H} \mathbf{h}_{2}\right|^{2}\right)[\mathrm{bpcu}]
\end{aligned}
$$

\footnotetext{
${ }^{1}$ This holds in cases where the aperture of the array increases proportionally with the number of antennas, that is, when $\|\mathbf{h}\|^{2}$ grows linearly with $M$. Note that this linear growth eventually breaks down due to the principle of energy conservation. However, it takes a very large number of antennas before it does, see [71] for details on the exact asymptotic behavior.
} 
and is maximized for channels that are orthogonal (i.e., $\mathbf{h}_{1}^{H} \mathbf{h}_{2}=0$ removes the negative term in (13)) which yields

$$
C_{\mathrm{SUM}}=\log _{2}\left(1+\frac{\rho}{\sigma^{2}}\left\|\mathbf{h}_{1}\right\|^{2}\right)+\log _{2}\left(1+\frac{\rho}{\sigma^{2}}\left\|\mathbf{h}_{2}\right\|^{2}\right)[\mathrm{bpcu}] .
$$

This result corresponds to the sum of the capacity in (11) of two individual SIMO systems. Therefore, a desirable property of MU-MIMO channels is

$$
\mathbf{h}_{i}^{H} \mathbf{h}_{j}= \begin{cases}0 & \text { for } i \neq j \\ \left\|\mathbf{h}_{i}\right\|^{2} & \text { for } i=j\end{cases}
$$

which is called favorable propagation.

\subsubsection{Stochastic Channel Model}

In cellular networks, the channel varies in time and frequency and the coherence block approximation would only be reasonable for a limited number of samples $\tau_{c}$ that are given by the propagation environment. Thus, the assumption of deterministic or perfectly known channels is dropped for the rest of this dissertation. As seen in Section 2.2, the LSF effect depends on the UE's mobility and usually changes slowly compared to the communication delay constraints, thus it is easy to track, and in this dissertation, is considered to be fixed and known. On the other hand, the SSF introduces variations in the amplitude and phase of transmitted signals that are in the same order, or faster than the communication delay constraints. Since the propagation environment changes continuously, it is hard to track each path that the transmitted signal takes to evaluate the channel deterministically. Thus, a stochastic model is used to gain insights into the performance of the system under fading channels.

As it is shown in Section 2.2, the communication channel within a coherence block contains the aggregated effect of the many different paths that signals travel from transmitter to receiver. Thus, motivated by the central limit theorem, the channel between $\mathrm{UE}_{i}$ and the $\mathrm{BS}$ is modeled as a random vector distributed as $\mathbf{h}_{i} \sim \mathcal{C N}\left(\mathbf{0}, \mathbf{R}_{i}\right)$ having independent realizations across different coherence blocks but remaining constant in each block. The effect of the SSF is comprised in the realizations of $\mathbf{h}_{i}$ and the LSF effect is given by the spatial correlation matrix $\mathbf{R}_{i}$ which is assumed to be fixed and known. This is called the block fading model with correlated Rayleigh fading and it is adopted throughout this dissertation.

This channel model provides a good approximation of different propagating environments ranging from rich scattering (i.e., $\mathbf{R}_{i}$ is a scaled identity 
matrix) to highly correlated channels (i.e., $\mathbf{R}_{i}$ has large eigenvalue variations). The former occurs when the signals between the UEs and the BS take many different paths and arrive in many different directions, whereas in the latter, the signals between the UEs and the BS are confined to a small range of spatial directions. More details on channel models for Massive MIMO systems can be found in [8, Ch. 7].

Note that in general, favorable propagation does not hold in real propagation environments. In particular, for Rayleigh fading channels, the inner product between two channel vectors is a random variable and favorable propagation does not appear in general. However, in Massive MIMO the main assumption is that the number of BS antennas $M$ is large, and in this case, two channel properties arise thanks to the law of large numbers:

Asymptotic favorable propagation. It occurs when the normalized inner products between channels from (or to) different UEs become asymptotically orthogonal as $M$ increases. That is for $i \neq j$

$$
\frac{\mathbf{h}_{i}^{H} \mathbf{h}_{j}}{\sqrt{\mathbb{E}\left\{\left\|\mathbf{h}_{i}\right\|^{2}\right\} \mathbb{E}\left\{\left\|\mathbf{h}_{j}\right\|^{2}\right\}}} \stackrel{\text { a. s. }}{\longrightarrow} 0 \quad \text { as } \quad M \rightarrow \infty
$$

where a. s. denotes almost sure convergence.

Channel hardening. It happens when the squared norm of the channel normalized by $M$ becomes deterministic as $M$ increases, that is,

$$
\frac{\left\|\mathbf{h}_{i}\right\|^{2}}{M}-\frac{1}{M} \operatorname{tr}\left(\mathbf{R}_{i}\right) \stackrel{\text { a. s. }}{\longrightarrow} 0 \quad \text { as } \quad M \rightarrow \infty,
$$

where $\mathbb{E}\left\{\left\|\mathbf{h}_{i}\right\|^{2}\right\}=\operatorname{tr}\left(\mathbf{R}_{i}\right)$.

These properties hold for a wide range of Massive MIMO channels, with different rates of convergence, and simplify its implementation $[7,8]$. In particular, for Rayleigh fading channels such as the ones considered in this dissertation, these properties hold under the following conditions: $i$ ) the average channel gain $\beta_{i}=\operatorname{tr}\left(\mathbf{R}_{i}\right) / M$ remains strictly positive as $M \rightarrow \infty$, which means that the aperture of the array grows with $M$ such that the channel gain $\left\|\mathbf{h}_{i}\right\|^{2}$ is proportional to $M$. ii) the spectral norm of $\mathbf{R}_{i}$ (i.e., largest eigen value) is bounded, meaning that the channel gain is spread among many directions and is not concentrated in a few strong ones as $M \rightarrow$ $\infty$. It is worth mentioning that these properties are based on asymptotic laws that eventually break down due to the law of energy conservation. However, it takes a very high number of antennas for these asymptotic laws to break 
down [71]. In addition, for practical number of BS antennas, these laws are observed in measured Massive MIMO channels [72].

Thanks to asymptotic favorable propagation, the channels between the BS and different UEs are easier to separate in the spatial domain as $M$ increases. In the case when linear signal processing is used to detect the data symbols, the asymptotic favorable propagation effect reduces the interference between UEs as $M$ grows large (as an example, see the last term inside the logarithm in (13)). On the other hand, channel hardening allows the power control to be independent from the SSF variations and it makes the channel estimation in the DL easier. Thus, these channel properties allow simple linear processing methods to perform well, providing high SE to several UEs simultaneously with low complexity. These effects are explained with further detail in Section 2.6.

\subsubsection{Impact of Spatial Correlation}

The channel between $\mathrm{UE}_{i}$ and the BS is defined by the vector $\mathbf{h}_{i} \in \mathbb{C}^{M}$ which can be described by its gain $\left\|\mathbf{h}_{i}\right\|^{2}$ and its direction $\mathbf{h}_{i} /\left\|\mathbf{h}_{i}\right\|$. Uncorrelated channels are defined by having independent gain and directions, in which, the latter are equally distributed in a $M$-dimensional sphere. All other channels that do not have these properties are correlated channels.

The spatial correlation matrix $\mathbf{R}_{i}$ describes how the channel gain is spread among spatial directions. In Massive MIMO, the interference between the spatially multiplexed UEs depends on the similarity between the spatial directions spanned by the spatial correlation matrices of UEs. To illustrate this concept, consider a two-user MU-MIMO system such as in (12) with $\rho=\sigma^{2}=1$ where the channels from $\mathrm{UE}_{1}$ and $\mathrm{UE}_{2}$ to the BS are distributed as $\mathbf{h}_{1} \sim \mathcal{C N}\left(\mathbf{0}, \mathbf{R}_{1}\right)$ and $\mathbf{h}_{2} \sim \mathcal{C N}\left(\mathbf{0}, \mathbf{R}_{2}\right)$, respectively. The eigenvalue decompositions of the spatial correlation matrices are given by $\mathbf{R}_{1}=\mathbf{U D}_{1} \mathbf{U}^{H}$ and $\mathbf{R}_{2}=\mathbf{U} \mathbf{D}_{2} \mathbf{U}^{H}$. Here, $\mathbf{U}$ is a unitary matrix (i.e., $\mathbf{U}^{H} \mathbf{U}=\mathbf{U} \mathbf{U}^{H}=\mathbf{I}_{M}$ ) containing the eigenvectors with all spatial directions. For some BS antenna array geometries, such as uniform linear arrays, the eigenvector matrix $\mathbf{U}$ can be approximated by a discrete Fourier transform matrix which is userindependent [73]. The eigenvalues are contained in the diagonal matrices $\mathbf{D}_{1}$ and $\mathbf{D}_{2}$ indicating how the channel gain is spread among the spatial 


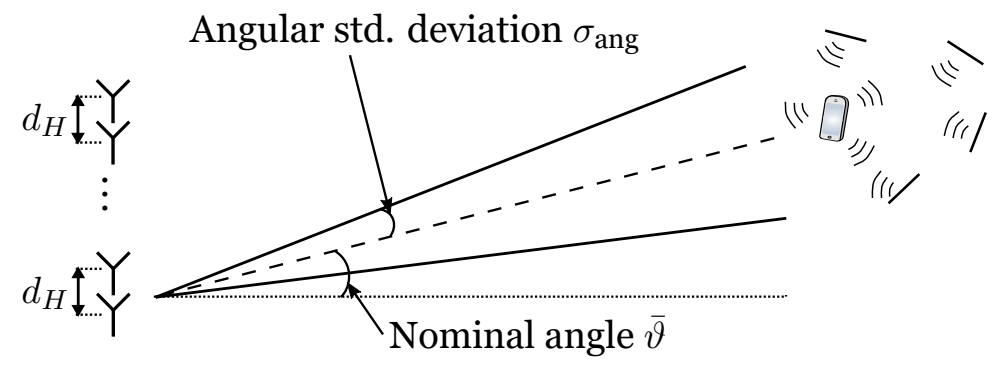

Figure 4: Local scattering model for spatially correlated channels.

directions. Consider the following two extreme cases:

$$
\begin{aligned}
& \text { Case 1: } \quad \mathbf{D}_{1}=\mathbf{D}_{2}=\left[\begin{array}{cc}
\mathbf{I}_{\frac{M}{2}} & \mathbf{0} \\
\mathbf{0} & \mathbf{0}
\end{array}\right] \\
& \text { Case 2: } \quad \mathbf{D}_{1}=\left[\begin{array}{cc}
\mathbf{I}_{\frac{M}{2}} & \mathbf{0} \\
\mathbf{0} & \mathbf{0}
\end{array}\right], \quad \mathbf{D}_{2}=\left[\begin{array}{cc}
\mathbf{0} & \mathbf{0} \\
\mathbf{0} & \mathbf{I}_{\frac{M}{2}}
\end{array}\right] \text {, }
\end{aligned}
$$

where the average channel gain of both channels in both cases is the same, that is, $\mathbb{E}\left\{\left\|\mathbf{h}_{i}\right\|^{2}\right\}=\operatorname{tr}\left(\mathbf{D}_{i}\right)=M / 2$ for $i \in\{1,2\}$. By projecting the received signal $y$ in (12) onto the span of the spatial correlation matrix of each UE, the equivalent systems are obtained:

$$
\begin{array}{ll}
\text { Case 1: } & \mathbf{R}_{1} \mathbf{y}=\mathbf{R}_{2} \mathbf{y}=\left[\begin{array}{c}
\mathbf{g}_{1} \\
\mathbf{0}
\end{array}\right] x_{1}+\left[\begin{array}{c}
\mathbf{g}_{2} \\
\mathbf{0}
\end{array}\right] x_{2}+\mathbf{U}\left[\begin{array}{c}
\overline{\mathbf{n}}_{1} \\
\mathbf{0}
\end{array}\right] \\
\text { Case 2: } & \left\{\begin{array}{l}
\mathbf{R}_{1} \mathbf{y}=\left[\begin{array}{c}
\mathbf{g}_{1} \\
\mathbf{0}
\end{array}\right] x_{1}+\mathbf{U}\left[\begin{array}{c}
\overline{\mathbf{n}}_{1} \\
\mathbf{0}
\end{array}\right] \\
\mathbf{R}_{2} \mathbf{y}=\left[\begin{array}{c}
\mathbf{0} \\
\mathbf{g}_{2}
\end{array}\right] x_{2}+\mathbf{U}\left[\begin{array}{c}
\mathbf{0} \\
\overline{\mathbf{n}}_{2}
\end{array}\right]
\end{array}\right.
\end{array}
$$

where $\mathbf{g}_{i} \sim \mathcal{C N}\left(\mathbf{0}, \mathbf{I}_{\frac{M}{2}}\right)$, and $\overline{\mathbf{n}}_{i} \sim \mathcal{C N}\left(\mathbf{0}, \mathbf{I}_{\frac{M}{2}}\right)$ for $i \in\{1,2\}$. In case 1 , the spatial correlation matrices are parallel which means that the projection yields another MU-MIMO system with two UEs. On the other hand, in case 2 the spatial correlation matrices are orthogonal and the result of the projection is two parallel SIMO systems. Thus, the impact of spatial correlation can be highly beneficial in MU-MIMO systems when the spatial directions that span the correlation matrices of UEs are substantially different. Note that the cases in (15) are just theoretical examples to illustrate the impact of spatial correlation matrices and do not appear in practice. 
In this dissertation, the local scattering spatial correlation model is used where the spatial correlation matrices are determined by azimuth angles from the BS array to the UEs [8, Ch. 2]. This model is based on the array response of a uniform linear array at the BS with the UEs located in the far field such that the correlation coefficients are given by

$$
\left[\mathbf{R}_{i}\right]_{m n}=\beta_{i} \int e^{2 \pi j d_{H}(m-n) \sin (\vartheta)} f_{\text {ang }}(\vartheta) d \vartheta .
$$

The distance between antennas is $d_{H}$ (measured in number of wavelengths) and the angle of arriving/departing signals is $\vartheta$ with probability density function $f_{\text {ang }}(\vartheta)$. This model considers the scattering sources to be in the surroundings of the UEs (but not at the BS), see Figure 4. As a result, the received signals at the BS from each UE are concentrate around a deterministic nominal angle $\bar{\vartheta}$ such that $\vartheta=\bar{\vartheta}+\delta$ where $\delta$ is modeled as random angular deviation. In this dissertation, the angular deviation is distributed as $\delta \sim \mathcal{N}\left(0, \sigma_{\text {ang }}^{2}\right)$ where $\sigma_{\text {ang }}$ is the angular standard deviation measured in degrees. For low values of $\sigma_{\text {ang }}$, the resulting channels are highly spatially correlated (i.e., few large eigenvalues) and as $\sigma_{\text {ang }}$ increases the channels become less correlated (i.e., less variations between eigenvalues). Note that uncorrelated Rayleigh fading channels with $\mathbf{R}_{i}=\beta_{i} \mathbf{I}_{M}$ can be obtained from (17) by assuming the sine of the angles to be uniformly distributed as $\sin (\vartheta) \sim \mathcal{U}(-1,1)$. More details about the local scattering model are found in $[8$, Ch. 2].

In this dissertation, the analysis conducted in Papers A, B, C, E and F assumes i.i.d. Rayleigh fading channels which are a special case of uncorrelated Rayleigh channels where each element in the channel vector $\mathbf{h}_{i}$ is independent and identically distributed. On the other hand, Papers D and G incorporate the effects of spatial correlation.

\subsection{Channel Estimation}

In Massive MIMO, the coherent signal processing requires each antenna to be controlled separately, and this can be performed in the analog or digital domain. In an analog implementation, only the phase of the signals at each BS antenna are controlled through analog circuits (e.g., phase shifters) and must be the same for the entire bandwidth. Whereas in the digital case, the coherent combination of signals is done via digital signal processing (DSP) such that each BS antenna element requires a digital circuit. This, allows for both the phase and amplitude of the signals in each BS antenna to be controlled and varied over the bandwidth. 
The communication channel and hardware introduce different phaseshifts and amplitude changes for the different antennas. Thus, to achieve coherent combination of signals, the combined effects of the channel and hardware need to be estimated in every coherence block and compensated for in the UL and DL processing. However, there is no need to use phasecalibrated arrays in Massive MIMO since the phase variations between the antennas can be treated as part of the communication channel.

The common method for channel estimation consists in sending known signals, called pilots, from the transmitter to the receiver who uses the pilot knowledge to estimate the channel. In this case, the number of symbols needed to estimate the channel is given by the number of transmit antennas, but is independent of the number of receive antennas and multiple receivers can utilize the same pilot. Since the channel changes with time and frequency, pilot signals need to be sent periodically throughout the whole bandwidth to obtain accurate channel estimates, creating an overhead that reduces the fraction of symbols used for data transmission. It is only the BS that critically needs to learn the channels in order to align the phases in the UL and DL. To make Massive MIMO systems scalable with respect to the number of BS antennas, it is desirable to transmit pilot symbols in the UL only. The challenge is then to learn the DL channel, but this is solved by operating in TDD because, in this case, the UL and DL channels are reciprocal and the channel estimates obtained in the UL can be used for DL transmission [74].

To illustrate the importance of exploiting channel reciprocity, consider a MU-MIMO system with $M$ antennas at the BS serving $K$ UEs. The channel is considered static during a finite number of symbols denoted as $\tau_{c}$. Figure 5 shows an example of TDD and FDD transmission protocols for Massive MIMO systems. ${ }^{2}$ In the TDD set up, the UL and DL are separated in time, and are both contained in the same coherence block. Thus, only $K$ symbols are needed for sending UL pilots which enable the BS to estimate the UL channels, and by reciprocity, these estimates can be used for precoding in the DL. Note that in the DL, the UEs can accurately estimate the precoded channel, which is a scalar, by means of blind methods like in [75] without the need of transmitting more pilots. In the FDD set up, the UL and DL are separated in frequency, and each of them occur in different coherence blocks. Thus, $K$ pilot symbols are sent in the UL and $M$ are sent in the DL. Then, the UEs take $M$ channel uses to feedback the channel estimate coefficients to the BS. ${ }^{3}$ Thus, the total number of pilot symbols per coherence block needed

\footnotetext{
${ }^{2}$ Note that Figure 5 illustrates the amount of pilot overhead needed for the TDD and FDD protocols. In practice, there are guard times and delays needed to synchronize the hardware computations.

${ }^{3}$ Each UE needs to send $M$ channel coefficients. Thus, to use only $M$ channel uses, it is
} 


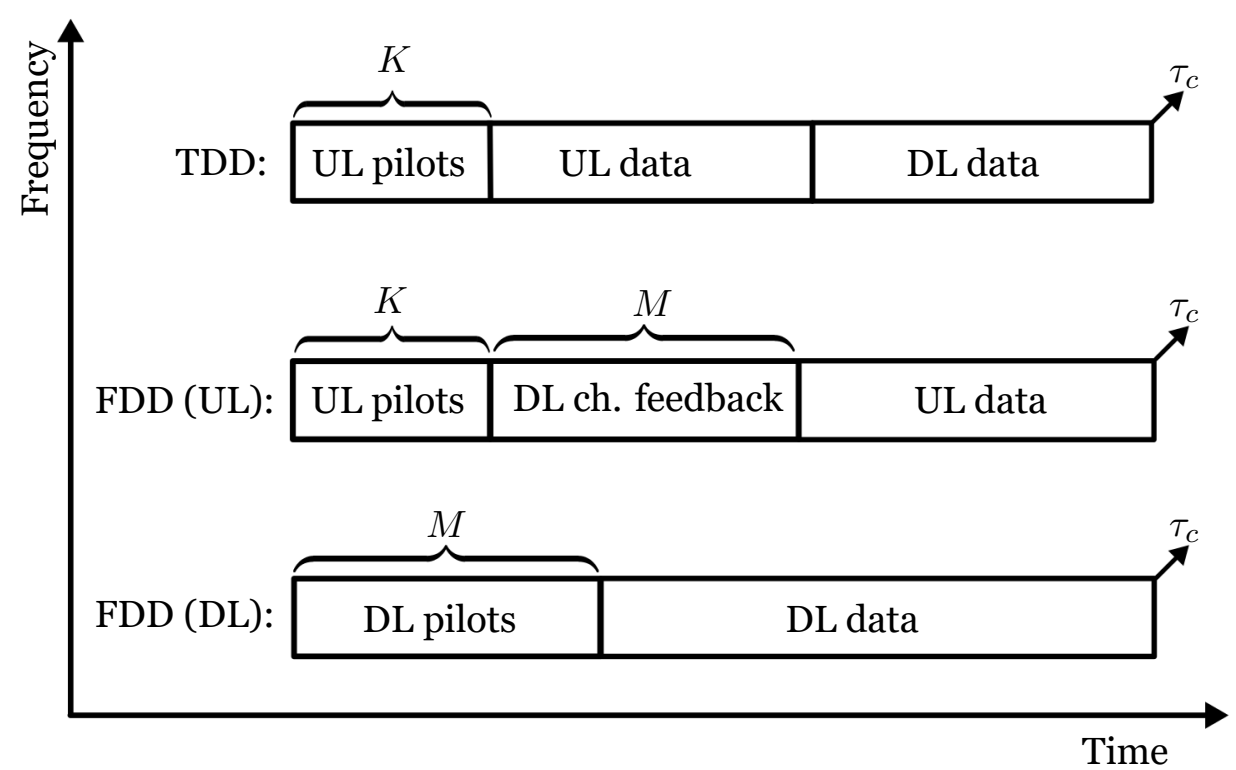

Figure 5: Transmission protocols for Massive MIMO systems.

with TDD is $K \leq \tau_{c}$, while with the average number of symbols per coherence block spent on channel estimation is $M+K / 2 \leq \tau_{c}$ in FDD. Note that these initial calculations do not consider any particular channel structure, which means that a reciprocity based TDD Massive MIMO system is scalable in terms of number of BS antennas, and can adapt to any channel structure.

In the case of FDD, depending on the channel structure, the estimation overhead can be significantly reduced, at the cost of lowering the spatial degrees of freedom available for spatial multiplexing. In a Massive MIMO $\mathrm{BS}$, the number of RF chains gives the spatial degrees of freedom and sets the complexity of implementation. To achieve the best performance, the Massive MIMO BS should have as many RF chains as BS antenna elements, however this may be costly and difficult to implement for very large arrays (e.g., beyond thousands of antennas). An approach to reduce the complexity of implementation and channel estimation overhead, is called hybrid precoding [76-78]. This method reduces the number of RF chains to a number comparable to the spatially multiplexed UEs and maps these to the antennas using analog circuitry, for example, a large number of analog phase shifters. Hybrid precoding is particularly attractive in millimeter wave implementations since a large number of antennas is needed to compensate for the fact

assumed that the feedback coefficients can be spatially multiplexed (i.e., $M \geq K$ ) which is the case in Massive MIMO systems. 


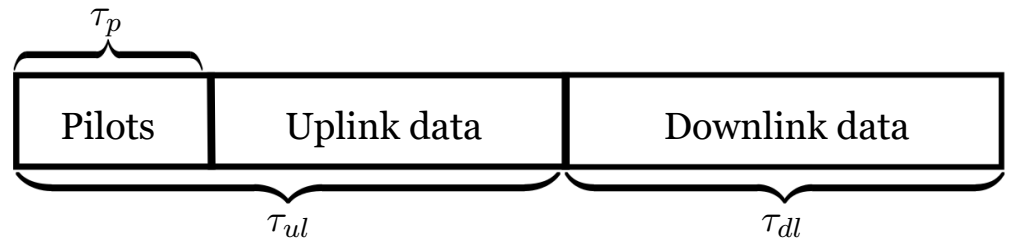

Figure 6: TDD transmission protocol of the baseline Massive MIMO system (also referred to as RP).

that the size of a fixed-gain antenna reduces with the wavelength, so many more are needed than in sub- $6 \mathrm{GHz}$ bands. The complexity of implementing many RF chains at millimeter wave frequencies is also higher due to the limited antenna size, so it will take time, and the combined efforts from academia and industry, before fully digital transceivers are commercially available. However, fully digital experimental setups already exists [79]. It is worth mentioning, that Massive MIMO as a technology is not bound to a specific range of carrier frequencies. The key feature in early deployments will likely be the array gain that, particularly, improves cell-edge conditions and is necessary at higher frequencies to compensate for the shrinking dimensions of fixed-gain antennas. As the number of UEs grows, the presence of channels with asymptotic favorable propagation will provide the largest SE gains by allowing for spatial multiplexing of many UEs with large antenna arrays at the BS. This can be implemented at any frequency.

\section{MMSE Channel Estimation}

In this dissertation, pilot based channel estimation is considered to conduct coherent signal processing. Pilot sequences are sent by the UEs in the UL from which the BS can estimate the channels. A TDD protocol is assumed and channel reciprocity within a coherence block is exploited so that the UL channel estimates can be used to process signals in the DL. Figure 6 illustrates a TDD communication protocol where $\tau_{u l}$ and $\tau_{d l}$ are the number of symbols in the coherence block used for UL and DL, respectively, such that $\tau_{c}=\tau_{u l}+\tau_{d l}$.

Consider the UL of a multicell MU-MIMO system where each BS deploys $M$ antennas and serves $K$ single-antenna UEs in its cell. Denote as $\mathrm{BS}_{l}$ the BS $l \in \Phi$, where $\Phi$ is a set with the indices of all BSs in the network, and $\mathrm{UE}_{l k}$ the UE $k$ that is served by $\mathrm{BS}_{l}$. The channel between the $M$ antennas of $\mathrm{BS}_{l}$ and $\mathrm{UE}_{l k}$ is defined as $\mathbf{h}_{l l k} \sim \mathcal{C N}\left(\mathbf{0}, \mathbf{R}_{l l k}\right)$, where $\mathbf{R}_{l l k}$ is the spatial 
correlation matrix which includes the LSF effects and $\beta_{l l k}=\operatorname{tr}\left(\mathbf{R}_{l l k}\right) / M$ is the average channel gain. As shown in Figure $6, \tau_{p}$ out of the $\tau_{u l}$ symbols in the UL are used to perform channel estimation. This corresponds to the baseline operation of Massive MIMO, which is referred to as RP. For ease of notation, the analysis is focused on $\mathrm{BS}_{0}$ and $\mathrm{UE}_{0 k} .{ }^{4}$ The pilot sequence used by $\mathrm{UE}_{0 k}$ is $\phi_{0 k} \in \mathbb{C}^{\tau_{p} \times 1}$ with elements having unit modulus, that is, $\left|\left[\phi_{0 k}\right]_{j}\right|=1 \forall j \in\left\{1, \ldots, \tau_{p}\right\}$. The pilots assigned to the UEs within the same cell are selected to be orthogonal, such that

$$
\phi_{0 k}^{H} \phi_{0 i}= \begin{cases}\tau_{p} & \text { for } k=i \\ 0 & \text { for } k \neq i\end{cases}
$$

This means that only $K \leq \tau_{p}$ UEs can be served simultaneously in one cell. Notice that only $\tau_{p}$ mutually orthogonal pilot sequences are available in the network, such that $\phi_{l i} \in \mathcal{V}_{\tau_{p}}$, where $\mathcal{V}_{\tau_{p}}$ is the set of all mutually orthogonal pilots in the system with cardinality $\tau_{p}$. Since the total number of UEs in all cells is usually larger than $\tau_{p}$, pilots need to be reused among cells. The set of indices containing the UEs that have the same pilot as $\mathrm{UE}_{0 k}$, including $\mathrm{UE}_{0 k}$, is defined as

$$
\mathcal{P}_{0 k}^{\mathrm{RP}}=\left\{(l, i): \phi_{0 k}^{H} \phi_{l i} \neq 0\right\} .
$$

The selection of the UEs that belong to this set depends on the pilot allocation scheme which is left undefined, for now, to obtain a more general result.

The received signal at the $M$ antennas of $\mathrm{BS}_{0}$ over the $\tau_{p}$ samples of the coherence block used for pilots is

$$
\mathbf{Z}_{0}=\sum_{l \in \Phi} \sum_{i=1}^{K} \mathbf{h}_{0 l i} \sqrt{q_{l i}^{\mathrm{RP}}} \boldsymbol{\phi}_{l i}^{T}+\mathbf{N}_{0} \in \mathbb{C}^{M \times \tau_{p}}
$$

where $\mathbf{N}_{0}$ is the noise matrix with elements independently distributed as $\left[\mathbf{N}_{0}\right]_{m j} \sim \mathcal{C N}\left(0, \sigma^{2}\right) \forall m \in\{1, \ldots, M\}, \forall j \in\left\{1, \ldots, \tau_{p}\right\}$, and with average noise energy per symbol $\sigma^{2}$. The transmission energy per pilot symbol sent by $\mathrm{UE}_{l i}$ is $q_{l i}^{\mathrm{RP}}$. The channel estimates are obtained by first performing a de-spreading operation on $\mathbf{Z}_{0}$ which yields the channel observation vector

$$
\mathbf{z}_{0 k}=\mathbf{Z}_{0} \frac{\phi_{0 k}^{*}}{\tau_{p} \sqrt{q_{0 k}^{\mathrm{RP}}}}=\sum_{(l, i) \in \mathcal{P}_{0 k}^{\mathrm{RP}}} \mathbf{h}_{0 l i} \sqrt{\frac{q_{l i}^{\mathrm{RP}}}{q_{0 k}^{\mathrm{RP}}}}+\frac{\overline{\mathbf{n}}_{0}}{\sqrt{\tau_{p} q_{0 k}^{\mathrm{RP}}}} \in \mathbb{C}^{M \times 1}
$$

where $\overline{\mathbf{n}}_{0} \sim \mathcal{C N}\left(\mathbf{0}, \mathbf{I}_{M} \sigma^{2}\right)$ is the projection of the noise matrix $\mathbf{N}_{0}$ onto $\phi_{0 k}^{*} /\left(\tau_{p} \sqrt{q_{0 k}^{\mathrm{RP}}}\right)$. Since the pilots are mutually orthogonal, no information

\footnotetext{
${ }^{4}$ Since the spatial distribution of BSs and UEs is kept unspecified, this notation does not influence the analysis.
} 
about the channel $\mathbf{h}_{00 k}$ is lost in the de-spreading operation. Second, the minimum MSE (MMSE) channel estimate of $\mathbf{h}_{00 k}$ is computed based on the observations $\mathbf{z}_{0 k}$. This result is summarized in the following lemma.

Lemma 1. The MMSE estimate of $\mathbf{h}_{00 k}$ based on $\mathbf{z}_{0 k}$ is

$$
\hat{\mathbf{h}}_{00 k}=\mathbf{R}_{00 k} \boldsymbol{\Psi}_{00 k}{ }^{-1} \mathbf{z}_{0 k}
$$

where

$$
\boldsymbol{\Psi}_{00 k}=\sum_{(l, i) \in \mathcal{P}_{0 k}^{\mathrm{RP}}} \mathbf{R}_{0 l i} \frac{q_{l i}^{\mathrm{RP}}}{q_{0 k}^{\mathrm{RP}}}+\frac{\sigma^{2}}{\tau_{p} q_{0 k}^{\mathrm{RP}}} \mathbf{I}_{M} .
$$

The estimation error is defined as $\tilde{\mathbf{h}}_{00 k}=\mathbf{h}_{00 k}-\hat{\mathbf{h}}_{00 k}$. The covariance matrices of the channel estimates and the estimation errors are

$$
\begin{aligned}
& \mathbb{E}\left\{\hat{\mathbf{h}}_{00 k} \hat{\mathbf{h}}_{00 k}^{H}\right\}=\mathbf{R}_{00 k} \mathbf{\Psi}_{00 k}{ }^{-1} \mathbf{R}_{00 k}, \\
& \mathbb{E}\left\{\tilde{\mathbf{h}}_{00 k} \tilde{\mathbf{h}}_{00 k}^{H}\right\}=\mathbf{R}_{00 k}-\mathbf{R}_{00 k} \mathbf{\Psi}_{00 k}{ }^{-1} \mathbf{R}_{00 k},
\end{aligned}
$$

respectively.

Proof. It follows from computing the mean of the channel $\mathbf{h}_{00 k}$ conditioned on the observations $\mathbf{z}_{0 k}$. This result is based on applying standard MMSE estimation techniques [80, Ch. 15.8$]$ to the problem at hand and it can be found in [8].

From this lemma, there are two key points the are important to mention:

\section{Estimation quality improves with longer pilots.}

The covariance matrix of the channel estimates depends on $\tau_{p}$ through $\boldsymbol{\Psi}_{00 k}$. To obtain ideal perfect channel estimation $\boldsymbol{\Psi}_{00 k} \rightarrow \mathbf{R}_{00 k}{ }^{5}$ (see (25)). In (23), it can be seen that as $\tau_{p}$ increases the effect of the noise vanishes. Moreover, as $\tau_{p}$ increases, there are more pilots available which means that they can be reused further apart in the network. This decreases the number of UEs in the set $\mathcal{P}_{0 k}^{\mathrm{RP}}$, reducing the pilot interference (see (23)). In the extreme case when $\tau_{p}$ is so large that all UEs in the network can have an orthogonal pilot sequence, and the normalized noise power $\sigma^{2} /\left(q_{0 k}^{\mathrm{RP}} \tau_{p}\right)$ is negligible compared to $\operatorname{diag}\left(\mathbf{R}_{00 k}\right)$, it follows that $\boldsymbol{\Psi}_{00 k} \rightarrow \mathbf{R}_{00 k}$. However, in practice $\tau_{p}$ is limited by the coherence block size which is set by the propagation environment.

\footnotetext{
${ }^{5}$ Te notation $\boldsymbol{\Psi}_{00 k} \rightarrow \mathbf{R}_{00 k}$ means that given the eigen value decomposition (EVD) $\mathbf{R}_{00 k}=$ UDU ${ }^{H}$, the matrix $\Psi_{00 k}$ would have the same eigen vectors $\mathbf{U}$ and the eigenvalues would tend to $\mathbf{D}$.
} 


\section{Channel estimates and errors are statistically independent under Gaussian observations.}

For MMSE estimation, the estimates are uncorrelated with the estimation errors by design. Since the channel observations $\mathbf{z}_{0 k}$ in (21) are Gaussian distributed, the MMSE estimates are linear in the observations which means that they coincide with the linear MMSE (LMMSE) estimates. In addition, the estimates and errors are jointly Gaussian random variables that are uncorrelated, and therefore, they are also statistically independent. This fact is commonly used in literature to calculated closed form expressions of lower bounds on the capacity $[7,8]$. However, notice that in cases where the observations are not Gaussian the estimates and errors remain uncorrelated but are not statistically independent. This fact plays an important role in the performance evaluation of Massive MIMO with SP, and under hardware distortions. This is explained with more detail in Chapter 3.

\subsection{Capacity Analysis}

To evaluate the performance of a communication system, a key metric is the maximum rate of information that can be conveyed from the transmitter to the receiver in a reliable way. An achievable rate is defined as an information rate (here measured in [bpcu]) that can be achieved by the communication between a transmitter and a receiver with an error probability that can be made arbitrarily low as the block-length of the coded information increases $[7,8,69]$. The capacity is defined as the maximum achievable rate that is reached when the block-length of the coded information goes to infinity. In other words, the capacity gives the best information rate that can be achieved error-free.

The ergodic capacity of a single cell MU-MIMO system under a fast fading channel and white Gaussian noise is known for the case where the receiver has perfect channel knowledge [81]. ${ }^{6}$ However, when the receiver does not known the channel or there is non-Gaussian interference, the capacity is unknown. In these cases, there are expressions for upper and lower bounds on the capacity that can serve as performance metrics. The SE analysis in dissertation is based on lower bounds on the capacity.

\footnotetext{
${ }^{6}$ The term ergodic capacity indicates that as the block-length of the coded information goes to infinity, all possible realizations of the channel (according to the channel distribution), will be observed. In the context of the block fading channel model, ergodicity means that the statistical properties of the channel do not vary over time or frequency. Thus, the capacity is achieved by encoding information over infinitely many coherence blocks.
} 
Recall that from the sampling theorem, a signal contained within a bandwidth $B_{\mathrm{W}}[\mathrm{Hz}]$ and a time $T_{c}$ [s], can be represented by $B_{\mathrm{W}} T_{c}$ complex samples. Thus, if $B_{\mathrm{W}}$ complex samples per second are used for communicating information within a bandwidth $B_{\mathrm{W}}$, an achievable rate of $\mathrm{R}$ [bpcu] is equivalent to an SE of R [bit/s/Hz]. For the MU-MIMO system model introduced in Section 2.4, the received data signal at $\mathrm{BS}_{0}$ in an arbitrary symbol of the coherence block is

$$
\mathbf{y}_{0}=\sum_{l \in \Phi} \sum_{i=1}^{K} \mathbf{h}_{0 l i} \sqrt{\rho_{l i}^{\mathrm{RP}}} s_{l i}+\mathbf{n}_{0} \in \mathbb{C}^{M \times 1}
$$

where $\mathbf{n}_{0} \sim \mathcal{C N}\left(\mathbf{0}, \mathbf{I}_{M} \sigma^{2}\right)$ is the noise vector with $\sigma^{2}$ as the average noise energy per symbol and $\rho_{l i}^{\mathrm{RP}}$ as the average transmission energy per data symbol from $\mathrm{UE}_{l i}$. The data symbol transmitted by $\mathrm{UE}_{l i}$ is $s_{l i}$ and it is assumed to be zero-mean and have unit variance. To perform coherent detection, linear signal processing is typically considered in Massive MIMO since it offers linear computational complexity in $M$. Thus, $\mathrm{BS}_{0}$ multiplies the received signal with the combining matrix $\mathbf{A}=\left[\mathbf{a}_{1}, \ldots, \mathbf{a}_{K}\right] \in \mathbb{C}^{M \times K}$ to obtain $K$ equivalent single-input single-output (SISO) systems corresponding to the $K$ multiplexed UEs. For $\mathrm{UE}_{0 k}$ the equivalent SISO received signal is then used as an estimate of the transmitted data symbol which is given by

$$
\begin{aligned}
\hat{s}_{0 k} & =\mathbf{a}_{k}^{H} \mathbf{y}_{0} \\
= & \underbrace{\mathbf{a}_{k}^{H} \mathbf{h}_{00 k} \sqrt{\rho_{0 k}^{\mathrm{RP}}} s_{0 k}}_{\text {Desired signal }}+\underbrace{\sum_{\substack{i=1 \\
i \neq k}}^{K} \mathbf{a}_{k}^{H} \mathbf{h}_{00 i} \sqrt{\rho_{0 i}^{\mathrm{RP}}} s_{0 i}}_{\text {Intracell interference }}+\underbrace{\sum_{l \in \Phi \backslash 0} \sum_{i=1}^{K} \mathbf{a}_{k}^{H} \mathbf{h}_{0 l i} \sqrt{\rho_{l i}^{\mathrm{RP}}} s_{l i}}_{\text {Intercell interference }}+\underbrace{\mathbf{a}_{k}^{H} \mathbf{n}_{0}}_{\text {Noise }} .
\end{aligned}
$$

To combine the received signals from each BS antenna coherently, the selection of $\mathbf{a}_{k}$ is based on the channel estimates that were obtained in Section 2.4. The capacity of the equivalent SISO system in (27) is unknown since the channels are unknown and the interference is non-Gaussian. A standard lower bound on the capacity can be obtained by applying the so called useand-then-forget technique [7], in which the channel estimates are used for constructing $\mathbf{a}_{k}$ but are then neglected when computing the capacity bound. Thus, the data estimates can be rewritten as

$$
\hat{s}_{0 k}=\underbrace{\mathbb{E}\left\{\hat{s}_{0 k} s_{0 k}^{*}\right\}}_{\begin{array}{c}
\text { Deterministic } \\
\text { effective channel }
\end{array}} s_{0 k}+\underbrace{\hat{s}_{0 k}-\mathbb{E}\left\{\hat{s}_{0 k} s_{0 k}^{*}\right\} s_{0 k}}_{\begin{array}{c}
\text { Non-Gaussian } \\
\text { effective noise }
\end{array}}
$$


and a lower bound on the capacity is given by $[7,8]$

$$
\mathbf{R}_{0 k}=\left(1-\frac{\tau_{p}}{\tau_{u l}}\right) \log _{2}\left(1+\operatorname{SINR}_{0 k}\right),
$$

where

$$
\begin{aligned}
\operatorname{SINR}_{0 k} & =\frac{\left|\mathbb{E}\left\{\hat{s}_{0 k} s_{0 k}^{*}\right\}\right|^{2}}{\operatorname{Var}\left\{\hat{s}_{0 k}-\mathbb{E}\left\{\hat{s}_{0 k} s_{0 k}^{*}\right\} s_{0 k}\right\}}, \\
& =\frac{\left|\mathbb{E}\left\{\mathbf{a}_{k}^{H} \mathbf{h}_{00 k}\right\}\right|^{2} \rho_{0 k}^{\mathrm{RP}}}{\operatorname{Var}\left\{\mathbf{a}_{k}^{H} \mathbf{h}_{00 k}\right\} \rho_{0 k}^{\mathrm{RP}}+\sum_{\substack{l \in \Phi \\
(l, i) \neq(0, k)}} \sum_{i=1}^{K} \mathbb{E}\left\{\left|\mathbf{a}_{k}^{H} \mathbf{h}_{0 l i}\right|^{2}\right\} \rho_{l i}^{\mathrm{RP}}+\mathbb{E}\left\{\left|\mathbf{a}_{k}^{H} \mathbf{n}_{0}\right|^{2}\right\}},
\end{aligned}
$$

where the pre-log factor in (29) corresponds to the overhead of using $\tau_{p}$ samples for channel estimation. ${ }^{7}$ The result in (30) holds for any SISO system with a deterministic effective channel and non-Gaussian effective noise as shown in (28). The effective SINR in (31) applies for linear processing and it holds for any choice of $\mathbf{a}_{k}$ or distribution of the channels $\mathbf{h}_{0 l i}$. The performance given by this bound may underestimate the real performance that can be achieved since the information available from the channel estimates is neglected when computing the bound itself. However, thanks to the effect of channel hardening, this bound tightens as $M$ grows large, which makes it a suitable metric to evaluate the performance of Massive MIMO [7]. In addition, this bound allows for a tractable analysis that results in closed-form rate expressions that provide key insights into the system performance. There are other bounds that are closer to the capacity and can be used to evaluate other scenarios where, for instance, channel hardening does not hold [82].

To select the combining matrix, some standard choices [8] are

$$
\mathbf{A}= \begin{cases}\hat{\mathbf{H}}_{00} & \mathrm{MR} \\ \hat{\mathbf{H}}_{00}\left(\hat{\mathbf{H}}_{00}^{H} \hat{\mathbf{H}}_{00}\right)^{-1} & \mathrm{ZF} \\ \left(\sum_{l \in \Phi} \sum_{i=1}^{K} \rho_{l i}^{\mathrm{RP}}\left(\hat{\mathbf{h}}_{0 l i} \hat{\mathbf{h}}_{0 l i}^{H}+\left(\mathbf{I}_{M}-\mathbf{R}_{0 l i} \mathbf{\Psi}_{0 l i}^{-1}\right) \mathbf{R}_{0 l i}\right)+\sigma^{2} \mathbf{I}_{M}\right)^{-1} \hat{\mathbf{H}}_{00} \mathbf{D}_{\rho_{0}^{\mathrm{RP}}} & \text { mMMSE }\end{cases}
$$

where $\hat{\mathbf{H}}_{00}=\left[\hat{\mathbf{h}}_{001}, \ldots, \hat{\mathbf{h}}_{00 K}\right]$ and $\mathbf{D}_{\rho_{0}^{\mathrm{RP}}}=\operatorname{diag}\left(\rho_{01}^{\mathrm{RP}}, \ldots, \rho_{0 K}^{\mathrm{RP}}\right)$.

The first choice in (32) is called maximum ratio (MR) combining, and it is the main signal processing technique used throughout this dissertation. The

\footnotetext{
${ }^{7}$ The result in (29) corresponds to the UL, so that $\tau_{c}=\tau_{u l}$. A similar approach can be used for computing a lower bound on the DL capacity, as done in $[7,8]$.
} 
idea behind MR combining is to maximize the desired signal (the first term in (27)). Thus, in cases where the noise is the main source of SE degradation and inter-user interference is low, such as low SNR scenarios, MR combining is a good choice. In addition, it has linear computational complexity in $M$ and $K$, and it can be implemented with a distributed architecture. The second choice is zero forcing (ZF), where the idea is to cancel the intracell interference (see the second term in (27)) that is introduced by other UEs in the same cell as $\mathrm{UE}_{0 k}$. This is a good choice in cases with high SNR where the interference effect is strong. The third choice is the multicell MMSE (mMMSE) and is the optimal linear processing where a balance between interference suppression and desired signal amplification is achieved taking into account the effects of intercell interference.

Note that mMMSE has always best performance, and ZF performs better than MR combining in high SNR scenarios, however mMMSE and ZF also have higher computational complexity due to the matrix inversion needed (see (32)). In multicell scenarios with uncorrelated Rayleigh fading channels and low SNR, the gap between mMMSE and MR combining becomes small as $M$ increases (see Section 2.6). On the other hand, in correlated Rayleigh fading channels the benefits of interference suppression are more pronounced. In particular, when the spatial correlation matrices are linearly independent, mMMSE overcomes the pilot contamination effect and achieves unlimited SE as $M$ grows to infinity [8, Ch. 4].

It is worth mentioning that, in scenarios where the channel estimates are non-Gaussian (as the ones studied in this dissertation) the analysis with $\mathrm{ZF}$ and mMMSE tends to be analytically intractable. Therefore, in this dissertation, the analysis is focused on MR combining where closed-form expressions are computed and clear insights into the phenomena that affect the performance can be obtained.

\subsection{Performance of Massive MIMO}

To evaluate the performance of Massive MIMO, the following three main performance metrics are used throughout this dissertation: SE, power consumption and EE. The SE is obtained from the capacity analysis depicted in Section 2.5. The general power consumption model used in this dissertation is based on [83], such that the power consumption per cell is

$$
\mathrm{P}_{\mathrm{CELL}}=\mathrm{P}_{\mathrm{FIX}}+\mathrm{P}_{\mathrm{BP}}+\mathrm{P}_{\mathrm{TX}-\mathrm{RX}}+\mathrm{P}_{\mathrm{RF}}+\mathrm{P}_{\mathrm{CD}}\left(B_{\mathrm{W}} \mathrm{SE}_{\mathrm{CELL}}\right)
$$

where $\mathrm{P}_{\mathrm{FIX}}$ is the fixed power consumption (e.g., site cooling), $\mathrm{P}_{\mathrm{BP}}$ accounts for the power consumed by baseband processing, $\mathrm{P}_{\mathrm{TX}-\mathrm{RX}}$ represents the power 
consumption of the circuitry in transceiver chains, $\mathrm{P}_{\mathrm{RF}}$ accounts for the $\mathrm{RF}$ transmission power, and $\mathrm{P}_{\mathrm{CD}}$ is the power consumption for coding, decoding, and backhaul that depends on the data rate per cell. The SE per cell is defined as $\mathrm{SE}_{\mathrm{CELL}}$ so that the data rate per cell is given by $B_{\mathrm{W}} \mathrm{SE}_{\mathrm{CELL}}[\mathrm{bit} / \mathrm{s}$ ] when sampling at the Nyquist rate. Finally, the EE is defined as

$$
\mathrm{EE}=\frac{B_{\mathrm{W}} \mathrm{SE}_{\mathrm{CELL}}}{\mathrm{P}_{\mathrm{CELL}}}
$$

The model in (33) includes the main sources of power consumption, and depending on the focus of the analysis, some terms might be lumped together into fixed terms or further expanded by using more detailed models.

The power consumption of baseband processing can be further expanded as

$$
\mathrm{P}_{\mathrm{BP}}=\mathrm{P}_{\mathrm{CE}}+\mathrm{P}_{\mathrm{LP}}
$$

where $\mathrm{P}_{\mathrm{CE}}$ is the power consumed by channel estimation processing and $\mathrm{P}_{\mathrm{LP}}$ is the power spent on linear signal processing. These terms are computed by counting the number of multiplications needed to perform channel estimation and linear processing per time unit (measured in flops, i.e., floating point operations per second), and dividing them by the computational efficiency of the hardware (measured in [flops/W]). The power consumption for baseband processing depends on the channel model assumptions and the type of processing used. For example, by assuming MR combining under an i.i.d. Rayleigh fading channel, the power consumption of baseband processing can be approximated as

$$
\mathrm{P}_{\mathrm{BP}}=\frac{B_{\mathrm{W}}}{L} 2 M K
$$

where $L$ is the computational efficiency of the hardware at the BS, measured in [flops/W]. If the channel is assumed to be spatially correlated, then one extra $M \times M$ matrix inversion and $K$ matrix multiplications (of size $M \times M$ ) need to done on each coherence block (see (22)). In addition, if interference suppression techniques are used for the linear processing, another matrix inversion operation ( $M \times M$ or $K \times K$ depending on the signal processing used) and a $M \times K$ matrix multiplication need to be added in each coherence block (see (32)). Thus, by counting the number of multiplications needed to perform the aforementioned matrix operations, the power consumption of baseband processing can be represented as a polynomial function of $M$ and $K$. The impact of computational efficiency is not the focus of this dissertation, therefore, it is included in the numerical analysis but the details on how to construct the power consumption of baseband processing is deferred to [8, Ch. 5]. In Massive MIMO, $M$ and $K$ are much larger than one which 
means that the number of baseband operations can be very high (e.g., tens or hundreds of Gflops). However, the development of microprocessor enables very high computational efficiency (e.g., in the order of tens [Gflops/W]) such that the matrix operations involved in baseband processing lead to reasonable power consumption (e.g., between a few [W] up to tens of [W]).

To analyze the impact of power control in the UL and power allocation in the DL, on the power consumption, the RF transmission power model can be expanded as

$$
\mathrm{P}_{\mathrm{RF}}=\underbrace{\frac{\tau_{p}}{\tau_{c}} \frac{B_{\mathrm{W}}}{\eta} \sum_{k^{\prime}=1}^{K} q_{k}}_{\text {UL transmission pilot power }}+\underbrace{\left(\frac{\tau_{u l}-\tau_{p}}{\tau_{c}}\right) \frac{B_{\mathrm{W}}}{\eta} \sum_{k^{\prime}=1}^{K} \rho_{k}}_{\text {UL transmission data power }}+\underbrace{\left(\frac{\tau_{d l}}{\tau_{c}}\right) \frac{B_{\mathrm{W}}}{\eta} \sum_{k^{\prime}=1}^{K} p_{k}}_{\text {DL transmission data power }}
$$

to account for the influence of selecting the UL pilot transmission energy per symbol, or UL/DL average transmission energy per symbol. The term $\eta$ is the efficiency of the power amplifier and $p_{k}$ is the DL transmission energy per symbol allocated to $\mathrm{UE}_{k}$.

In the analysis of hardware quality, the impact of the ADC bit resolution in the power consumption is highly important. Thus, the power consumption of transceiver chains can be decomposed as follows

$$
\mathrm{P}_{\mathrm{TX}-\mathrm{RX}}=K \mathrm{P}_{\mathrm{UE}}+M\left(\mathrm{D}_{0}+2 \mathrm{P}_{\mathrm{ADC}}\right)
$$

where $\mathrm{P}_{\mathrm{UE}}$ is the power consumed by the circuitry at each $\mathrm{UE}, \mathrm{D}_{0}$ includes the power consumption per RF chain that is independent of the ADC bit resolution and

$$
\mathrm{P}_{\mathrm{ADC}}=\mathrm{D}_{1} 2^{\varphi b}
$$

is the power consumed by the ADC. ${ }^{8}$ The term $b$ corresponds to the ADC bit resolution and $D_{1} \geq 0$ and $\varphi \geq 0$ are set constants. Typical values are $\mathrm{D}_{1} \in\left[10^{-5}, 10^{-2}\right]\left[\mathrm{W} /\right.$ conversion step], $\varphi \in[1,2] .^{9}$ The model in (39) is chosen to encompass different architectures and implementations of ADCs found in the literature $[39,84-86] .{ }^{10}$

To illustrate the key benefits and characteristics of Massive MIMO, the UL MU-MIMO model presented in Section 2.5 is used. The communication

\footnotetext{
${ }^{8}$ The factor of two multiplying $\mathrm{P}_{\mathrm{ADC}}$ in (38) accounts for the two ADCs needed for the in-phase and quadrature branches in each RF chain.

${ }^{9}$ The choice of $\mathrm{D}_{1}$ and $\varphi$ can be related to the figure-of-merit (FoM) of ADCs where small values of $D_{1}$ are paired with larger values of $\varphi$ and vice versa [84].

${ }^{10}$ It is worth mentioning that in Papers $\mathrm{E}$ and $\mathrm{F}$ the value of $\mathrm{D}_{1}$ was taken based on the state of the art ADC research [84] which may not yet be commercially available. Thus, in Paper G the parameter $D_{1}$ has been chosen to have more a conservative value that matches available off-the-shelf ADCs.
} 
channels are modeled as i.i.d. Rayleigh fading to simplify the analysis and readability. However, all Massive MIMO features presented in the following analysis extend to correlated channels.

The SE of $\mathrm{UE}_{0 k}$ with MR combining base on the lower bound on the capacity in (29) is given by $[7,8]$

$$
\mathrm{SE}_{0 k}=\left(\frac{\tau_{u l}-\tau_{p}}{\tau_{c}}\right) \log _{2}\left(1+\frac{M \beta_{00 k} \rho_{0 k}^{\mathrm{RP}}}{\sum_{(l, i) \in \mathcal{P}_{0 k}^{\mathrm{RP}} \backslash(0, k)} \frac{\beta_{0 l i}^{2} q_{l i}^{\mathrm{RP}} \rho_{l i}^{\mathrm{RP}}}{\beta_{00 k} q_{0 k}^{R P}}+\frac{1}{\gamma_{0 k}}\left(\sum_{l \in \Phi} \sum_{i=1}^{K} \beta_{0 l i} \rho_{l i}^{\mathrm{RP}}+\sigma^{2}\right)}\right)
$$

where

$$
\gamma_{0 k}=\frac{q_{0 k}^{\mathrm{RP}} \tau_{p} \beta_{00 k}}{\sum_{(l, i) \in \mathcal{P}_{0 k}^{\mathrm{RP}}} q_{l i}^{\mathrm{RP}} \tau_{p} \beta_{0 l i}+\sigma^{2}} .
$$

Note that for i.i.d. Rayleigh fading channels $\mathbf{R}_{00 k} \mathbf{\Psi}_{00 k}{ }^{-1}=\gamma_{0 k} \mathbf{I}_{M}$. Thus, $\gamma_{0 k} \rightarrow 0$ means poor channel estimation quality, whereas $\gamma_{0 k} \rightarrow 1$ means perfect channel estimation.

The numerator of the SINR in (40) is referred to as the coherent gain and it scales with $M$, the first term in the denominator is called the coherent interference and the second term is the non-coherent interference. In this dissertation, coherent interference refers to the interference that combines constructively in the detection process, because it comes from the same spatial direction as the channel estimates, and therefore in i.i.d. Rayleigh fading channels it scales with $M$. Instead, non-coherent interference refers to interference that combines in a non-constructive way and does not scale with $M$.

The closed form expression in (40) allows for a simple evaluation of the $\mathrm{SE}$ and shows key insights into the performance of Massive MIMO. In what follows, some numerical results are also introduced to exemplify some of the main properties of Massive MIMO. Three cases of study for the numerical results are introduced which correspond to Figures 7-9. To deliver the main insights in a concise manner, first the main assumptions on each case of study are introduced, and second, a point-by-point explanation of the key characteristics of Massive MIMO is presented with references to previous analysis and said numerical results.

Case 1) Multicell MU-MIMO system: Figure 7 shows the SE per cell for a multicell MU-MIMO system with squared cells of side length $R_{c}[\mathrm{~m}]$ where $K$ UEs are distributed uniformly within each cell with a minimum distance to 
Table 1: Parameters for numerical analysis.

\begin{tabular}{c|c|c} 
Parameter & Symbol & Value \\
\hline Fixed propagation loss at 1 [m] & $\omega$ & $35[\mathrm{~dB}]$ \\
Cell radius & $R_{c}$ & $200[\mathrm{~m}]$ \\
Pathloss exponent & $\alpha$ & 3.76 \\
System bandwidth & $\mathrm{B}_{\mathrm{W}}$ & $20[\mathrm{MHz}]$ \\
Const. related to ADC saturation level & $\zeta$ & 1.6 \\
Consts. related to the ADC FoM & $\mathrm{D}_{1}$ & $0.006[\mathrm{~W} /$ conv-step $]$ \\
& $\varphi$ & 1 \\
Fixed power consumption & $\mathrm{P}_{\mathrm{FIX}}$ & $10[\mathrm{~W}]$ \\
Circuit power per active UE & $\mathrm{P}_{\mathrm{UE}}$ & $0.1[\mathrm{~W}]$ \\
Circuit power per BS antenna (ind. of $b$ ) & $\mathrm{D}_{0}$ & $0.1[\mathrm{~W}]$ \\
Power coding/decoding & $\mathrm{P}_{\mathrm{CD}} B_{\mathrm{W}}$ & $0.023[\mathrm{~W} / \mathrm{bit}]$ \\
Computational efficiency & $L$ & $12.8[\mathrm{Gflops} / \mathrm{W}]$ \\
Noise power & $\sigma^{2} B_{\mathrm{W}}$ & $2 \cdot 10^{-13}[\mathrm{~W}]$ \\
\hline
\end{tabular}

the serving BS of $d_{\min }=10$ [m]. The LSF coefficient is given by $\beta_{0 l i}=\omega^{-1} d_{0 l i}^{-\alpha}$ where $d_{0 l i}$ is the distance between $\mathrm{UE}_{l i}$ and $\mathrm{BS}_{0}$ (measured in meters), $\alpha$ is the pathloss exponent, and $\omega$ is the pathloss at a reference distance of 1 meter. This LSF model is used for all three numerical study cases. The wrap-around technique is used to avoid edge effects and underestimate the interference level. Statistical channel inversion power control is used for both the pilot and data symbols, such that, $q_{l i}^{\mathrm{RP}}=\rho_{l i}^{\mathrm{RP}}=\rho / \beta_{0 l i}$ where $\rho$ is a parameter used to set the SNR level. This assumption is also used for all three numerical study cases. Table 1 contains the main parameters used for the numerical study.

Case 2) Quantized SIMO system. In Figure 8, the normalized distortion power for a quantized SIMO system with $M$ antennas at the receiver is illustrated. Here, the received signal for all samples in the coherence block is given by

$$
\mathbf{Y}=\mathbb{Q}\left(\mathbf{h} \mathbf{x}^{T}+\mathbf{N}\right) \in \mathbb{C}^{M \times \tau_{c}}
$$

where $\mathbf{x} \in \mathbb{C}^{\tau_{c} \times 1}$ is the transmitted signal (including the transmission energy per symbol) that is composed of $\tau_{p}=1$ pilot symbol and the rest correspond to data symbols. The noise is given by $\mathbf{N}$ with elements distributed as $[\mathbf{N}]_{m j} \sim$ $\mathcal{C N}\left(0, \sigma^{2}\right)$. The operator $\mathbb{Q}(\cdot)$ stands for quantization with optimized levels as in [87]. The channel estimate $\hat{h}$ is computed based on LMMSE estimation following the same approach as in Section 2.4 with observation vector given 
by the quantized vector taken from the $\tau_{p}$ column of $\mathbf{Y}$ in (42). The data estimate for an arbitrary symbol in the coherence block, denoted with the index $j$, is computed as $\hat{s}_{j}=\left(\frac{\hat{\mathbf{h}}}{\|\hat{\mathbf{h}}\|}\right)^{H}[\mathbf{Y}]_{j}$ and the normalized distortion is defined as

$$
n_{d}=\frac{\mathbb{E}\left\{\left|\hat{s}_{j}-\mathbb{E}\left\{\hat{s}_{j} s_{j}^{*}\right\} s_{j}\right|^{2}\right\}}{\sigma^{2} M}
$$

where the normalization is done with respect to the number of antennas at the receiver and the noise energy per symbols.

Case 3) Quantized single-cell MU-MIMO system. Figure 9 shows the EE of a single cell Massive MIMO system with imperfect CSI where the BS deploys $M$ antennas and serves $K$ UEs. The quantization is implemented with optimized levels as in [87] and the received signal at the BS is

$$
\mathbf{Y}=\mathbb{Q}\left(\sum_{i=1}^{K} \mathbf{h}_{i} \mathbf{x}_{i}^{T}+\mathbf{N}\right) \in \mathbb{C}^{M \times \tau_{c}}
$$

where $\mathbf{x}_{i}$ encompass the transmitted symbols (i.e., pilots and data) from $\mathrm{UE}_{i}$ (including the transmission energy per symbol), and $\mathbf{h}_{i} \sim \mathcal{C N}\left(\mathbf{0}, \mathbf{I}_{M} \beta_{i}\right)$ is the channel between $\mathrm{UE}_{i}$ and the BS. LMMSE channel estimation is used following the same approach as in Section 2.4 and the SE is computed based on the capacity lower bound in (29) and (30). The power consumption is given by (33). Table 1 depicts the values of the parameters used for the numerical results.

In what follows, the main characteristics of Massive MIMO are explained based on the results in Section 2.5, (34), (40), and Figures 7-9.

Spatial multiplexing. The $\mathrm{SE}$ in (40) corresponds to $\mathrm{UE}_{0 k}$ and $K$ parallel data streams are transmitted simultaneously in each cell. Thus, the sum SE in cell 0 is $\sum_{i=1}^{K} \mathrm{R}_{0 i}$. By assuming a power control scheme for UEs in cell 0 that provides uniform rate, the sum rate in cell 0 is then $\sum_{i=1}^{K} \mathrm{R}_{0 i}=K \mathrm{R}_{0}$ which grows linearly with $K$. In Figure 7, the effect of spatial multiplexing is exemplified by comparing the result for serving a single UE per cell with $M=50$ (gray bottom line) and the rest of the curves.

Coherent processing gain. The numerator of the SINR inside the logarithm in (40) scales linearly with the number of BS antennas and the $\mathrm{SE}$ is an increasing function of $M$. In a single cell scenario $\mathcal{P}_{0 k}^{\mathrm{RP}}=(0, k)$, which means that the SINR scales linearly with $M$ and the SE scales 


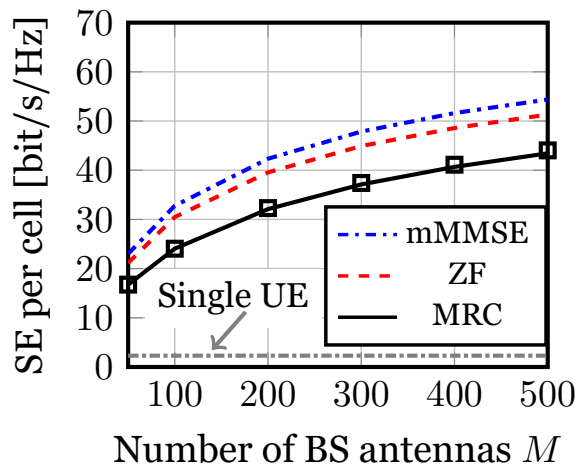

(a) Low SNR, $10 \log _{10}\left(\frac{\rho}{\sigma^{2}}\right)=-6[\mathrm{~dB}]$.

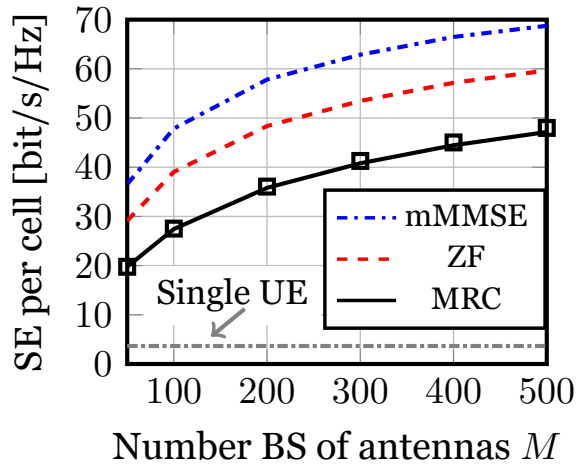

(b) High SNR, $10 \log _{10}\left(\frac{\rho}{\sigma^{2}}\right)=10[\mathrm{~dB}]$.

Figure 7: SE per cell versus $M$ in a multicell scenario for $K=10$ and $\tau_{p}=10$. The lines correspond to (31) and the squared marks follow from (40).

logarithmically with $M$. This result is exemplified in Figure 7 for a multicell scenario where it is shown that the SE in an increasing function of $M$.

Pilot contamination. This phenomenon causes three key effects: $i$ ) It decreases the estimation quality by reducing $\gamma_{0 k}$. ii) It decreases the coherent gain as consequence of having lower $\gamma_{0 k}$ (see the second term in the denominator of the SINR in (40)). iii) It causes coherent interference that reduces the SE (see the first term in the denominator of the SINR in (40)).

Transmission power reduction. Scaling down the transmission data power as $\rho_{l i}^{\mathrm{RP}}=\rho_{l i} / \sqrt{M}$, results in an SINR that is still an increasing function of $M$ (see (40)). In the case of a single cell scenario, the SE scales logarithmically with $\sqrt{M}$.

Linear processing. Thanks to asymptotic favorable propagation and channel hardening, Massive MIMO with linear processing is able to provide high SE per cell, as illustrated in Figure 7. In particular, notice that in the low SNR regime (see Figure 7a) the SE with MR combining is close to the SE with ZF and mMMSE. Whereas, in the high SNR case the benefits of $\mathrm{ZF}$ and mMMSE are more pronounced.

Low end hardware. The coherent processing gain and excess number of $\mathrm{BS}$ antennas, increase the tolerance of non-coherent distortions from 


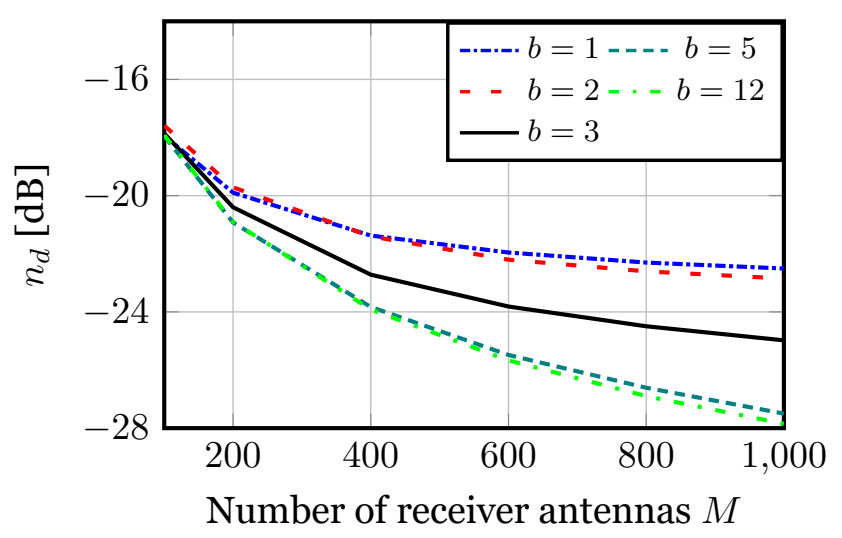

Figure 8: Normalized distortion power versus $M$ for a quantized SIMO system with $10 \log _{10}\left(\frac{\rho}{\sigma^{2}}\right)=0[\mathrm{~dB}]$.

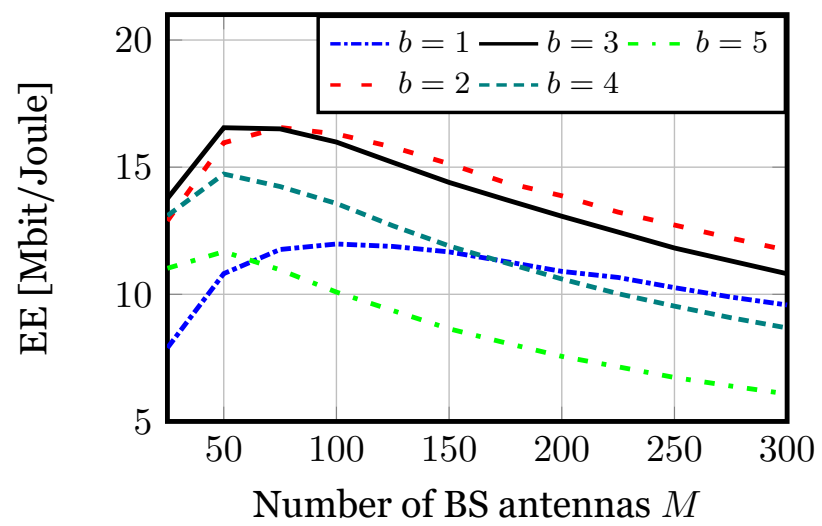

Figure 9: EE versus $M$ with $K=10$ and $10 \log _{10}\left(\frac{\rho}{\sigma^{2}}\right)=-6[\mathrm{~dB}]$ for a quantized single-cell Massive MIMO.

low end hardware. In the case of quantization, this result is exemplified in Figure 8 where the distortion power normalized by $M$ decreases as $M$ grows large. Notice that the power consumption of ADCs increases exponentially with the ADC bit resolution (see (39)), thus Massive MIMO is also able to reduce circuit power by relaxing the ADC bit resolution. As a results, high EE can by achieved by Massive MIMO which is illustrated in Figure 9. 


\section{Chapter 3}

\section{Alternative Massive MIMO Designs}

This chapter introduces the alternative designs for Massive MIMO studied in this dissertation. The system model assumptions done to analyze these designs are described and motivated while the main insights from the analysis are explained.

In Section 3.1, the SP method is investigated. First, in Section 3.1.1, the key principles of SP channel estimation are analyzed. Second, in Section 3.1.2 a comparison between RP and SP is given in terms of SE. Third, in Section 3.1.3, the impact of UL power control on the SE with SP is analyzed. Up to this point all the analysis considers only i.i.d. Rayleigh channels. Finally, in Section 3.1.4, the SE benefits of the MICED algorithm are compared between RP and SP under correlated Rayleigh fading channels.

In Section 3.2, the mixed-ADCs method is investigated. First, in Section 3.2.1, the models for hardware distortion are introduced and motivated. Second, in Section 3.2.2, the optimal tradeoff between the hardware distortion and the number of BS antennas is analyzed. Third, in Section 3.2.3, the optimal ADC bit allocation to maximize SE and minimize the power consumption is studied using majorization theory. Up to this point, the investigation considers only i.i.d. Rayleigh channels. Finally, in Section 3.2.4, the optimal ADC bit allocation to minimize the pilot distortion, maximize the SINR or minimize the power consumption is studied under correlated Rayleigh fading with LSF variations among the UL channels towards different BS antennas. 


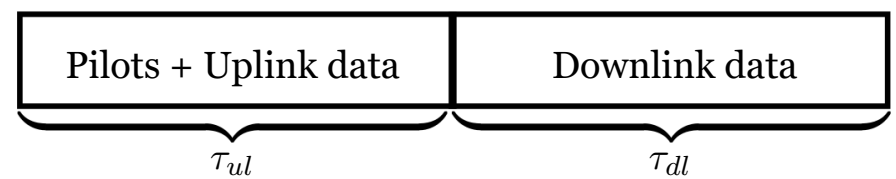

Figure 10: TDD transmission protocol with SP.

\subsection{Superimposed Pilots}

The main idea behind SP is to eliminate the pilot overhead and reduce the pilot contamination effect to offer a higher SE than with RP. To study SP, a TDD transmission protocol is assumed, as depicted in Figure 10, where the pilot and data symbols are superimposed in the UL. This means that in each UL channel use (i.e., each sample on the UL part of the coherence block) a linear combination of one pilot and one data symbols is sent by each UE. The channel estimates are constructed from the received signal in the UL and, in turn, are used for UL and DL signal processing via channel reciprocity.

Consider a multicell MU-MIMO system model, similar to the one in Section 2.4, where each BS deploys $M$ antennas and $K$ UEs are served in each cell. A set $\mathcal{V}_{\tau_{u l}}$ of mutually orthogonal pilot sequences with cardinality $\tau_{u l}$ is available for channel estimation. The pilot used by $\mathrm{UE}_{0 k}$ is $\varphi_{0 k} \in \mathcal{V}_{\tau_{u l}}$ with elements having unit modulus, that is, $\left|\left[\varphi_{0 k}\right]_{j}\right|=1 \forall j \in\left\{1, \ldots, \tau_{u l}\right\}$. Different pilots are assigned within each individual cell, which means that they are mutually orthogonal, that is

$$
\boldsymbol{\varphi}_{0 k}^{H} \boldsymbol{\varphi}_{0 i}= \begin{cases}\tau_{u l} & \text { for } k=i \\ 0 & \text { for } k \neq i\end{cases}
$$

In the case of SP, there are more pilot sequences available than with RP since SP pilots cover the whole UL coherence block (i.e., $\tau_{u l}$ pilot symbols), whereas with RP, only a fraction of it is used (i.e., $\tau_{p}<\tau_{u l}$ pilot symbols). However, the number of pilots is still limited by the size of the UL coherence block and therefore, in dense scenarios with many cells and UEs per cell, pilots would still need to be reused between cells. The set $\mathcal{P}_{0 k}^{\text {SP }}$ encloses the indices of all UEs that use the same pilot as $\mathrm{UE}_{0 k}$, including itself, and is defined as

$$
\mathcal{P}_{0 k}^{\mathrm{SP}}=\left\{(l, i): \varphi_{0 k}^{H} \boldsymbol{\varphi}_{l i} \neq 0\right\} .
$$

Here, $i \in\{1, \ldots, K\}$ and $l \in \Phi$ where $\Phi$ is the set containing the indices of all BSs. The received signal in the $\mathrm{UL}$ at $\mathrm{BS}_{0}$ is 


$$
\mathbf{Z}_{0}=\sum_{l \in \Phi} \sum_{i=1}^{K} \mathbf{h}_{0 l i}\left(\sqrt{q_{l i}^{\mathrm{SP}}} \boldsymbol{\varphi}_{l i}^{T}+\sqrt{\rho_{l i}^{\mathrm{SP}}} \mathbf{s}_{l i}^{T}\right)+\mathbf{N}_{0} \in \mathbb{C}^{M \times \tau_{u l}}
$$

where $\mathbf{s}_{l i} \in \mathbb{C}^{\tau_{u l} \times 1}$ is the vector composed of the UL data symbols transmitted by $\mathrm{UE}_{l i}$. The noise matrix is $\mathbf{N}_{0}=\left[\mathbf{n}_{01}, \ldots, \mathbf{n}_{0 \tau_{u l}}\right]$ with $\mathbf{n}_{0 j} \sim \mathcal{C N}\left(\mathbf{0}, \mathbf{I}_{M} \sigma^{2}\right)$ and $\sigma^{2}$ is the average noise energy per symbol. The transmission energy per pilot symbol from $\mathrm{UE}_{l i}$ is $q_{l i}^{\mathrm{SP}}$, and the average transmission energy per data symbol is $\rho_{l i}^{\text {SP }}$. Note that, with SP the pilot and data symbols are transmitted simultaneously. Thus, the transmission power needs to be shared between pilot and data symbols, so that $q_{l i}^{\mathrm{SP}}+\rho_{l i}^{\mathrm{SP}}$ is the total average transmission energy per symbol from $\mathrm{UE}_{l i}$.

\subsubsection{LMMSE Channel Estimation}

To estimate the channels, it is assumed that the channel, noise and data probability density functions are known, but not their random realizations. In addition, all deterministic quantities are assumed to be known, such as, the pilot sequences and transmission energy per symbol.

LMMSE channel estimation is used where the observations are given by performing a de-spreading operation on $\mathbf{Z}_{0}$ such that

$$
\begin{aligned}
\mathbf{z}_{0 k} & =\mathbf{Z}_{0} \frac{\varphi_{0 k}^{*}}{\tau_{u l} \sqrt{q_{0 k}^{\mathrm{SP}}}} \\
= & \sum_{(l, i) \in \mathcal{P}_{0 k}^{\mathrm{SP}}} \mathbf{h}_{0 l i} \sqrt{\frac{q_{l i}^{\mathrm{SP}}}{q_{0 k}^{\mathrm{SP}}}}+\underbrace{\sum_{l \in \Phi} \sum_{i=1}^{K} \mathbf{h}_{0 l i} \sqrt{\frac{\rho_{l i}^{\mathrm{SP}}}{q_{0 k}^{\mathrm{SP}}} \frac{\mathbf{s}_{l i}^{T}}{\tau_{u l}}}}_{\begin{array}{c}
\text { Interference in channel } \\
\text { estimates due to data symbols }
\end{array}}+\mathbf{N}_{0} \frac{\boldsymbol{\varphi}_{0 k}^{*}}{\tau_{u l} \sqrt{q_{0 k}^{\mathrm{SP}}}} \in \mathbb{C}^{M \times 1} .
\end{aligned}
$$

The following lemma summarizes the results for obtaining the LMMSE channel estimates of $\mathbf{h}_{00 k}$ under i.i.d. Rayleigh fading channels.

Lemma 2. The LMMSE estimate of $\mathbf{h}_{00 k}$ under i.i.d. Rayleigh fading with $S P$ based on $\mathrm{z}_{0 k}$ is

$$
\hat{\mathbf{h}}_{00 k}=\gamma_{0 k} \mathbf{z}_{0 k}
$$

where

$$
\gamma_{0 k}=\frac{q_{0 k}^{\mathrm{SP}} \tau_{u l} \beta_{00 k}}{\sum_{(l, i) \in \mathcal{P}_{0 k}^{\mathrm{Sp}}} q_{l i}^{\mathrm{SP}} \tau_{u l} \beta_{0 l i}+\sum_{l \in \Phi} \sum_{i=1}^{K} \rho_{l i}^{\mathrm{SP}} \beta_{0 l i}+\sigma^{2}} .
$$


The covariance of the channel estimate is

$$
\mathbb{E}\left\{\hat{\mathbf{h}}_{00 k} \hat{\mathbf{h}}_{00 k}^{H}\right\}=\gamma_{0 k} \beta_{00 k} \mathbf{I}_{M} .
$$

Proof. It follows from computing the linear channel estimate of $\mathbf{h}_{00 k}$ that minimizes the MSE, based on the observations $\mathbf{z}_{0 k}$ in (48). This result is based on applying standard LMMSE estimation techniques [80, Ch. 15.8] to the problem at hand.

To gain insights into the SE performance comparison between RP and $\mathrm{SP}$, understanding the differences between the channel estimates with RP and SP is essential. These differences are summarized as follows:

\section{SP has more de-spreading gain.}

Since with SP the whole UL part of the coherence block is used for both pilots and data, the number of observations available for channel estimation is higher than with RP, that is, $\tau_{u l} \geq \tau_{p}$. This means that the numerator in (50) with SP, referred to as the de-spreading gain, is higher than the numerator in (41) with RP.

\section{Channel estimates with SP have less interference from pilot con- tamination.}

The effect of pilot contamination in the channel estimates depends on the UEs belonging to the set $\mathcal{P}_{0 k}^{\mathrm{SP}}$, as shown in the first term in the denominator of (50) with SP, or to the set $\mathcal{P}_{0 k}^{\mathrm{RP}}$, in the first term in the denominator of (41) with RP. Since the number of available pilots is higher with SP than with RP, the pilots can be reused less frequently in the network. Thus, the cardinality of the set $\mathcal{P}_{0 k}^{\mathrm{SP}}$ is lower than the one of the set $\mathcal{P}_{0 k}^{\mathrm{RP}}$. In addition, if a good pilot allocation is done such that the same pilots are reused in cells further apart as the number of available pilots increases. Then, the pathloss between $\mathrm{BS}_{0}$ and the UEs in the set $\mathcal{P}_{0 k}^{\mathrm{SP}}$ is higher than for UEs in the set $\mathcal{P}_{0 k}^{\mathrm{RP}}$. As a result, the effect of the pilot contamination is reduced with SP compared to RP.

\section{Channel estimates with SP have interference from data symbols that reduces the estimation quality.}

As shown in the second term in (48), the observations with SP (after despreading) have an extra amount of interference introduced by the data symbols, which does not exist with RP. This interference reduces the quality of the channel estimates which appears as the second term in the denominator of (50). Notice also that this interference comes from all UEs in the system, including the UE of interest itself. We stress that 
the presence of this data interference in (48) changes the distribution of the observation vector $\mathbf{z}_{0 k}$ which is no longer Gaussian, but depends on the product of the channel and data symbols. This makes the analytical derivation of the MMSE estimator in closed-form less tractable and valuable insights tend to be lost in complicated expressions. For this reason in this dissertation, LMMSE channel estimation is used instead. This means that the LMMSE channel estimates with SP are not coinciding with the MMSE channel estimates as it does in the case with RP. In addition, the channel estimates with SP and their corresponding estimation errors are uncorrelated but not statistically independent. This fact calls for a different analysis from the one in $[7,8]$, when computing closed-form expressions for the effective SINR in (31).

\section{The interference from data creates correlation between channel estimates and data symbols.}

The observations $\mathbf{z}_{0 k}$ depend on the data signals through the second term in (48), and thus, the channel estimates and data symbols are correlated. This means that when the channel estimates are used for linear signal processing to obtain the data estimates, extra interference terms appear which are caused by the correlation between data symbols and channel estimates. This phenomena is similar to pilot contamination, but caused in a different way.

\subsubsection{Spectral Efficiency}

The detection is based on maximum ratio linear processing due to its low complexity, good performance in low SNR scenarios (see Section 2.6), and analytical tractability with non-Gaussian channel estimates. The SE expressions are based on the analysis given in Section 2.5. Notice that with SP, the UL signal for both the pilot estimation and data detection is the same, see (47). Since the pilot symbols are known at the BS, they can be subtracted from the received signal by using the knowledge from the channel estimates, which may be useful to reduce interference. This effect is studied in two cases: $i$ ) The channel estimates are used to subtract the estimated versions of the received pilot symbols such that the received signal for data detection processing is

$$
\mathbf{Z}_{0}=\sum_{l \in \Phi} \sum_{i=1}^{K}\left(\left(\mathbf{h}_{0 l i}-\hat{\mathbf{h}}_{0 l i}\right) \sqrt{q_{l i}^{\mathrm{sP}}} \boldsymbol{\varphi}_{l i}^{T}+\mathbf{h}_{0 l i} \sqrt{\rho_{l i}^{\mathrm{SP}}} \mathbf{s}_{l i}^{T}\right)+\mathbf{N}_{0} \in \mathbb{C}^{M \times \tau_{u l}}
$$


ii) The received pilot symbols are perfectly removed from the received signal for the data detection process, that is,

$$
\mathbf{Z}_{0}=\sum_{l \in \Phi} \sum_{i=1}^{K} \mathbf{h}_{0 l i} \sqrt{\rho_{l i}^{\text {sp }}} \mathbf{s}_{l i}^{T}+\mathbf{N}_{0} \in \mathbb{C}^{M \times \tau_{u l}} .
$$

The first case in (52) corresponds to a more practical approach to see what are the gains from subtracting the pilots. However, the analytical expressions become involved and little insights can be obtained from them, thus only numerical results are provided for this case. In the second case, the received pilot symbols are perfectly removed in order to obtain an upper bound on the performance that can be achieved with SP. ${ }^{1}$ In what follows, the signal processing with SP is referred to as: "no pilot subtraction" when (47) is taken as the received data signal, "estimated pilot subtraction" when (52) is used, and "perfect pilot subtraction" when using (53).

The assignment of pilot sequences among cells affects the pilot contamination and makes it difficult to obtain a clear comparison between the performance of RP and SP. Thus, a random pilot allocation strategy is introduced to obtain clear insights into the effect of the pilot length on the pilot contamination with both SP and RP. This is implemented by having each BS selecting $K$ mutually orthogonal pilots at random and assigning them to the UEs in its cell. This means that

$$
\begin{aligned}
& \text { with RP } \\
& \phi_{0 k}^{H} \phi_{l i}=\left\{\begin{array}{ll}
\tau_{p} & \text { for } l=0 \text { and } k=i \\
0 & \text { for } l=0 \text { and } k \neq i \\
\tau_{p} & \text { for } l \neq 0 \text { with } \\
\text { probability } \frac{1}{\tau_{p}} & \text { for } l \neq 0 \text { with } \\
\text { probability } 1-\frac{1}{\tau_{p}},
\end{array} \quad \quad \boldsymbol{\varphi}_{0 k}^{H} \varphi_{l i}= \begin{cases}\tau_{u l} & \text { for } l=0 \text { and } k=i \\
0 & \text { for } l=0 \text { and } k \neq i \\
\tau_{u l} & \text { for } l \neq 0 \text { with } \\
\text { probability } \frac{1}{\tau_{u l}} & \text { for } l \neq 0 \text { with } \\
0 & \text { probability } 1-\frac{1}{\tau_{u l}} .\end{cases} \right.
\end{aligned}
$$

The probability that in a given cell $l \neq 0$ there is a UE with the same pilot as $\mathrm{UE}_{0 k}$ is $K / \tau_{p}$ with RP, and $K / \tau_{u l}$ with SP. In the case where in said cell $l \neq 0$ there is such a UE, the individual selection of this UE is done uniformly at

\footnotetext{
${ }^{1}$ Note that this is, however, an upper bound on a lower bound on the capacity.
} 
random. Based on these assumptions, the following results hold

$\mathbb{E}\left\{\sum_{(l, i) \in \mathcal{P}_{0 k}^{\mathrm{RP}} \backslash(0, k)} q_{l i}^{\mathrm{RP}} \beta_{0 l i}\right\}=\mathbb{E}\left\{\sum_{l \in \Phi \backslash 0} \sum_{i=1}^{K} \frac{\phi_{l i}^{H} \phi_{0 k}}{\tau_{p}} q_{l i}^{\mathrm{RP}} \beta_{0 l i}\right\}=\sum_{l \in \Phi \backslash 0} \frac{K}{\tau_{p}} \frac{1}{K}\left(\sum_{i=1}^{K} q_{l i}^{\mathrm{RP}} \beta_{0 l i}\right)$,

$\mathbb{E}\left\{\sum_{(l, i) \in \mathcal{P}_{0 k}^{\mathrm{sp}} \backslash(0, k)} q_{l i}^{\mathrm{SP}} \beta_{0 l i}\right\}=\mathbb{E}\left\{\sum_{l \in \Phi \backslash 0} \sum_{i=1}^{K} \frac{\varphi_{l i}^{H} \varphi_{0 k}}{\tau_{u l}} q_{l i}^{\mathrm{SP}} \beta_{0 l i}\right\}=\sum_{l \in \Phi \backslash 0} \frac{K}{\tau_{u l}} \frac{1}{K}\left(\sum_{i=1}^{K} q_{l i}^{\mathrm{sP}} \beta_{0 l i}\right)$.

The combining vector is defined as $\mathbf{v}_{00 k}=\left(\gamma_{0 k} \sqrt{M \beta_{00 k}}\right)^{-1} \hat{\mathbf{h}}_{00 k}$, where $\gamma_{0 k}$ is given in (41) with RP and in (50) with SP. The scaling factor $\left(\gamma_{0 k} \sqrt{M \beta_{00 k}}\right)^{-1}$ is selected to provide an equivalent gain of $\left|\mathbb{E}\left\{\mathbf{v}_{00 k}^{H} \mathbf{h}_{00 k}\right\}\right|^{2}=M \beta_{00 k}$ (found in the numerator of SINR expressions in (60) and (64)) for the desired signal with both RP and SP methods. As a result, the impact of channel estimation quality in the achievable SE expressions is given by

$$
\gamma_{0 k}^{\mathrm{RP}}=\mathbb{E}\left\{\left.\frac{1}{\gamma_{0 k}}\right|_{\text {eq. (41) }}\right\}^{-1}=\frac{q_{0 k}^{\mathrm{RP}} \tau_{p} \beta_{00 k}}{q_{0 k}^{\mathrm{RP}} \tau_{p} \beta_{00 k}+\sum_{l \in \Phi \backslash 0} \sum_{i=1}^{K} q_{l i}^{\mathrm{RP}} \beta_{0 l i}+\sigma^{2}}
$$

and

$$
\gamma_{0 k}^{\mathrm{SP}}=\mathbb{E}\left\{\left.\frac{1}{\gamma_{0 k}}\right|_{\text {eq. (50) }}\right\}^{-1}=\frac{q_{0 k}^{\mathrm{SP}} \tau_{u l} \beta_{00 k}}{q_{0 k}^{\mathrm{SP}} \tau_{u l} \beta_{00 k}+\sum_{l \in \Phi \backslash 0} \sum_{i=1}^{K} q_{l i}^{\mathrm{SP}} \beta_{0 l i}+\sum_{l \in \Phi} \sum_{i=1}^{K} \rho_{l i}^{\mathrm{SP}} \beta_{0 l i}+\sigma^{2}} .
$$

These terms define indicators of the channel estimation quality under random pilot allocation with RP, and SP, where a clear dependency on $\tau_{p}$ and $\tau_{u l}$ can be identified.

In what follows, the main analytical and numerical results from Papers A and B are presented, and the main insights are explained. The analytical results are summarized in the following lemmas and theorems corresponding to the SE expressions obtained for the UL and DL with RP, and with SP, respectively.

ULSE with RP:

Lemma 3. A lower bound on the UL capacity of $\mathrm{UE}_{0 k}$ with $R P$ and $M R$ combining detection is

$$
\mathrm{R}_{0 k}^{\mathrm{UL}-\mathrm{RP}}=\left(\frac{\tau_{u l}-\tau_{p}}{\tau_{c}}\right) \log _{2}\left(1+\mathrm{SINR}_{0 k}^{\mathrm{UL}-\mathrm{RP}}\right)
$$


where $\mathrm{SINR}_{0 k}^{\mathrm{UL}-\mathrm{RP}}$ is the effective SINR of $\mathrm{UE}_{0 k}$ given by

$$
\operatorname{SINR}_{0 k}^{\mathrm{UL}-\mathrm{RP}}=\frac{M \rho_{0 k}^{\mathrm{RP}} \beta_{00 k}}{\underbrace{\frac{M}{\tau_{p}} \sum_{l \in \Phi \backslash 0} \sum_{i=1}^{K} \frac{\rho_{l i}^{\mathrm{RP}} q_{l i}^{\mathrm{RP}}}{q_{0 k}^{\mathrm{RP}}} \frac{\beta_{0 l i}^{2}}{\beta_{00 k}}}_{\begin{array}{c}
\text { Coherent interference caused } \\
\text { by pilot contamination }
\end{array}}+\underbrace{\frac{1}{\gamma_{0 k}^{\mathrm{RP}}}\left(\sum_{l \in \Phi} \sum_{i=1}^{K} \rho_{l i}^{\mathrm{RP}} \beta_{0 l i}+\sigma^{2}\right)}_{\begin{array}{c}
\text { Non-coherent interference } \\
\text { plus noise }
\end{array}}} .
$$

Proof. It follows from the analysis shown in Section 2.5 and can be found in $[7,8]$. The proof of the closed-form expression in (60) considering the effect of random pilot allocation follows from (55) and is given in Paper A.

\section{$\underline{\text { UL SE with SP: }}$}

Theorem 1. A lower bound on the UL capacity of $\mathrm{UE}_{0 k}$ with SP, MR combining, and no pilot subtraction is

$$
\mathrm{R}_{0 k}^{\mathrm{UL}-\mathrm{SP}}=\frac{\tau_{u l}}{\tau_{c}} \log _{2}(1+\underbrace{\frac{M \rho_{0 k}^{\mathrm{SP}} \beta_{00 k}}{I_{\mathrm{C}-\mathrm{I}}^{\mathrm{UL}}+I_{\mathrm{NC}-\mathrm{SP}}^{\mathrm{UL}}}}_{\mathrm{SINR}_{0 k}^{\mathrm{UL}-\mathrm{SP}}})
$$

where $\mathrm{SINR}_{0 k}^{\mathrm{UL}-\mathrm{SP}}$ is the effective SINR of $\mathrm{UE}_{0 k}$. The term $I_{\mathrm{C}-\mathrm{I}}^{\mathrm{UL}-\mathrm{SP}}$ corresponds to the coherent interference and $I_{\mathrm{NC}-\mathrm{I}}^{\mathrm{UL}-\mathrm{SP}}$ is the non-coherent interference plus noise, which are given by

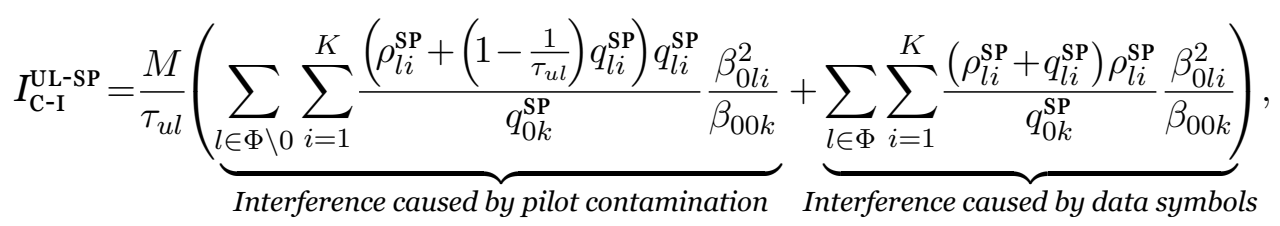

$$
\begin{aligned}
I_{\mathrm{NC}-\mathrm{I}}^{\mathrm{UL}-\mathrm{SP}}= & \frac{2}{\tau_{u l}} \rho_{0 k}^{\mathrm{SP}} \beta_{00 k}+\frac{2}{\tau_{u l}^{2}} \sum_{l \in \Phi \backslash 0} \sum_{i=1}^{K} \frac{q_{l i}^{\mathrm{SP}} \rho_{l i}^{\mathrm{SP}}}{q_{0 k}^{\mathrm{SP}}} \frac{\beta_{0 l i}^{2}}{\beta_{00 k}}+\frac{1}{\tau_{u l}^{2}} \sum_{l \in \Phi} \sum_{i=1}^{K} \frac{\left(\rho_{l i}^{\mathrm{SP}}\right)^{2}}{q_{0 k}^{\mathrm{SP}}} \frac{\beta_{0 l i}^{2}}{\beta_{00 k}} \\
& +\frac{1}{\gamma_{0 k}^{\mathrm{SP}}}\left(\sum_{l \in \Phi} \sum_{i=1}^{K}\left(q_{l i}^{\mathrm{SP}}+\rho_{l i}^{\mathrm{SP}}\right) \beta_{0 l i}+\sigma^{2}\right) .
\end{aligned}
$$

When perfect pilot subtraction is assumed, the capacity lower bound is 
given by (61) with the effective SINR defined as

$$
\begin{aligned}
\mathrm{SINR}_{0 k}^{\mathrm{UL}-\mathrm{SP}-\mathrm{UB}} & =M \rho_{0 k}^{\mathrm{SP}} \beta_{00 k} / \underbrace{\left(\frac{M}{\tau_{u l}} \sum_{l \in \Phi \backslash 0} \sum_{i=1}^{K} \frac{\rho_{l i}^{\mathrm{SP}} q_{l i}^{\mathrm{SP}}}{q_{0 k}^{\mathrm{SP}}} \frac{\beta_{0 l i}^{2}}{\beta_{00 k}}\right.}_{\begin{array}{c}
\text { Coherent interference caused } \\
\text { by pilot contamination }
\end{array}}+\underbrace{\frac{M}{\tau_{u l}} \sum_{l \in \Phi} \sum_{i=1}^{K} \frac{\left(\rho_{l i}^{\mathrm{SP}}\right)^{2}}{q_{0 k}^{\mathrm{SP}}} \frac{\beta_{0 l i}^{2}}{\beta_{00 k}}}_{\begin{array}{c}
\text { Coherent interference } \\
\text { caused by data symbols }
\end{array}} \\
& +\underbrace{\frac{1}{\tau_{u l}^{2}} \sum_{l \in \Phi} \sum_{i=1}^{K} \frac{\left(\rho_{l i}^{\mathrm{SP}}\right)^{2}}{q_{0 k}^{\mathrm{SP}}} \frac{\beta_{0 l i}^{2}}{\beta_{00 k}}+\frac{1}{\gamma_{0 k}^{\mathrm{SP}}}\left(\sum_{l \in \Phi} \sum_{i=1}^{K} \rho_{l i}^{\mathrm{SP}} \beta_{0 l i}+\sigma^{2}\right)}_{\text {Non-coherent interference plus noise }})
\end{aligned}
$$

Proof. It follows from the analysis shown in Section 2.5, combined with (56), and it is given in Paper A.

In the DL, the capacity analysis is essentially the same as the one shown in Section 2.5 with the difference that the received signal is taken at $\mathrm{UE}_{0 k}$. In this case $\mathrm{BS}_{0}$ performs a precoding operation where the transmitted signal at an arbitrary sample of the coherence block is $\mathbf{A}_{0} \mathbf{x}_{0}$. Here, $\mathbf{x}_{0}=\left[x_{01}, \ldots, x_{0 K}\right]^{T}$ is a vector containing the data symbol (including the power scaling) intended to each UE in cell 0 . The matrix $\mathbf{A}_{0} \in \mathbb{C}^{M \times K}$ is called the precoding matrix and it is meant to process the data symbols in such a way that the signals containing $x_{0 k}$ add coherently at the location of $\mathrm{UE}_{0 k}$. This allows $K$ parallel data streams to be supported in each coherence block. In the following analysis, MR transmission processing is assumed, where the precoding matrix is selected as $\mathbf{A}_{0}=\hat{\mathbf{H}}_{0}^{*}$. The matrix $\hat{\mathbf{H}}_{0}=\left[\hat{\mathbf{h}}_{001}, \ldots, \hat{\mathbf{h}}_{00 K}\right]$ is comprised of the UL channel estimates of all UEs served by $\mathrm{BS}_{0}$ which can be used for DL precoding by virtue of channel reciprocity. The channel estimates with SP are given in (49) and with RP in (22). ${ }^{2}$ The SE expressions in the DL with $\mathrm{RP}$, and $\mathrm{SP}$ are given as follows.

DL SE with RP:

Lemma 4. A lower bound on the DL capacity of $\mathrm{UE}_{0 k}$ with $R P$ and $M R$ precoding is

$$
\mathrm{R}_{0 k}^{\mathrm{DL}-\mathrm{RP}}=\frac{\tau_{d l}}{\tau_{c}} \log _{2}\left(1+\mathrm{SINR}_{0 k}^{\mathrm{DL}-\mathrm{RP}}\right)
$$

\footnotetext{
${ }^{2}$ Note that (22) defines the MMSE channel estimate of $\hat{\mathbf{h}}_{00 k}$ with RP for correlated Rayleigh channels. In the case of i.i.d. Rayleigh fading, which is assumed in this analysis, $\mathbf{R}_{00 k} \boldsymbol{\Psi}_{00 k}{ }^{-1}=\gamma_{0 k} \mathbf{I}_{M}$.
} 
where $\mathrm{SINR}_{0 k}^{\mathrm{DL}-\mathrm{RP}}$ is the effective SINR of $\mathrm{UE}_{0 k}$ given by

$$
\mathrm{SINR}_{0 k}^{\mathrm{DL}-\mathrm{RP}}=\frac{M p_{0 k}^{\mathrm{RP}} \beta_{00 k} \gamma_{0 k}^{\mathrm{RP}}}{\frac{M}{\tau_{p}^{\mathrm{RP}}} \sum_{l \in \Phi \backslash 0} \sum_{i=1}^{K} \frac{q_{0 k}^{\mathrm{RP}} p_{l i}^{\mathrm{RP}}}{q_{l i}^{\mathrm{RP}}} \frac{\beta_{l 0 k}^{2} \gamma_{l i}^{\mathrm{RP}}}{\beta_{l l i}}+\sum_{l \in \Phi} \beta_{l 0 k} \sum_{i=1}^{K} p_{l i}^{\mathrm{RP}}+\sigma^{2}} .
$$

The average transmission energy per symbol allocated by $\mathrm{BS}_{l}$ to $\mathrm{UE}_{l i}$ with $R P$ is $p_{l i}^{\mathrm{RP}}$.

\section{DLSE with $S P$ :}

Theorem 2. A lower bound on the DL capacity of $\mathrm{UE}_{0 k}$ with $S P$ and $M R$ precoding is

$$
\mathrm{R}_{0 k}^{\mathrm{DL}-\mathrm{SP}}=\frac{\tau_{d l}}{\tau_{c}} \log _{2}(1+\underbrace{\frac{M p_{0 k}^{\mathrm{SP}} \beta_{00 k} \gamma_{0 k}^{\mathrm{SP}}}{I_{\mathrm{C}-\mathrm{I}}^{\mathrm{DL}}+I_{\mathrm{NC}-\mathrm{I}}^{\mathrm{DL}}}}_{\mathrm{SINR}_{0 k}^{\mathrm{DL}-\mathrm{SP}}})
$$

where $\mathrm{SINR}_{0 k}^{\mathrm{DL}-\mathrm{SP}}$ is the effective SINR of $\mathrm{UE}_{0 k}$. The term $I_{\mathrm{C}-\mathrm{I}}^{\mathrm{DL}}$ corresponds to the coherent interference and $I_{\mathrm{NC}-\mathrm{I}}^{\mathrm{DL}}$ is the non-coherence interference plus noise, which are given by

$$
I_{\mathrm{C}-\mathrm{I}}^{\mathrm{DL}-\mathrm{SP}}=\underbrace{\frac{M}{\tau_{u l}} \sum_{l \in \Phi \backslash 0} \sum_{i=1}^{K} \frac{q_{0 k}^{\mathrm{SP}} p_{l i}^{\mathrm{SP}}}{q_{l i}^{\mathrm{SP}}} \frac{\beta_{l 0 k}^{2} \gamma_{l i}^{\mathrm{SP}}}{\beta_{l l i}}}_{\text {Interference caused by pilot contamination }}+\underbrace{\frac{M}{\tau_{u l}} \sum_{l \in \Phi} \sum_{i=1}^{K} \frac{\rho_{0 k}^{\mathrm{SP}} p_{l i}^{\mathrm{SP}}}{q_{l i}^{\mathrm{SP}}} \frac{\beta_{l 0 k}^{2} \gamma_{l i}^{\mathrm{SP}}}{\beta_{l l i}}}_{\text {Interference caused by data symbols }}
$$

$$
I_{\mathrm{NC}-\mathrm{I}}^{\mathrm{DL}-\mathrm{SP}}=\sum_{l \in \Phi} \beta_{l 0 k} \sum_{i=1}^{K} p_{l i}^{\mathrm{SP}}+\sigma^{2} .
$$

The average transmission energy per symbol allocated by $\mathrm{BS}_{l}$ to $\mathrm{UE}_{l i}$ with $S P$ is $p_{l i}^{\mathrm{SP}}$.

Proof. It can be found in Paper B.

For the numerical results, a multicell MU-MIMO random network deployment is considered. The BSs are dropped in a square area following a homogeneous Poisson point process (PPP) with a density of $100\left[\mathrm{BSs} / \mathrm{km}^{2}\right.$ ] where the area is properly scaled to simulate an average number of $30 \mathrm{BSs}$. The wraparound technique is used to avoid cell edge effects. $K$ UEs per cell are distributed uniformly within the Voronoi region around each BS. The LSF is modeled as $\beta_{l l k}=\omega^{-1} d_{l l k}^{-\alpha}$ where $d_{l l k}[\mathrm{~m}]$ is the distance between $\mathrm{BS}_{l}$ and $\mathrm{UE}_{l k}$ (with minimum distance $d_{\min }=10[\mathrm{~m}]$ ), $\alpha$ is the pathloss exponent and 
$\omega$ is the pathloss at a reference distance of $1[\mathrm{~m}]$. The selection of simulation parameters is depicted in Table 1 (found in Section 2.6).

In cases where several UEs are served in the UL, the transmission power of UEs needs to be controlled in order to avoid that signals from UEs close to the BSs overwhelm signals from UEs further away. This is particularly important in Massive MIMO where low-resolution ADCs are expected to be used $[36,88]$. Thus, statistical channel inversion power control is assumed where the transmission energy per symbol is selected to invert the LSF gain of the channel and provide the same average received energy per symbol on each antenna for all UEs. That is,

$$
q_{l i}^{\mathrm{RP}}=\rho_{l i}^{\mathrm{RP}}=\frac{\varrho_{u l}}{\beta_{l l i}}, \quad q_{l i}^{\mathrm{SP}}=\frac{\Delta \varrho_{u l}}{\beta_{l l i}}, \quad \rho_{l i}^{\mathrm{SP}}=\frac{(1-\Delta) \varrho_{u l}}{\beta_{l l i}},
$$

where $\Delta \in[0,1]$ is the proportion between the power allocated to pilot and data symbols with SP. Thus, the average received energy per symbol at an arbitrary antenna element $m$ of $\mathrm{BS}_{l}$, for $\mathrm{UE}_{l i}$ is

$$
\begin{aligned}
& \mathbb{E}\left\{\left|\left[\mathbf{h}_{l l i}\right]_{m}\right|^{2} q_{l i}^{\mathrm{RP}}\right\}=\mathbb{E}\left\{\left|\left[\mathbf{h}_{l l i}\right]_{m}\right|^{2} \rho_{l i}^{\mathrm{RP}}\right\}=\varrho_{u l} \\
& \mathbb{E}\left\{\left|\left[\mathbf{h}_{l l i}\right]_{m}\right|^{2} q_{l i}^{\mathrm{sP}}\right\}=\Delta \varrho_{u l}, \quad \mathbb{E}\left\{\left|\left[\mathbf{h}_{l l i}\right]_{m}\right|^{2} \rho_{l i}^{\mathrm{sP}}\right\}=(1-\Delta) \varrho_{u l} .
\end{aligned}
$$

In all results with SP, except the ones from multiobjective optimization, $\Delta$ is optimized to maximize the UL SE. Notice that the power control definition in (70) assumes that the total average energy per symbol with both RP and SP is the same, that is, $q_{l i}^{\mathrm{RP}}=\rho_{l i}^{\mathrm{RP}}=q_{l i}^{\mathrm{SP}}+\rho_{l i}^{\mathrm{SP}}$.

Before presenting the numerical results, let us introduce the concept of multiobjective optimization which is used to compare the UL and DL SE between SP and RP. A multiobjective optimization problem is characterized by having more that one mutually conflicting objectives that do not have any particular order or hierarchy [89]. In what follows a brief description of the main properties of multiobjective optimization problems is given along with their application to the UL and DL SE maximization with RP and SP.

\section{No global optimum.}

In a multiobjective optimization problem, the objectives are assumed to be mutually conflicting, this means that improving one objective will eventually lead to the detrimental of another. In addition, since there is no hierarchy among objectives, selecting the best compromise between the objectives can only be done subjectively, that is, there is no global optimum. In the context of the UL and DL SE, notice that the DL SE depends on the channel estimation quality. In the case of 
$\mathrm{RP}$, the DL SE is maximize by increasing the pilot length as much as possible. However, when $\tau_{p}=\tau_{u l}$ the UL SE is zero since there are no samples left in the coherence block for UL data. In the case of SP, the DL SE is maximized by allocating all the UL power towards pilot symbols, that is $\Delta=1$. However, in this case the UL SE is zero because no data symbols are transmitted.

\section{Attainable objective set.}

The set that contains all the objective values that can be achieved simultaneously, is called the attainable objective set. In the case of UL and DL SE, the attainable objective set is given by the SE expressions in Lemmas 3 and 4 with RP, and Theorems 1 and 2 with SP, such that the optimization variables satisfy: $i$ ) with $\mathrm{RP} \tau_{p} \in\left[K, \tau_{u l}\right]$ and $\tau_{u l} \in\left[K, \tau_{c}\right]$; ii) with SP $\Delta \in[0,1]$ and $\tau_{u l} \in\left[K, \tau_{c}\right]$. Notice that $\tau_{c}$ is fixed so that optimizing $\tau_{u l}$ means to optimize the proportion between UL and DL transmission in the coherence block.

\section{Pareto boundary.}

The objective values corresponding to points in the attainable objective set for which no objective can be further improved without deteriorating another one, is called the Pareto boundary. This is the closest notion to optimality in multiobjective optimization. Thus, the ideal operating point should lie somewhere along the Pareto boundary, and which point is selected would depend on the design requirements of the network. In the case of UL and DL SE optimization, the Pareto boundary indicates the optimal compromise between UL and DL SE.

In what follows the numerical results comparing RP and SP in terms of SE are presented. To ease readability, a summary of the abbreviations used for the legends in Figures 11-13 is presented below:

- RP with pilot length equal to the number of UEs per cell, i.e., $\tau_{p}=K$ is denoted as "RP $\tau_{p}=K$ ";

- RP with optimal pilot length to maximize the SE is denoted as " $\mathrm{RP} \tau_{p}$ op";

- SP with no pilot subtraction is denoted as "SP no-sub";

- SP with estimated pilot subtraction is denoted as "SP e-sub";

- SP with perfect pilot subtraction is denoted as "SP p-sub". 


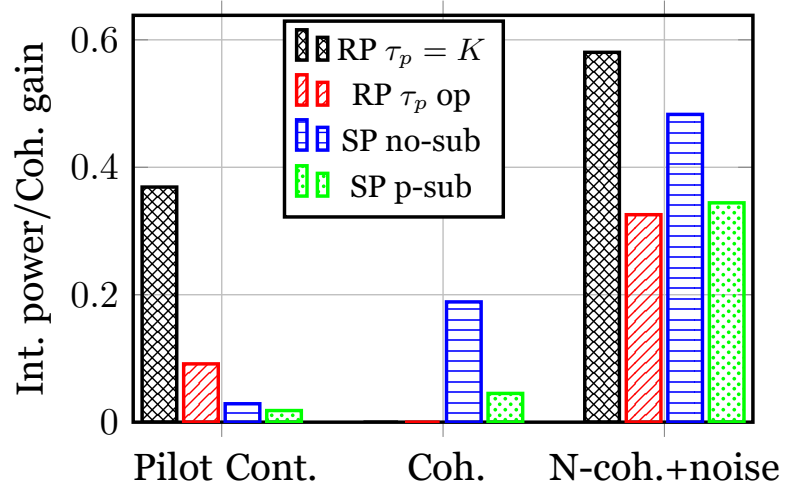

Figure 11: Interference sources in the UL for $M=100, K=10$, $10 \log _{10}\left(\frac{\underline{\varrho}_{u l}}{\sigma^{2}}\right)=0[\mathrm{~dB}]$, and $\tau_{c}=\tau_{u l}=200$.

As it was done in Section 2.6, first all the figures are introduced, and then the main insights are explained summarizing the analytical and numerical results. For the UL, Figure 11 depicts the received power from the main sources of interference normalized by the coherent gain, and Figure 12 shows the SE and EE versus the number of BS antennas. Figure 13a depicts the DL SE per cell versus the number of BS antennas when the UL is optimized to maximize the UL SE, ${ }^{3}$ for two proportions of UL and DL symbols (i.e., values of $\tau_{u l}$ and $\tau_{d l}$ when $\tau_{c}$ is fixed). In Figure 13b the Pareto boundary of UL and DL sum SE over the whole network is presented for two coherence block sizes $\left(\tau_{c}=\{25,100\}\right)$. This last results is obtained based on a multiobjective optimization analysis [89] with two objectives, that is, UL and DL network SE (i.e., the sum SE over all UEs in all BSs). The optimization variables are $\tau_{u l}, \tau_{d l}$, and $\tau_{p}$ with RP, whereas, $\Delta$ with SP. The DL power allocation is done to maximize the DL sum SE per cell and an iterative algorithm is performed over all cells until convergence is achieved. In what follows, the main insights from the analytical and numerical results are explained.

\section{SP reduces pilot contamination at the expense of adding extra co- herent interference.}

Since the pilots are reused less often in space, there are less UEs that share the same pilots and they are on average located further apart which reduces the coherent interference caused by pilot contamination. This can be seen explicitly when comparing the first term in the denominator of (60) with RP and the first term in (62) with SP, where the pilot

\footnotetext{
${ }^{3}$ The optimization is done with respect to $\tau_{p}$ with $\mathrm{RP}$ and $\Delta$ with SP.
} 


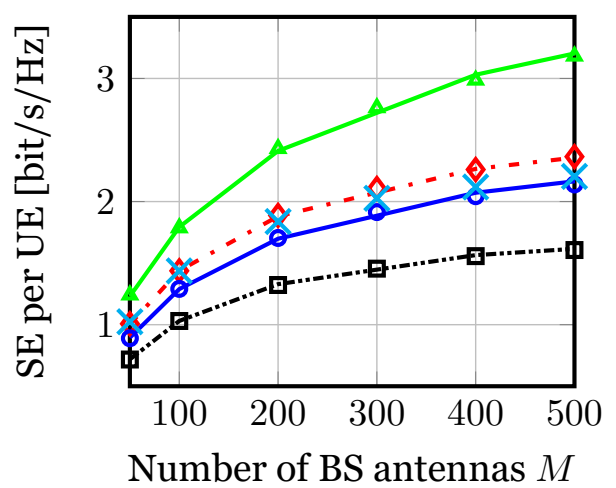

(a) SE per UE for $\tau_{c}=200, K=10$ and $10 \log _{10}\left(\frac{\underline{\varrho}_{u l}}{\sigma^{2}}\right)=-6[\mathrm{~dB}]$.

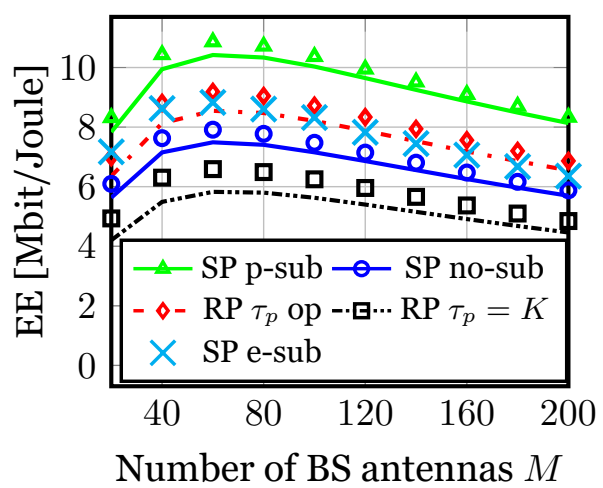

(b) $\mathrm{EE}$ for $\tau_{c}=200, \quad K=10$ and $10 \log _{10}\left(\frac{\varrho_{u l}}{\sigma^{2}}\right)=-6[\mathrm{~dB}]$.

Figure 12: SE and EE versus $M$. The markers correspond to Monte Carlo simulations over the SSF. The lines in Figure 12a correspond to the closed-form expressions in Lemma 3 and Theorem 1, whereas the lines in Figure 12b correspond to the closedform lower bounds on the SE with respect to the UE locations (see Theorem 2 in Paper A).

contamination effect is reduced by a factor of $\tau_{u l} / \tau_{p}$. In turn, coherent and non-coherent interference from data symbols is added with SP as shown in the terms (62), (63), and the denominator of (64). These effects are illustrated in Figure 11 where it can be seen that the reduction in pilot contamination with SP is followed by extra interference.

\section{Correlation between channel estimates and data limits the gains with SP resulting in comparable performance with $R P$.}

Due to the correlation between channel estimates and UL data symbols, additional coherent and non-coherent interference is introduced with SP. In turn, this effect reduces the SE and EE gains that SP offers which can be seen in Figures 12-13. The performance when both systems, RP and SP, are optimized is comparable, one cannot say that one is clearly better than another. This is shown in the UL for the SE in Figure 12a, and the EE in Figure 12b. In the case when both UL and DL and jointly optimized the DL SE with both SP and RP are also comparable as shown in Figure 13a. Furthermore, in cases when the coherence block size is significantly larger (in the order of magnitude sense) than the number of multiplexed UEs, Figure 13b shows that the Pareto boundary with SP is contained within the boundary with RP. The opposite occurs in 


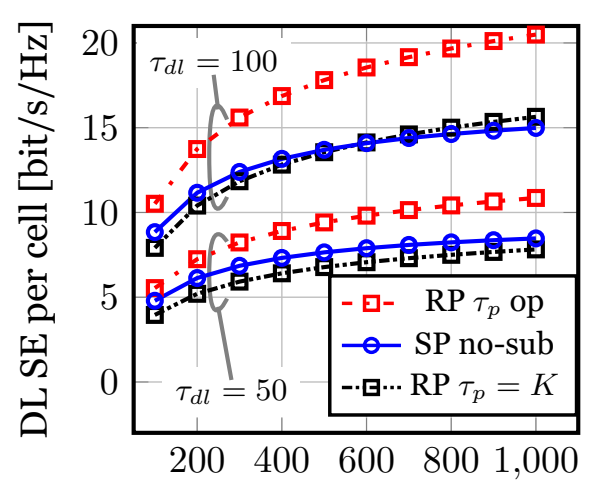

Number of BS antennas $M$

(a) $\mathrm{DL} \quad \mathrm{SE}$ per cell versus $M$ for $K=10, \quad \tau_{c}=200$ and $10 \log _{10}\left(\frac{\varrho_{u l}}{\sigma^{2}}\right)=-6[\mathrm{~dB}]$.

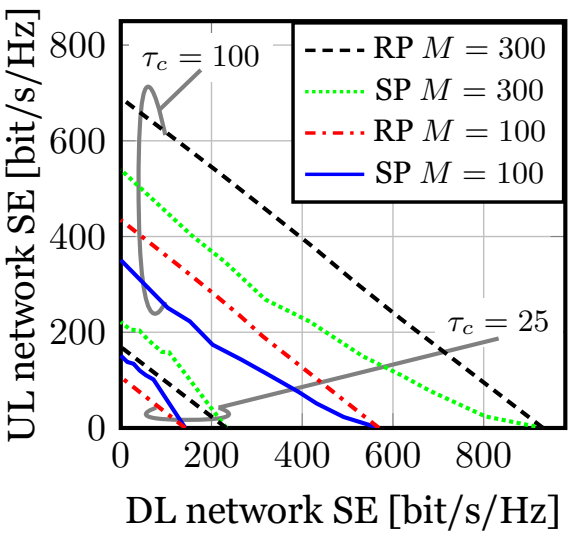

(b) Pareto boundary of UL and DL total sum network SE for $K=20$ and $10 \log _{10}\left(\frac{\varrho_{u l}}{\sigma^{2}}\right)=-6[\mathrm{~dB}]$.

Figure 13: DL SE per cell versus $M$, and Pareto boundary of UL and DL network SE. The DL power allocation is max-sum rate per cell. The maximum DL power is set so that the lowest cell edge SNR is equal to $\frac{\underline{Q}_{u l}}{\sigma^{2}}$.

extreme cases where $\tau_{c} \approx K$.

Thus, there is little incentive in using the alternative SP design to improve $\mathrm{SE}$ or EE unless the size of the coherence block is very limited and many UEs need to be multiplexed. However, in this case it may be more suitable to multiplex UEs in time or frequency, this needs further investigation and it is left for future work. On the other hand, since there is an important performance gap between SP with estimated and perfect pilot subtraction, more involved signal processing like iterative decoding algorithms could provide further gains with SP. This is investigated in Paper D and the findings are presented in Section 3.1.4.

\subsubsection{Impact of Power Control in the UL}

In the previous section, the SE with SP and statistical channel inversion power control was analyzed. However, the SE expressions given in Theorem 1 are valid for any type of power control strategy. Thus, this section studies the impact of optimal power control in the UL of a Massive MIMO system with SP, MR processing, and i.i.d. Rayleigh fading. To have a more general result, 
in contrast to Section 3.1.2, the pilot assignment among cells in this analysis is considered fixed and arbitrary.

Power control strategies are selected to manage the interference among UEs and the goal of optimizing the power depends on the design requirements of the network. As an example, the goal could be to maximize the sum SE performance, or perhaps maximizing fairness by giving all UEs the same SE. In addition, it is also important to choose optimization problem formulations that result in efficient implementations. For example, maximizing the sum SE often results in problems with high complexity that cannot be solved in polynomial time [90]. Thus, in this section the SINRs expressions are used to formulate optimization problems that can be solved efficiently in polynomial time. Consider the following optimization problem:

$$
\begin{array}{ll}
\underset{\left\{\rho_{l k}^{\mathrm{SP}}, q_{l k}^{\mathrm{S}}\right\}}{\operatorname{maximize}} & f_{\mathrm{PW}}\left(\mathrm{SINR}_{l k}\right) \\
\text { subject to } & \left(\rho_{l k}^{\mathrm{SP}}+q_{l k}^{\mathrm{SP}}\right) \leq P_{\max } \text { for } \begin{array}{l}
l \in \Phi, \\
k \in\{1, \ldots, K\},
\end{array}
\end{array}
$$

where $\mathrm{SINR}_{l k}$ is the $\mathrm{SINR}$ of $\mathrm{UE}_{l k}$ and $P_{\max }$ is the maximum transmission energy per symbol allowed per UE, assumed to be the same for all UEs. The function $f_{\mathrm{PW}}\left(\mathrm{SINR}_{l k}\right)$ corresponds to one of the following utilities:

(A) Max-min Fairness: $f_{\mathrm{PW}}\left(\mathrm{SINR}_{l k}\right)=\min _{l, k} \operatorname{SINR}_{l k}$

(B) Max-product of SINRs: $f_{\mathrm{PW}}\left(\mathrm{SINR}_{l k}\right)=\prod_{l \in \Phi k=1} \prod_{k}^{K} \operatorname{SINR}_{l k}$.

The max-min fairness approach (A) aims at providing maximum fairness in which all UEs have equal SE, whereas the max-product of SINRs (B) seeks to maximize the aggregated performance while providing some level of fairness since UEs with very small SINRs would decrease the objective. Since the SE increases logarithmically with the SINR (see (61)), the max-min fairness approach maximizes the minimum SE in the network. Moreover, the maxproduct of SINRs, also known as proportional fairness [90], maximizes a lower bound on the sum SE [8, Ch. 7].

The optimization problem in (72) with objectives (A) and (B) can be casted as geometric programming optimization problems for which efficient solvers exist $[8,91,92]$. The definition of a geometric program is given as follows:

Definition 1. Let $\mathbf{x}=\left[x_{1}, \ldots, x_{N_{x}}\right]^{T}$ with $N_{x} \in \mathbb{N}$. Then, a geometric 
program is a class of optimization problems with the following formulations

$$
\begin{array}{cl}
\underset{x_{n}>0 \forall n \in\left\{1, \ldots, N_{x}\right\}}{\operatorname{minimize}} \begin{array}{l}
\text { subject to } \\
\text { or }
\end{array} & f_{\mathrm{GP}_{0}}(\mathbf{x}) \\
\underset{x_{n}>0 \forall n \in\left\{1, \ldots, N_{x}\right\}}{\operatorname{maximize}} & f_{\mathrm{GP}_{i}}(\mathbf{x}) \leq 1 \forall i \in\left\{1, \ldots, N_{\mathrm{IC}}\right\}, \\
\text { subject to } & g_{\mathrm{GP}_{j}}(\mathbf{x})=1 \forall j \in\left\{1, \ldots, N_{\mathrm{EC}}\right\}, \\
& g_{\mathrm{GP}_{0}}(\mathbf{x}) \\
& f_{\mathrm{GP}_{i}}(\mathbf{x}) \leq 1 \forall i \in\left\{1, \ldots, N_{\mathrm{IC}}\right\}, \\
& g_{\mathrm{GP}_{j}}(\mathbf{x})=1 \forall j \in\left\{1, \ldots, N_{\mathrm{EC}}\right\} .
\end{array}
$$

The functions $f_{\mathrm{GP}_{i}}(\mathbf{x})$ for $i \in\left\{0, \ldots, N_{\mathrm{IC}}\right\}$ are posynomials and $g_{\mathrm{GP}_{j}}(\mathbf{x})$ for $j \in\left\{0, \ldots, N_{\mathrm{EC}}\right\}$ are monomials, which are defined as:

Monomial. Is a function with positive domain defined by the product of positive variables to the power of real valued exponents, that is,

$$
f_{\mathrm{MON}}(\mathbf{x})=\mathrm{u} \prod_{n=1}^{N_{x}} x_{n}^{a_{n}} \geq 0
$$

is a monomial. Here, $\mathrm{u}>0$ and $a_{n} \in \mathbb{R} \forall n \in\left\{1, \ldots, N_{x}\right\}$.

Posynomial. Is a function with positive values and corresponds to a summation of monomials, that is,

$$
f_{\mathrm{POS}}(\mathbf{x})=\sum_{i=1}^{N_{\text {PoS }}} \mathrm{u}_{i} \prod_{n=1}^{N_{x}} x_{n}^{a_{n i}} \geq 0
$$

is a posynomial with $\mathrm{u}_{i}>0$ and $a_{n i} \in \mathbb{R}$ for $n \in\left\{1, \ldots, N_{x}\right\}$ and $i \in\left\{1, \ldots, N_{\text {Pos }}\right\}$ with $N_{\text {Pos }} \in \mathbb{N}$.

A geometric program can be re-casted as a convex optimization problem on standard form by applying the variable change $y_{n}=\log \left(x_{n}\right)$, see [91] for details. In turn, convex optimization problems correspond to a mature field of optimization theory for which efficient solvers are available commercially [93,94], and the problems are solved in polynomial time [90].

In the following lemma, the effective SINR of UEs with SP is defined in terms of monomials and posynomials. 
Lemma 5. The SINR of $\mathrm{UE}_{l k}$ in Theorem 1 for a general pilot assignment with perfect and no pilot subtraction, is given by $\operatorname{SINR}_{l k}=M \rho_{l k}^{\mathrm{SP}} q_{l k}^{\mathrm{SP}} \beta_{l l k}^{2} / D_{l k}$ where

$$
\begin{aligned}
& D_{l k}=M \sum_{(\ell, i) \in \mathcal{P}_{l k}^{\mathrm{SP}} \backslash(l, k)} q_{\ell i}^{\mathrm{SP}} \rho_{\ell i}^{\mathrm{SP}} \beta_{l \ell i}^{2}+\frac{M}{\tau_{u l}} \sum_{\ell \in \Phi} \sum_{i=1}^{K}\left(a_{\ell i}+\rho_{\ell i}^{\mathrm{SP}}\right) \rho_{\ell i}^{\mathrm{SP}} \beta_{l \ell i}^{2} \\
& +\frac{2}{\tau_{u l}} \sum_{(\ell, i) \in \mathcal{P}_{l k}^{\mathrm{SP}}} a_{\ell i} \rho_{\ell i}^{\mathrm{SP}} \beta_{l \ell i}^{2}+\frac{1}{\tau_{u l}^{2}} \sum_{\ell \in \Phi} \sum_{i=1}^{K} \rho_{\ell i}^{\mathrm{SP}^{2}} \beta_{l \ell i}^{2} \\
& +\left(\sum_{(\ell, i) \in \mathcal{P}_{l k}^{\mathrm{SP}}} q_{\ell i}^{\mathrm{SP}} \beta_{l \ell i}+\frac{1}{\tau_{u l}} \sum_{\ell \in \Phi} \sum_{i=1}^{K} \rho_{\ell i}^{\mathrm{SP}} \beta_{l \ell i}+\frac{\sigma^{2}}{\tau_{u l}}\right)\left(\sum_{\ell \in \Phi} \sum_{i=1}^{K}\left(a_{\ell i}+\rho_{\ell i}^{\mathrm{SP}}\right) \beta_{l \ell i}+\sigma^{2}\right)
\end{aligned}
$$

and

$$
a_{\ell i}= \begin{cases}q_{\ell i}^{\mathrm{SP}} & \text { with no pilot subtraction } \\ 0 & \text { with perfect pilot subtraction }\end{cases}
$$

Proof. The effective SINR expression follows from the same achievable rate analysis conducted in Paper A without calculating expectations over the pilot assignment.

From Lemma 5 it can be seen that the effective SINR is a monomial function over a posynomial function of the power coefficients $q_{l k}^{\mathrm{SP}}$ and $\rho_{l k}^{\mathrm{SP}}$. Thus, the max-min fairness optimization problem can be recasted as a geometric program by doing an epigraph formulation (see Paper C for details). On the other hand, to recast the max-product of SINRs problem as a geometric program, one auxiliary variable per UE is introduced to enforce a SINR floor for each UEs as a posynomial lower than a constant (see Paper C for details).

To provide insights into the benefits that SP with power control can bring, results from Monte Carlo simulations are presented in what follows. The system model assumes 8 square cells with the BSs located at their center, and 8 UEs per cell with positions uniformly distributed at random within each cell. The LSF follows the same model as in Section 3.1.2 such that $\beta_{l l k}=\omega^{-1} d_{l l k}^{-\alpha}$, more details on the simulation setup can be found in Paper $\mathrm{C}$. To establish a benchmark, statistical channel inversion power control is included as it is defined in (70) with the proportion between pilot and data power $\Delta \in[0,1]$ optimized to maximize the sum SE of the network.

Figure 14 shows the empirical cumulative distribution function (CDF) of the SE and fraction of pilot power per UE. The benefit of max-min fairness can be seen in the low percentile values where the lowest SINR values are 

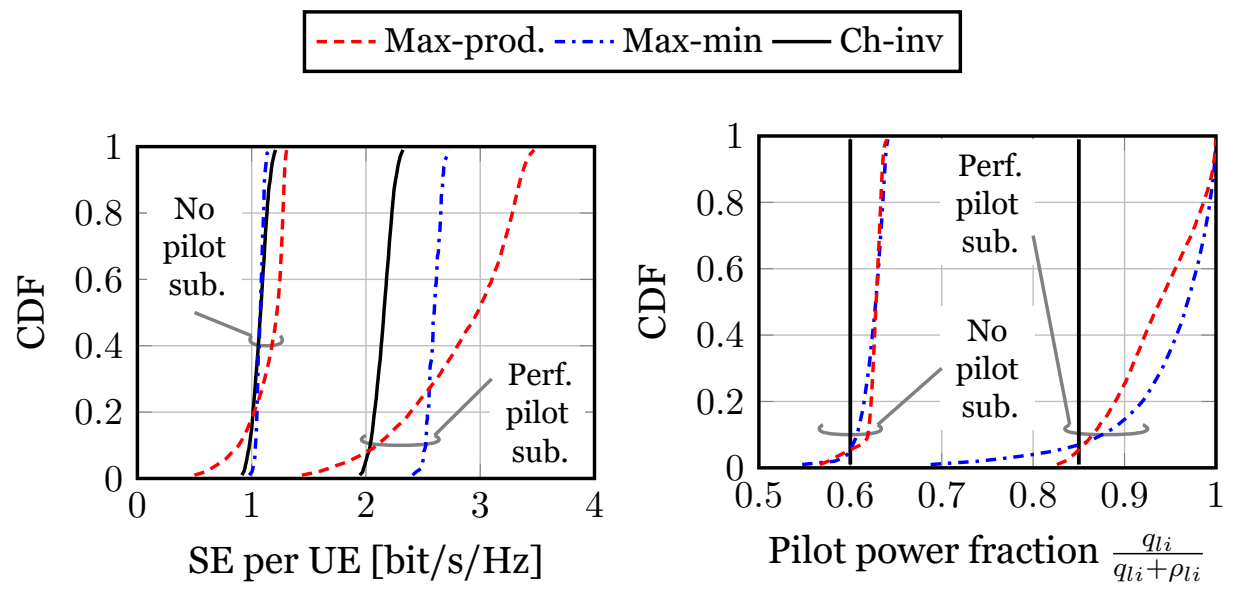

Figure 14: CDF of the SE and pilot power fraction per UE for $M=100, K=8, \tau_{u l}=32$ and $\varrho / \sigma^{2}=1(\mathrm{SNR}=0[\mathrm{~dB}])$.

higher than the other power control strategies. On the other hand, maxproduct of SINRs provides higher SE to UEs with good channel conditions (corresponding to the high percentile values) which results in higher sum SE. For the pilot power fraction, it is interesting to see that more than $60 \%$ and $80 \%$ of the power is spent on pilots for no pilot subtraction and perfect pilot subtraction, respectively. This indicates that the interference from data symbols is the main source of performance degradation and the optimal power control aims at reducing the data power to mitigate this effect.

The results also show that the impact of optimizing the power with SP is more pronounced when perfect pilot subtraction is possible. This is in line with the previous results in Section 3.1.2 suggesting that more advanced techniques to reduce the interference between pilot and data symbols can be highly beneficial in terms of SE. This is investigated in the next section.

\subsubsection{The MICED Algorithm}

In Section 3.1.2 it is shown that the SE with SP is limited by the data interference, and Section 3.1.3 shows that optimizing the transmission power does not offer higher SE unless the pilot interference is perfectly subtracted. Thus, SP has room to improve if more advanced processing is done to mitigate interference between pilots and data. In this section, the proposed MICED algorithm is introduced and analyzed to assess the potential benefits of dataaided channel estimation in the UL of a multicell Massive MIMO system 
with RP and SP methods. ${ }^{4}$ To make the analysis more general, the effect of spatially correlated fading is considered based on the system model depicted in Section 2.3.

In this dissertation, the term data-aided channel estimation refers to the use of partially decoded bits as side information to increase the channel estimation quality. Before introducing the MICED algorithm, a unified system model with RP and SP methods is defined to simplify the analysis. The terms that are left unmentioned remain with their initial definitions.

The coherence block in the UL is composed of $\tau_{c}$ complex samples, such that, $\tau_{c}=\tau_{p}+\tau_{d}$ with RP and $\tau_{c}=\tau_{p}=\tau_{d}$ with SP where $\tau_{p}$ and $\tau_{d}$ refer to the pilot and data symbols, respectively. The communication channel is modeled as correlated Rayleigh fading such that $\mathbf{h}_{l l k} \sim \mathcal{C N}\left(\mathbf{0}, \mathbf{R}_{l l k}\right)$ is the channel between $\mathrm{BS}_{l}$ and $\mathrm{UE}_{l k}$ where $\mathbf{R}_{l l k}$ is the spatial correlation matrix. The received signal at $\mathrm{BS}_{l}$ in the $\mathrm{UL}$ is

$$
\mathbf{Y}_{l}=\sum_{\ell \in \Phi} \sum_{k^{\prime}=1}^{K} \underbrace{\mathbf{h}_{l \ell k^{\prime}}}_{M \times 1} \underbrace{\mathbf{x}_{\ell k^{\prime}}^{T}}_{1 \times \tau_{c}}+\mathbf{N} \quad \in \mathbb{C}^{M \times \tau_{c}}
$$

where $\mathbf{N}=\left[\mathbf{n}_{1}, \ldots, \mathbf{n}_{\tau_{c}}\right]$ is the thermal noise with i.i.d. columns distributed as $\mathbf{n}_{j} \sim \mathcal{C N}\left(\mathbf{0}, \sigma^{2} \mathbf{I}_{M}\right) \forall j \in\left\{1, \ldots, \tau_{c}\right\}$ and $\sigma^{2}$ being the average noise energy per symbol. The signal transmitted from $\mathrm{UE}_{l k}$ is

$$
\mathbf{x}_{l k}= \begin{cases}{\left[\sqrt{q_{l k}^{\mathrm{RP}}} \boldsymbol{\phi}_{l k}^{T} \quad \sqrt{\rho_{l k}^{\mathrm{RP}}} \mathbf{s}_{l k}^{T}\right]^{T}} & \text { with RP } \\ \sqrt{q_{l k}^{\mathrm{SP}}} \boldsymbol{\varphi}_{l k}+\sqrt{\rho_{l k}^{\mathrm{SP}}} \mathbf{s}_{l k} & \text { with SP }\end{cases}
$$

where

$$
\begin{array}{ll}
q_{l k}^{\mathrm{RP}}=\rho_{l k}^{\mathrm{RP}}=\varrho_{l k} & \text { with RP, } \\
q_{l k}^{\mathrm{SP}}=\Delta_{l k} \varrho_{l k}, \rho_{l k}^{\mathrm{SP}}=\left(1-\Delta_{l k}\right) \varrho_{l k} & \text { with SP, }
\end{array}
$$

such that $\varrho_{l k}$ is the average energy per symbol with both RP and SP methods.

To estimate the channel, LMMSE channel estimation is implemented. In the case of pilot-based channel estimation (i.e., no data-aided methods applied), the following channel observations are used:

$$
\begin{aligned}
& \mathbf{z}_{l l k}^{\mathrm{RP}}=\left[\mathbf{Y}_{l}\right]_{1: \tau_{p}} \frac{\phi_{l k}^{*}}{\tau_{p} \sqrt{q_{l k}^{\mathrm{RP}}}}, \\
& \mathbf{z}_{l l k}^{\mathrm{SP}}=\mathbf{Y}_{l} \frac{\boldsymbol{\varphi}_{l k}^{*}}{\tau_{c} \sqrt{q_{l k}^{\mathrm{SP}}}} .
\end{aligned}
$$

The LMMSE channel estimates are given in the following lemma.

\footnotetext{
${ }^{4}$ Notice that RP is included as a benchmark since data-aided channel estimation is not restricted to SP.
} 
Lemma 6. Based on the observations $\mathrm{z}_{l l k}^{\mathrm{RP}}$ and $\mathrm{z}_{l l k}^{\mathrm{SP}}$, the LMMSE estimates of $\boldsymbol{h}_{l l k}$ are

$$
\begin{aligned}
& \hat{\mathbf{h}}_{l l k}^{\mathrm{RP}}=\mathbf{R}_{l l k} \Psi_{l l k}^{\mathrm{RP}-1} \mathbf{z}_{l l k}^{\mathrm{RP}} \\
& \hat{\mathbf{h}}_{l l k}^{\mathrm{SP}}=\mathbf{R}_{l l k} \Psi_{l l k}^{\mathrm{SP}}-1 \mathbf{z}_{l l k}^{\mathrm{SP}}
\end{aligned}
$$

where

$$
\begin{aligned}
\mathbf{\Psi}_{l l k}^{\mathrm{RP}} & =\underbrace{\sum_{\left(\ell, k^{\prime}\right) \in \mathcal{P}_{l k}^{\mathrm{RP}}} \mathbf{R}_{l \ell k^{\prime}} \frac{q_{\ell k^{\prime}}^{\mathrm{RP}}}{q_{l k}^{\mathrm{RP}}}}_{\text {Pilot contamination }}+\underbrace{\frac{\sigma^{2}}{q_{l k}^{\mathrm{RP}} \tau_{p}} \mathbf{I}_{M}}_{\text {Noise }}, \\
\mathbf{\Psi}_{l l k}^{\mathrm{SP}} & =\underbrace{\sum_{\left(\ell, k^{\prime}\right) \in \mathcal{P}_{l k}^{\mathrm{SP}}} \mathbf{R}_{l \ell k^{\prime}} \frac{q_{\ell k^{\prime}}^{\mathrm{SP}}}{q_{l k}^{\mathrm{SP}}}}_{\text {Pilot contamination }}+\frac{1}{\tau_{c}}(\underbrace{\sum_{\ell \in \Phi} \sum_{k^{\prime}=1}^{K} \mathbf{R}_{l \ell k^{\prime}} \frac{\rho_{\ell k^{\prime}}^{\mathrm{SP}}}{q_{l k}^{\mathrm{SP}}}}_{\text {Data interference }}+\underbrace{\left.\frac{\sigma^{2}}{q_{l k}^{\mathrm{SP}}} \mathbf{I}_{M}\right)}_{\text {Noise }} .
\end{aligned}
$$

The channel estimates are uncorrelated to the channel estimation errors which are defined as

$$
\begin{aligned}
\tilde{\mathbf{h}}_{l l k}^{\mathrm{RP}} & =\mathbf{h}_{l l k}-\hat{\mathbf{h}}_{l l k}^{\mathrm{RP}}, \\
\tilde{\mathbf{h}}_{l l k}^{\mathrm{SP}} & =\mathbf{h}_{l l k}-\hat{\mathbf{h}}_{l l k}^{\mathrm{sP}},
\end{aligned}
$$

with error correlation matrices given by

$$
\begin{aligned}
& \mathbf{C}_{l l k}^{\mathrm{RP}}=\mathbf{R}_{l l k}-\mathbf{R}_{l l k} \mathbf{\Psi}_{l l k}^{\mathrm{RP}-1} \mathbf{R}_{l l k}, \\
& \mathbf{C}_{l l k}^{\mathrm{SP}}=\mathbf{R}_{l l k}-\mathbf{R}_{l l k} \mathbf{\Psi}_{l l k}^{\mathrm{SP}}{ }^{-1} \mathbf{R}_{l l k} .
\end{aligned}
$$

Proof. It follows from employing standard LMMSE estimation techniques to the problem at hand, see Paper D for details.

To detect the data symbols the following data observations are obtained

$$
\begin{aligned}
& \underbrace{\hat{\mathbf{y}}_{l k}^{\mathrm{RP}^{T}}}_{1 \times \tau_{d}}=\underbrace{\mathbf{v}_{l k}^{H}}_{1 \times M} \underbrace{\left[\mathbf{Y}_{l}\right]_{\tau_{p}+1: \tau_{c}}}_{M \times \tau_{d}}, \\
& \underbrace{\hat{\mathbf{y}}_{l k}^{\mathrm{SP}^{T}}}_{1 \times \tau_{c}}=\underbrace{\mathbf{v}_{l k}^{H}}_{1 \times M} \underbrace{\mathbf{Y}_{l}}_{M \times \tau_{c}},
\end{aligned}
$$

where the combining vector $\mathbf{v}_{l k}$ is defined as

$$
\mathbf{v}_{l k}= \begin{cases}\hat{\mathbf{h}}_{l l k} & \text { MR } \\ \left(\sum_{k^{\prime}=1}^{K} \varrho_{l k^{\prime}}\left(\hat{\mathbf{h}}_{l l k^{\prime}} \hat{\mathbf{h}}_{l l k^{\prime}}^{H}+\mathbf{C}_{l l k^{\prime}}\right)+\sigma^{2} \mathbf{I}_{M}\right)^{-1} \hat{\mathbf{h}}_{l l k} \varrho_{l k} & \text { S-MMSE. }\end{cases}
$$




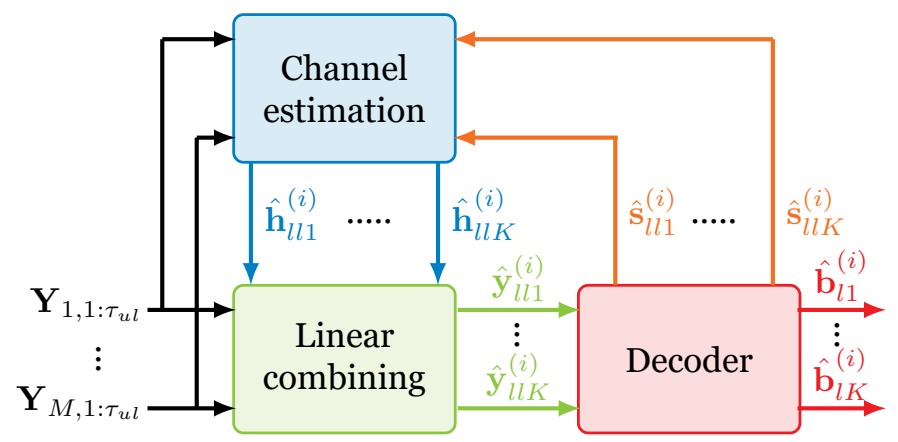

Figure 15: Block diagram of iterative receiver. The notation $\cdot^{(i)}$ is used to represent the values of variables at the $i^{\text {th }}$ iteration of the MICED algorithm.

The single-cell MMSE (S-MMSE) method seeks to balance interference suppression with signal amplification while only using CSI from UEs within the same cell as $\mathrm{UE}_{l k}$. By using the data observations in (89) and (90), the transmitted data symbols can be recovered through the decoding procedure.

Remark 1. Due to the unknown variations of the channel, interference and noise, the decoding procedure may fail to recover the desired information. In such cases, it is still possible to obtain data estimates that can be used as side information to improve the quality of the channel estimates and attempt to decode the data again. More importantly, if there are UEs whose data has been successfully decoded, perfect data estimates can be obtained to improve the decoding of unsuccessful UEs.

The principle of the MICED algorithm is to spread the interference effect between UEs. That is, to trade the main interfering sources in the channel estimation for reduced interference coming from all cells. The main source of interference in the channel estimation with RP is pilot contamination (see the first term in (83)), and this effect is reduced by collecting more channel observations from estimated data symbols. On the other hand, with $\mathrm{SP}$ the pilot contamination is reduced by having longer pilots and the main interference in the channel estimation is due to data symbols (see the second term in (84)), thus the aim of using data estimates in this case is to reduce the data intracell (same cell as $\mathrm{UE}_{l k}$ ) interference.

The MICED algorithm is done iteratively to improve data and channel estimates in each iteration as shown in Figure 15. Each iteration starts with the channel estimation, followed by linear combining, and finishing with the data decoding. The MICED algorithm ends when the maximum number of iterations is reached or the data from all UEs is successfully decoded. A 


\section{Algorithm 1 The MICED algorithm}

1: Initialize:

2: $\quad$ set $i=0$ and define $i_{\max } \in \mathbb{N}$

3: Pilot-based channel estimation for $\mathrm{UE}_{l k} \forall k \in\{1, \ldots, K\}$

4: $\quad$ calculate channel observations $\mathbf{z}_{l l k}^{(0)}$

5: $\quad$ calculate channel estimates $\hat{\mathbf{h}}_{l l k}^{(0)}$ $\triangleright$ use (79) with RP and (80) with SP

\section{6: Linear combining}

7: $\quad$ calculate combining vector $\mathbf{v}_{l k}^{(0)}$

8: $\quad$ calculate data observations $\hat{\mathbf{y}}_{l k}^{(0)}$

9: Data decoding for $\mathrm{UE}_{l k} \forall k \in\{1, \ldots, K\}$ $\triangleright$ use Lemma 6 with $\mathbf{z}_{l l k}^{(0)}$

10: decode data and extract data estimates $\hat{\mathbf{s}}_{l k}^{(0)}$

11: $\quad$ set $i=1$

12: $\quad$ while $i \leq i_{\max }$ or at least one UE has decoding errors do

13: $\quad$ Data-aided channel estimation for $\mathrm{UE}_{l k} \forall k \in\{1, \ldots, K\}$

14: $\quad$ calculate channel observations $\mathbf{z}_{l l k}^{(i)}=\mathbf{Y}_{l} \mathbf{u}_{l k}^{(i-1)^{*}}$ $\triangleright$ use (89) with RP and (90) with SP $\triangleright$ See Section 5 in Paper D for details $\mathbf{u}_{l k}^{(i)}=\left[\hat{\mathbf{X}}_{l}^{(i)}\left(\hat{\mathbf{X}}_{l}^{(i)}{ }^{H} \hat{\mathbf{X}}_{l}^{(i)}\right)^{-1}\right]_{k}$ is a de-spreading vector for $\mathrm{UE}_{l k}$ built from the signal estimates $\hat{\mathbf{X}}_{l}^{(i)}=\left[\hat{\mathbf{x}}_{l 1}^{(i)}, \ldots, \hat{\mathbf{x}}_{l K}^{(i)}\right] \in \mathbb{C}^{\tau_{c} \times K}$ that combine the knowledge from data estimates and pilots as

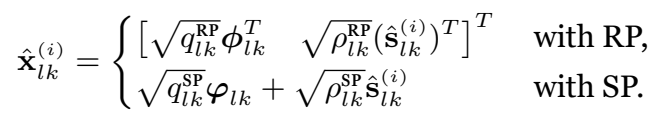

15: calculate channel estimates $\hat{\mathbf{h}}_{l l k}^{(i)}$

$\triangleright$ use Lemma 6 with $\mathbf{z}_{l l k}^{(i)}$

16: Linear combining

17: calculate combining vector $\mathbf{v}_{l k}^{(i)}$ $\triangleright$ use (91) with $\hat{\mathbf{h}}_{l l k}^{(i)}$

18: calculate data observation $\hat{\mathbf{y}}_{l k}^{(i)}$

$$
\begin{aligned}
& \hat{\mathbf{y}}_{l k}^{\mathrm{RP}(i)^{T}}=\mathbf{v}_{l k}^{\mathrm{RP}(i)}{ }^{H}\left(\left[\mathbf{Y}_{l}\right]_{\tau_{p}+1: \tau_{c}}-\sum_{\substack{k^{\prime}=1 \\
k^{\prime} \neq k}}^{K} \hat{\mathbf{h}}_{l l k^{\prime}}^{\mathrm{RP}(i)} \sqrt{\rho_{l k^{\prime}}^{\mathrm{RP}}} \hat{\mathbf{s}}_{l k^{\prime}}^{\mathrm{RP}(i-1)^{T}}\right), \\
& \hat{\mathbf{y}}_{l k}^{\mathrm{SP}(i)^{T}}=\mathbf{v}_{l k}^{\mathrm{SP}(i)}{ }^{H}\left(\mathbf{Y}_{l}-\sum_{k^{\prime}=1}^{K} \hat{\mathbf{h}}_{l l k^{\prime}}^{\mathrm{SP}(i)} \sqrt{q_{l k^{\prime}}^{\mathrm{SP}}} \boldsymbol{\varphi}_{l k^{\prime}}^{T}-\sum_{\substack{k^{\prime}=1 \\
k^{\prime} \neq k}}^{K} \hat{\mathbf{h}}_{l l k^{\prime}}^{\mathrm{SP}(i)} \sqrt{\rho_{l k^{\prime}}^{\mathrm{SP}}} \hat{\mathbf{s}}_{l k^{\prime}}^{\mathrm{SP}(i-1)^{T}}\right) .
\end{aligned}
$$

Apart form updating the combining vector, the data estimates and data-aided channel estimates are also used to subtract interference.

Data decoding for $\mathrm{UE}_{l k} \forall k \in\{1, \ldots, K\}$ page. 


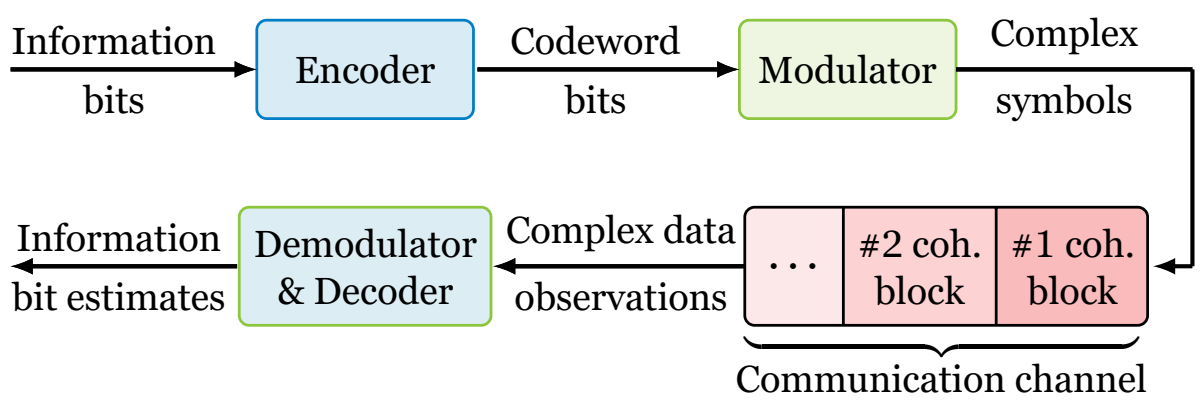

Figure 16: Basic communication model.

In practical communication systems, the transmitter produces information bits which form the desired information that needs to be successfully conveyed to the receiver. A basic communication model for this process is depicted in Figure 16. At the transmitter, the information bits are encoded into long bit sequences called codewords, which are then modulated into complex symbol and sent through the channel. At the receiver, the complex symbols are demodulated and decoded into bit estimates from which the information bits can be extracted. The process of encoding and decoding the information is known as channel coding and its purpose is to combat the errors introduced by the communication channel. This is done by increasing the number of bits to represent the desired information [95].

The achievable SE analysis conducted in this dissertation (see in Section 2.5) is based on the mutual information between the complex symbols and there is no need to specify a particular channel code that achieves said SE, it is only important to know that it exists. Thus, the details of selecting good channel codes, and implementing coding and decoding algorithms are deferred to the textbooks $[95,96]$.

To implement the MICED algorithm, it is necessary to extract data estimates from the output of the decoder. Thus, in this case it is necessary to include the effect of channel coding for an accurate evaluation of the possible benefits that the MICED algorithm can bring in practice. To obtain the data estimates, log-likelihood ratios (LLRs) per each bit in the codeword are computed based on the data observations obtained after linear combining (e.g., see (89), (90), (93) and (94)). These LLRs are then fed into the decoder which uses the redundant information in the channel code to attempt to correct errors in the received bits. At the decoder output, it is possible to obtain updated versions of the LLRs which can be used to find estimates of the transmitted complex data symbols. A more detailed description on how 
to obtain these data estimates is found in Paper D.

Remark 2. The decoder is able to determine if it has succeeded by checking if the received bits form a valid codeword. ${ }^{5}$ Thus, if the decoding procedure is successful for some UEs, their data estimates would be perfect and can be used to reduce interference. This in turn, can help UEs that have previously failed, to successfully decode their information. This is one of the main advantages of the MICED algorithm.

\section{Analysis of MICED with Gaussian data symbols}

To obtain insights into the mechanism used by the MICED algorithm to reduce interference, the data symbols are considered as i.i.d. $\mathbf{s}_{l k} \sim \mathcal{C N}\left(\mathbf{0}, \mathbf{I}_{\tau_{d}}\right)$ which allows for a tractable analysis of the channel estimation process. The correlation matrix of the channel observations on the $i^{\text {th }}$ iteration of the MICED algorithm is given by

$$
\begin{aligned}
\mathbf{\Psi}_{l l k}^{(i)}= & \mathbb{E}\left\{\mathbf{z}_{l l k}^{(i)} \mathbf{z}_{l l k}^{(i)}\right\}=\mathbf{R}_{l l k}+\underbrace{\sum_{k^{\prime}=1}^{K} \mathbf{R}_{l l k^{\prime}} \mathbb{E}\left\{\left|\mathbf{u}_{l k}^{(i)} \tilde{\mathbf{x}}_{l k^{\prime}}^{(i)}\right|^{2}\right\}}_{\text {Intracell interference }} \\
& +\underbrace{\sum_{\ell \in \Phi \backslash l} \sum_{k^{\prime}=1}^{K} \mathbf{R}_{l \ell k^{\prime}} \mathbb{E}\left\{\left|\mathbf{u}_{l k}^{(i)} \mathbf{x}_{\ell k^{\prime}}\right|^{2}\right\}}_{\text {Intercell interference }}+\underbrace{\sigma^{2} \mathbb{E}\left\{\left\|\mathbf{u}_{l k}^{(i)}\right\|^{2}\right\} \mathbf{I}_{M}}_{\text {Noise }}
\end{aligned}
$$

where $\tilde{\mathbf{x}}_{l k}^{(i)}=\mathbf{x}_{l k}-\hat{\mathbf{x}}_{l k}^{(i)}$ is the signal estimation error vector at the $i^{\text {th }}$ iteration. As it was shown in Section 2.4, to have ideal perfect channel estimation $\mathbf{\Psi}_{l l k}^{(i)} \rightarrow \mathbf{R}_{l l k}$ which means that the interference and noise terms in (95) should be as low as possible. The expectations in (95) involve inverse moments of non-central Wishart matrices which are difficult to compute. In Paper D closed-form expressions for lower bounds on these expectations are found to give detailed insights into the benefits of the MICED algorithm.

To offer a simple view on the main idea behind the MICED algorithm, let us go over the following theoretical example. ${ }^{6}$

\footnotetext{
${ }^{5}$ In practical channel codes, the number of valid codewords is much lower than all the possible combinations of bits forming a codeword. In addition, they are designed such that it is highly unlikely to mistake one codeword for another.

${ }^{6}$ Note that the Example 1 is a theoretical exercise to illustrate the principles of the MICED algorithm and should not be considered as a proof. For a rigorous analysis see Paper D.
} 
Example 1. Consider the matrices $\hat{\mathbf{X}}=\left[\hat{\mathbf{x}}_{1}, \ldots, \hat{\mathbf{x}}_{K}\right]$ with $\hat{\mathbf{x}}_{k} \sim \mathcal{C N}\left(\mathbf{0}, \sigma_{k}^{2} \mathbf{I}_{N}\right)$ and $\tilde{\mathbf{X}}=\left[\tilde{\mathbf{x}}_{1}, \ldots, \tilde{\mathbf{x}}_{K}\right]$ with $\tilde{\mathbf{x}}_{k} \sim \mathcal{C N}\left(\mathbf{0},\left(1-\sigma_{k}^{2}\right) \mathbf{I}_{N}\right)$ to have independent columns and to be independent of each other. The matrix $\hat{\mathbf{X}} \in \mathbb{C}^{N \times K}$ can be viewed a the signal estimates and $\tilde{\mathbf{X}} \in \mathbb{C}^{N \times K}$ as the signal estimation errors from UEs in a given cell. Define $\mathbf{u}_{k}=\left[\hat{\mathbf{X}}\left(\hat{\mathbf{X}}^{H} \hat{\mathbf{X}}\right)^{-1}\right]_{k}$ which can be interpreted as the MICED de-spreading vector (see Line 14 of Algorithm 1), then it holds that

$$
\begin{aligned}
\mathbb{E}\left\{\left|\mathbf{u}_{k}^{H} \tilde{\mathbf{x}}_{i}\right|^{2}\right\} & =\left(1-\sigma_{i}^{2}\right) \mathbb{E}\left\{\left\|\mathbf{u}_{k}\right\|^{2}\right\} \\
& =\left(1-\sigma_{i}^{2}\right) \mathbb{E}\left\{\left[\left(\hat{\mathbf{X}}^{H} \hat{\mathbf{X}}\right)^{-1}\right]_{k k}\right\}=\frac{1-\sigma_{i}^{2}}{\sigma_{k}^{2}(N-K)}
\end{aligned}
$$

where the fist equality follows from the independence between $\mathbf{u}_{k}$ and $\tilde{\mathbf{x}}_{i}$. The third equality in (96) follows from properties of the circularly symmetric complex Gaussian vectors [7, App. B].

Now consider the vector $\mathbf{y} \sim \mathcal{C N}\left(\mathbf{0}, \mathbf{I}_{N}\right)$ independent of $\hat{\mathbf{X}}$, which can be interpreted as the signal from a UE in a different cell or noise. Then, similarly as in (96), it holds that

$$
\mathbb{E}\left\{\left|\mathbf{u}_{k}^{H} \mathbf{y}\right|^{2}\right\}=\frac{1}{\sigma_{k}^{2}(N-K)}
$$

The term in (96) can be viewed as a simpler version of the first expectation in (95) corresponding to the intracell interference. Thus, in principle, as the data estimation quality increases, the intracell interference decreases. This is in line with the intuition provided in Remark 2 that accurate data estimates of UEs within a given cell can be useful to improve the data decoding of other UEs in the same cell. In the case of SP, this effect can reduce the intracell data interference which is the strongest source of interference in (84).

A simpler version of the second and third expectations in (95) is given in (97). Thus, in principle, as the number of samples in the signal estimates increases, the impact of intercell interference and noise decreases. In the case of RP, this is particularly useful to reduce the pilot contamination effect when the number of samples is large enough. Note that typical coherence block sizes in sub-6GHz frequency bands are above a couple hundred samples $[7,8]$.

In summary, with RP, by using the data estimates, the pilot contamination is traded for interference from all UEs that decreases with the quality of data estimates and number of data symbols, which in turn might be substantially smaller. Whereas with SP, the intracell interference from data symbols decreases with the quality of data estimates and the size of the coherence block. Thus, the purpose of utilizing data estimates to revise the channel 
Table 2: MICED simulation parameters.

\begin{tabular}{c|c} 
Parameter & Value \\
\hline System bandwidth & $B_{\mathrm{W}}=20[\mathrm{MHz}]$ \\
Maximum transmission power per UE & $10 \log _{10}\left(P_{\max } B_{\mathrm{W}}\right)=20[\mathrm{dBm}]$ \\
Proportion of pilot power with SP & $\Delta=0.3$ \\
Noise power & $10 \log _{10}\left(\sigma^{2} B_{\mathrm{W}}\right)=-94[\mathrm{dBm}]$ \\
Inter-BS distance & $0.15[\mathrm{~km}]$ \\
Pathloss exponent & $\alpha=3.76$ \\
Pathloss at $1 \mathrm{~km}$ & $\omega=148.1[\mathrm{~dB}]$ \\
Shadow fading std. deviation & $\sigma_{\mathrm{sh}}=10[\mathrm{~dB}]$ \\
Angular std. deviation & $\sigma_{\mathrm{ang}}=10^{\circ}$ \\
\hline
\end{tabular}

estimation is to trade a few terms that cause high interference with many terms that cause low interference.

To illustrate the possible SE gains of the MICED algorithm, Monte-Carlo simulations considering Gaussian distributed data symbols are presented in Figure 17. The SE is calculated based on the capacity analysis done in Section 2.5. The simulation setup is based on a hexagonal cell grid with $K$ UEs uniformly distributed in each cell. The LSF is modeled as in Section 3.1.2 while including shadowing effects, that is, $\beta_{l l k}=\omega^{-1} d_{l l k}^{-\alpha} \digamma_{l l k}$ where $\digamma_{l l k}$ accounts for the shadow fading and is defined as $10 \log _{10}\left(\digamma_{l l k}\right) \sim \mathcal{N}\left(0, \sigma_{\mathrm{sh}}^{2}\right)$. The spatial correlation matrices are computed based on the Gaussian local scattering model with angular standard deviation $\sigma_{\text {ang }}$ defined in Section 2.3.2. Statistical channel inversion power control is considered such that $\varrho_{l k}=\min \left\{\varrho / \beta_{l l k}, P_{\max }\right\}$ where $\varrho$ sets the desired SNR level and $P_{\max }$ is the maximum transmission energy per symbol for each UE. In the case of SP, the proportion between pilot and data power is fixed as $\Delta_{l k}=\Delta$ and it is selected to maximize the average SE per UE with pilot-based channel estimation only. A summary of the main simulation parameters are given in Table 2.

Figure 17 shows the SE per UE versus $\tau_{c}$ and the sum SE per cell as a function of $K$, with MR processing (more results are included in Paper D). It can be seen that the MICED algorithm increases the SE in all cases. By comparing the SE with RP and SP using the MICED algorithm, Figure 17 shows that RP provides higher SE than SP when $\tau_{c}$ is high compared to $K$, whereas, the opposite happens when $\tau_{c}$ is comparable to $K$.

Remark 3. The cross point between the SE with RP and SP with respect to $\tau_{c}$ (see in Figure 17a), and K (see Figure 17b) indicates that the MICED algorithm with SP also has the possibility to utilize more aggressive spatial 


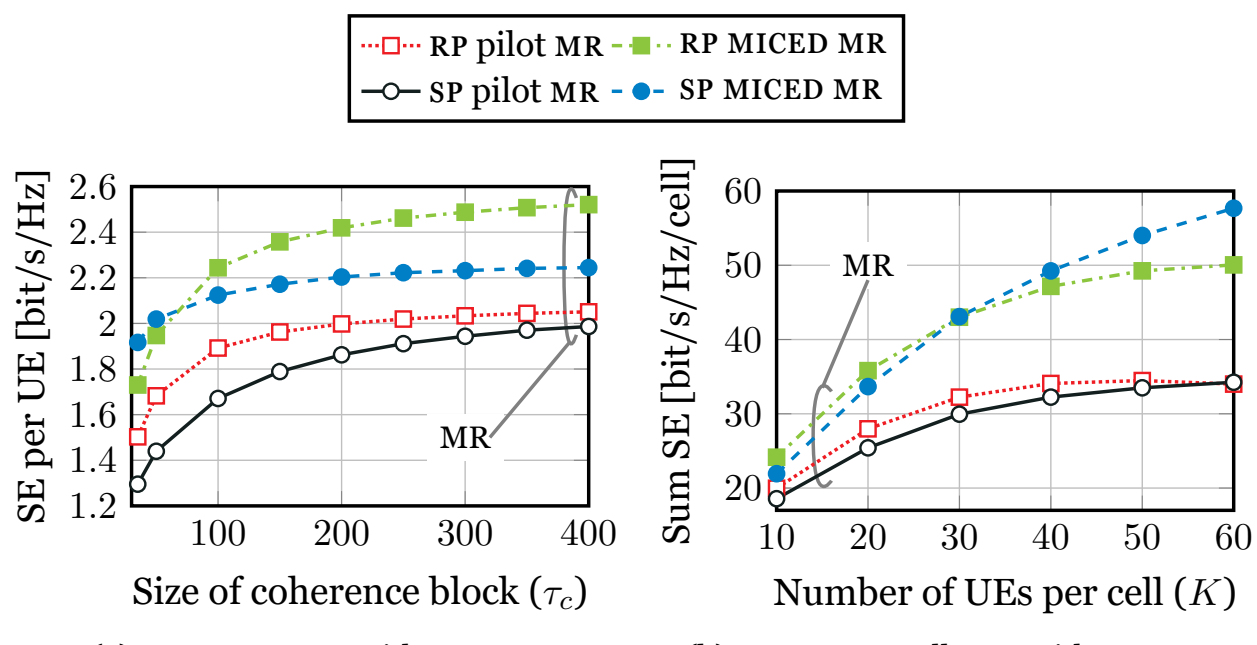

(a) SE per UE vs $\tau_{c}$ with $K=10$.

(b) Sum SE per cell vs $K$ with $\tau_{c}=190$.

Figure 17: SE per UE versus $\tau_{c}$, and sum SE per cell versus $K$; for $\varrho=\sigma^{2}(\mathrm{SNR}=0 \mathrm{~dB})$ and $M=100$. The data estimation quality is selected such that $\sigma_{l k}^{(i)^{2}}=0.6 \forall k \in\{1, \ldots, K\}$.

multiplexing that not only increases SE but also facilitates the implementation of machine type communication systems where many UEs need to be served.

\section{Analysis of MICED with finite alphabet data symbols}

To assess the benefits that the MICED algorithm can bring in practical implementations, a finite alphabet modulation is assumed to map the encoded bits into complex data symbols. Since typical codewords are made up of long sequences of bits, the resulting number of modulated symbols tends to span several coherence blocks, as illustrated in Figure 18. Moreover, the pilot symbols are inserted into each coherence block along with the modulated data symbols resulting in different number of coherence blocks that contain a full codeword with RP or SP.

The achievable SE at the $i^{\text {th }}$ iteration is obtained from the mutual information between the input bits, and the soft data symbol estimates at the output of the decoder. Let $N_{b}$ be the number of bits per complex modulated data symbol and $R_{c d}$ the code rate of the channel code used. To obtain the data estimates, the receiver needs the data observations from all complex symbols that contain all bits in a given codeword. Thus, let $N_{\mathrm{CW}}$ be the number of bits that make a full codeword, and denote by $\overline{\mathbf{y}}_{l k} \in \mathbb{C}^{N_{\mathrm{cw}} / N_{b}}$ the 
RP: $\square$ Pilot $\square$ Data

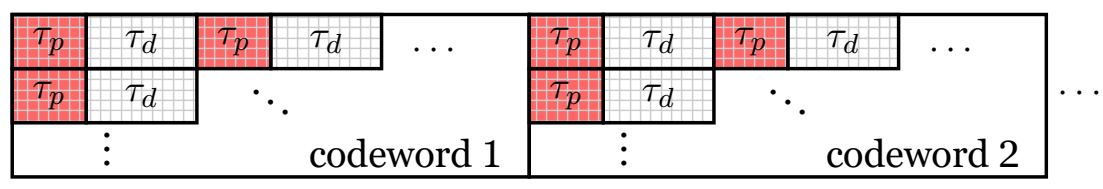

SP: $\square$ Pilot + data

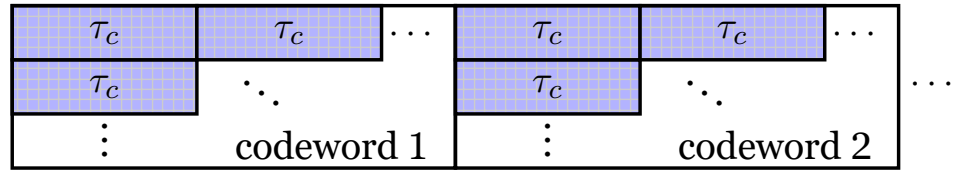

Figure 18: Time-frequency resource allocation with RP and SP over codewords of equal length.

observations of the corresponding complex data symbols obtained by staking several instances of (89) or (93) with RP, and, (90) or (94) with SP. The number of information bits encoded into a codeword is denoted as $N_{\mathrm{ENC}}$, and these encoded bits are staked into the vector $\mathbf{b}_{l k}$ such that $\left[\mathbf{b}_{l k}\right]_{m} \in\{0,1\}$ for $m \in\left\{1, \ldots, N_{\mathrm{ENC}}\right\}$. Assuming that the bits at the output of the decoder are independent, then the achievable SE is

$\mathrm{SE}_{l k}=\frac{\tau_{d} N_{b} R_{c d}}{\tau_{c} N_{\mathrm{ENC}}} \sum_{m=1}^{N_{\mathrm{ENC}}}\left(1+\mathbb{E}\left\{\sum_{d=0}^{1} \operatorname{Pr}\left(\left[\mathbf{b}_{l k}\right]_{m}=d \mid \overline{\mathbf{y}}_{l k}^{(i)}\right) \log _{2}\left(\operatorname{Pr}\left(\left[\mathbf{b}_{l k}\right]_{m}=d \mid \overline{\mathbf{y}}_{l k}^{(i)}\right)\right)\right\}\right)$

where the conditional probabilities in (98) are obtained based on the LLRs at the output of the decoder, see Paper D for details. Note that the achievable SE in (98) accounts the overhead from coding and using dedicated pilot symbols in the case of RP. It is worth mentioning that the MICED algorithm does not depend on the channel code being used, and thus, it can be implemented with any state of the art decoder.

To illustrate the gains of the MICED algorithm in a practical system, the same simulation setup as the one in the analysis with Gaussian distributed symbols is used. However, in this case considering LDPC codes and QPSK modulation. The choice of parity check matrix for the LDPC code is done following the new radio (NR) 3GPP specifications [97] with code rate $R_{c d}=3 / 4$ and a codeword length of 3888 bits. The results of implementing the MICED algorithm with RP assume pilot reuse one $\left(\tau_{p}=K\right)$. In the case of SP, the pilot reuse is the closest to $\tau_{c} / K$ that is allowed in an hexagonal grid. To establish a benchmark, the performance of standard pilot-based channel 


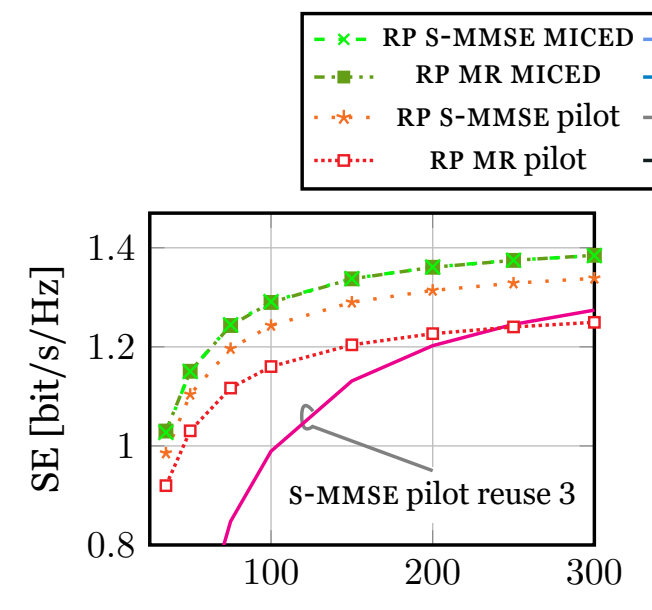

Size of coherence block $\left(\tau_{c}\right)$

(a) Achievable SE vs $\tau_{c}$ with RP.

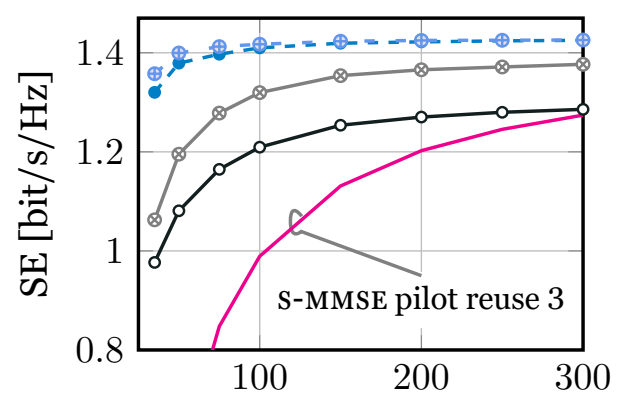

Size of coherence block $\left(\tau_{c}\right)$

(b) Achievable SE vs $\tau_{c}$ with SP.

Figure 19: Achievable SE versus coherence block size with $i=8$, $M=100, K=10, \varrho=\sigma^{2}(\mathrm{SNR}=0 \mathrm{~dB})$, and $R_{c d}=3 / 4$. The SE with RP, pilot-based channel estimation, S-MMSE combining, and pilot reuse 3 is included as a benchmark.

estimation with RP, S-MMSE combining and pilot reuse 3 is included.

Figure 19 shows the achievable SE as a function of the coherence block size. It can be seen that the MICED algorithm increases the SE compared to pilot-based channel estimation with both RP and SP. The highest SE is achieved by SP since there is not pilot overhead and the MICED algorithm reduces the interference from data symbols. In particular, the highest SE gains with SP and the MICED algorithm are found for low values of $\tau_{c}$.

Remark 4. The alternative SP design combined with the proposed MICED algorithm is able to increase the SE compared to the Massive MIMO baseline. The highest SE gain is found in cases with short coherence block size which correspond to high mobility or low carrier frequency. In addition, this alternative design can support aggressive spatial multiplexing which, in turn, enables other services like machine type communications. 


\subsection{Hardware Impairments Optimization}

Due to the large number of BS antennas deployed in Massive MIMO, it is desirable to relax the quality of the RF chains to limit the implementation costs and power consumption. Thus, the design of the hardware needs to balance high performance with low complexity. In this section, a single cell MU-MIMO system that deploys $M$ BS antennas serving $K$ UEs is considered. To evaluate the impact of relaxing the quality of RF chains into the SE, power consumption and EE; the analysis in this section introduces a parameter called the level of hardware impairments (HWIs). Furthermore, this level of HWIs is selected to represent the ADC bit resolution within the RF chains. The Massive MIMO baseline, considers equal ADC bit resolution across the BS antenna elements. In contrast, the alternative Massive MIMO design called mixed-ADCs, allows the ADC bit resolution of each BS antenna element to be different. Thus, two main research questions are investigated:

Question 1), analyzed in Paper E: What is the optimal tradeoff between the number of BS antennas and the level of HWIs considering SE and power consumption?

Question 2), analyzed in Papers F and G: What is the optimal allocation of HWIs across the BS antennas in terms of maximal SE and minimum power consumption? Does the alternative design with mixedADCs offer higher SE or EE compared to the Massive MIMO baseline design?

The rest of this section is organized as follows: In Section 3.2.1, the models for HWIs used in this dissertation are introduced and motivated. Section 3.2.2 gives the answer to question 1) by evaluating the tradeoff between number of BS antennas and level of HWIs. In Section 3.2.3, the first part of the answer to question 2) is given by assuming i.i.d. Rayleigh fading. Here, majorization theory is used to assess the order presenting properties of $\mathrm{SE}$ and power consumption with respect to the ADC bit allocation per antenna. Section 3.2.4, gives the second (and final) part of the answer to question 2), where correlation and LSF variations between the UL channels towards the BS are considered. In this case, optimization problems for minimum pilot distortion, maximum SINR or minimum power consumption are formulated to evaluate the impact of $\mathrm{ADC}$ allocation on the SE and EE.

\subsubsection{Parametrized HWIs Model}

In this dissertation, a parametrized model is adopted to encompass the effects of non-coherent distortion caused by the HWIs. For the first question, 
the effect of the HWIs at the BS is modeled by a reduction of the received signal energy per symbol of $1-\epsilon^{2}$ which is turned into distortion defined as $\mathbf{e} \in \mathbb{C}^{M \times 1}$. This assumption is made to have energy conservation after the signal is affected by HWIs. The parameter $\epsilon \in[0,1]$ refers to the level of HWIs such that $\epsilon=0$ indicates perfect hardware and as $\epsilon$ increases, the hardware quality decreases. The received signal at the BS is

$$
\mathbf{y}=\sqrt{1-\epsilon^{2}}\left(\sum_{i=1}^{K} \mathbf{h}_{i} x_{i}+\mathbf{n}\right)+\mathbf{e}
$$

where $\mathrm{UE}_{i}$ transmits the signal $x_{i}$ (corresponding to pilots or data) and the noise is $\mathbf{n} \sim \mathcal{C N}\left(\mathbf{0}, \sigma^{2} \mathbf{I}_{M}\right)$ with average energy per symbol given by $\sigma^{2}$. The distortion is defined as

$$
\mathbf{e}=\sqrt{\epsilon^{2}\left(\sum_{i=1}^{K} \mathbb{E}\left\{\left|x_{i}\right|^{2}\right\} \mathbf{D}_{\left|\mathbf{h}_{i}\right|^{2}}+\mathbf{I}_{M} \sigma^{2}\right) \mathbf{r}}
$$

where $\mathbf{D}_{\left|\mathbf{h}_{i}\right|^{2}}=\operatorname{diag}\left(\left|\left[\mathbf{h}_{i}\right]_{1}\right|^{2}, \ldots,\left|\left[\mathbf{h}_{i}\right]_{M}\right|^{2}\right) \forall i \in\{1, \ldots, K\}$ and the randomness of the distortion is modeled by $\mathbf{r} \sim \mathcal{C N}\left(\mathbf{0}, \mathbf{I}_{M}\right)$. The term enclosed in the squared root in (100) accounts for the missing energy per symbol that has been taken from the received signal by the term $1-\epsilon^{2}$ in (99).

The model in (99) is based on Bussgang's Theorem [98]. This theorem states that the cross-correlation between two stationary random processes that are jointly Gaussian, when one of them is passed through a memoryless non-linear deterministic function, is a scaled version of the cross-correlation between the input processes. The following description shows how the Bussgang's Theorem can be used to decompose the received signal affected by hardware distortions into a linear function of the input signal plus a distortion term that is uncorrelated to the input (for more details see [99]).

\section{Bussgang's decomposition to model HWIs}

The received signal without noise at the BS, that is affected by hardware impairments, is given by ${ }^{7}$

$$
\mathbf{y}=\mathbf{f}_{\mathrm{HW}}(\mathbf{H s})
$$

where $\mathbf{H}=\left[\mathbf{h}_{1}, \ldots, \mathbf{h}_{K}\right] \in \mathbb{C}^{M \times K}$ is the channel matrix (including the scaling for transmission energy per symbol) and $\mathbf{s} \in \mathbb{C}^{K}$ is the vector

\footnotetext{
${ }^{7}$ Note that the noise is not included for ease of notation. However, if the noise is included and assumed to be circularly symmetric complex Gaussian distributed, the analysis follows the same steps.
} 
with data symbols transmitted by the $K$ UEs in one arbitrary sample of the coherence block. The symbols are assumed to be zero-mean, independent, and to have unit energy, such that, $\mathbb{E}\left\{\mathbf{s s}^{H}\right\}=\mathbf{I}_{K}$. The HWIs that affect the received signal at the $m^{\text {th }}$ BS antenna element, are modeled by a memoryless, arbitrary and deterministic function denoted as $f_{\mathrm{HW}_{m}}(\cdot): \mathbb{C} \rightarrow \mathbb{C}$ such that $\left[\mathbf{f}_{\mathrm{HW}}(\mathbf{H s})\right]_{m}=f_{\mathrm{HW}_{m}}\left([\mathbf{H s}]_{m}\right)$ for $m \in\{1, \ldots, M\}$. This function can be used to represent many nonlinear effects in the RF chain, such as, clipping, quantization, phase rotations, among others.

By computing the LMMSE estimate of $\mathbf{y}$ based on the observation $\mathbf{H s ,}$ assuming that $\mathbf{H}$ is known, the distorted signal can be expressed as

$$
\mathbf{y}=\underbrace{\mathbb{E}\left\{\mathbf{y}(\mathbf{H s})^{H} \mid \mathbf{H}\right\}\left(\mathbf{H H}^{H}\right)^{\dagger}(\mathbf{H s})}_{=\hat{\mathbf{y}} \text { (i.e., LMMSE est. of } y \text { ) }}+\mathbf{n}_{\mathrm{HW}}
$$

where $\mathbf{n}_{\mathrm{HW}}=\mathbf{f}_{\mathrm{HW}}(\mathbf{H s})-\hat{\mathbf{y}}$ can be defined as the estimation error that is uncorrelated to Hs. The notation $(\cdot)^{\dagger}$ stands for the pseudo inverse (or Moore-Penrose inverse) since $\mathbf{H H}^{H}$ is rank deficient for $M>K$, which is the case in Massive MIMO. The result in (102) can be used to model the HWIs effect as a linear function of the input signal Hs plus a noise that is uncorrelated to Hs. This model does not assume a particular distribution for the data symbols and can be used to model the effect of HWIs in finite-alphabet (e.g., QPSK, QAM, etc.) or continuous distributions.

Since in the achievable SE analysis, such as in Section 2.5, the data symbols are defined as Gaussian distributed, that is, $\mathbf{s} \sim \mathcal{C N}\left(\mathbf{0}, \mathbf{I}_{K}\right)$, the Bussgang Theorem [98] can be used to simplify the model in (102). The following lemma introduces an adapted version of the Bussgang Theorem for complex-valued discrete variables, see [99] for more details.

Lemma 7. Consider two jointly circularly symmetric complex Gaussian variables $x$ and $y$. For any deterministic function $f_{\mathrm{HW}}(\cdot): \mathbb{C} \rightarrow \mathbb{C}$, it holds that

$$
\mathbb{E}\left\{f_{\mathrm{HW}}(x) y^{*}\right\}=\mathbb{E}\left\{f_{\mathrm{HW}}(x) x^{*}\right\} \frac{\mathbb{E}\left\{x y^{*}\right\}}{\mathbb{E}\left\{|x|^{2}\right\}}
$$

Proof. It follows from defining $y$ in terms of a LMMSE estimate from the observation $x$ and an uncorrelated noise denoted as $n_{\mathrm{hw}}$. This means that, $y=\frac{\mathbb{E}\left\{y x^{*}\right\}}{\mathbb{E}\left\{|x|^{2}\right\}} x+n_{\mathrm{hw}}$ where $n_{\mathrm{HW}}=y-\frac{\mathbb{E}\left\{y x^{*}\right\}}{\mathbb{E}\left\{|x|^{2}\right\}} x$. Since $x$ 
and $n_{\mathrm{HW}}$ are uncorrelated and jointly Gaussian distributed, they are also independent. Finally, by introducing $y=\frac{\mathbb{E}\left\{y x^{*}\right\}}{\mathbb{E}\left\{|x|^{2}\right\}} x+n_{\mathrm{hw}}$ in the left-hand-side of (103), and realizing that $\mathbb{E}\left\{f_{\mathrm{HW}}(x) n_{\mathrm{HW}}^{*}\right\}=0$, since $n_{\mathrm{HW}}$ is zero-mean and independent of $x$, the right-hand-side of (103) is obtained.

By using Lemma 7 the received signal can be simplified as

$$
\mathbf{y}=\mathbf{D}_{\mathrm{Buss}} \mathbf{H s}+\mathbf{n}_{\mathrm{HW}}
$$

where $\mathbf{D}_{\text {Buss }}$ is a diagonal matrix given by

$$
\left[\mathbf{D}_{\mathrm{BUSs}}\right]_{m m}=\frac{\mathbb{E}\left\{f_{\mathrm{HW}_{m}}\left([\mathbf{H s}]_{m}\right)[\mathbf{H s}]_{m}^{*} \mid \mathbf{H}\right\}}{\left[\mathbf{H} \mathbf{H}^{H}\right]_{m}} \quad \forall m \in\{1, \ldots, M\}
$$

and the distortion is given by $\mathbf{n}_{\mathrm{HW}}=\mathbf{f}_{\mathrm{HW}}(\mathbf{H s})-\mathbf{D}_{\mathrm{BUss}} \mathbf{H s}$ which is uncorrelated to Hs. The correlation matrix of the distortion conditioned on the channel realizations is given by

$$
\mathbb{E}\left\{\mathbf{n}_{\mathrm{HW}} \mathbf{n}_{\mathrm{HW}}^{H} \mid \mathbf{H}\right\}=\mathbb{E}\left\{\mathbf{f}_{\mathrm{HW}}(\mathbf{H s}) \mathbf{f}_{\mathrm{HW}}(\mathbf{H s})^{H} \mid \mathbf{H}\right\}-\mathbf{D}_{\text {BUss }} \mathbf{H} \mathbf{H}^{H} \mathbf{D}_{\text {BUSs }}^{H} .
$$

To motivate the parametrized model in (99) and (100) and assess its limitations, let us compare it with the Bussgang's decomposition in (105). First, the level of HWIs is assumed to be the same for all BS antennas which means that the HWI function in (101) is the same for each element on the BS antenna array. Second, the term $1-\epsilon^{2}$ is related to the correlation between the signal before and after it has passed through the HWI function. Note that if perfect hardware is assumed then $f_{\mathrm{HW}}\left([\mathbf{H s}]_{m}\right)=[\mathbf{H s}]_{m}$ and $\mathbf{D}_{\mathrm{Buss}}=\mathbf{I}_{M}$ (see (105)) which coincides with $\epsilon=0$. Whereas, if the HWI function distorts the signal to such a degree that it becomes uncorrelated to the input signal, then this corresponds to the worst case of $\epsilon=1$. Third, notice that the hardware distortion $\mathbf{n}_{\mathrm{HW}}$ in (104) accounts for the missing energy taken by $\mathbf{D}_{\text {Buss }}$ which is the same principle used to define e in (100). Fourth, the correlation matrix of the hardware distortion in (106) is in general non-diagonal, let alone for the fact that $\mathbf{H H}^{H}$ is rank deficient for $M>K$. This effect has been neglected in (100) and is one of the limitations of this parametrized model. However, this correlation has been shown to have limited impact when there are many UEs [99], particularly when it comes to quantization, and it is a common practice to neglect it $[36,37,44]$. Finally, since the HWI function is deterministic, the hardware distortion $\mathbf{n}_{\mathrm{HW}}$ is statistically dependent of the input signal $\mathbf{H s}$ which is an effect that is also neglected in the parametrized 
model (100) since $\mathbf{r}$ is assumed independent of the data symbols. This latter assumption enables a tractable analysis of the hardware distortion and yields a closed-form expression for the SE under MR combining. This SE expression can be used in optimization problems to obtain key insights into the hardware design. In later analysis, the SE expressions obtained with a simplified version of the parametrized model in (99) and (100), will be compared to the SE of an exact quantization model.

The parametrized model in (99) and (100) is commonly used for evaluating the overall system performance of the network under the effect of residual HWIs [32, 100]. Note that in this model, the distortion noise depends on the instantaneous realizations of the channel which gives good insights into the effects introduced by imperfect CSI with added distortion. The reason is that the HWIs distort the received signals from pilot and data symbols which are affected by the same channel realization. Thus, when performing linear combining, the hardware distortions in the channel estimates and the received data signal, would be statistically dependent through the channel realizations.

We stress that the HWIs model in (99) and (100) is an approximated model and it should be used with caution. The aim of this system model is to find insights into the design of the HWIs level based on an achievable SE analysis as the one in Section 2.5. It should be noted that the parametrized model does not constitute an accurate representation of the physical properties of the hardware and it should not be used with this intend. For example, this model cannot be used to describe the nonlinear effects associated with out-of-band interference.

For the second research question stated in Section 3.2, the level of HWIs is redefined as $\varepsilon^{2}=\epsilon^{2} /\left(1-\epsilon^{2}\right)$ to have a more compact notation. The same model as in (99) and (100) is used with the difference that each antenna element has an individual level of HWIs that is enclosed by the matrix $\mathbf{D}_{\varepsilon}=\operatorname{diag}\left(\varepsilon_{1}^{2}, \ldots, \varepsilon_{M}^{2}\right)$. The received signal at the BS is

$$
\mathbf{y}=\sum_{i=1}^{K} \mathbf{h}_{i} x_{i}+\mathbf{n}+\mathbf{e}
$$

and the distortion noise is defined as

$$
\mathbf{e}= \begin{cases}\mathbf{D}_{\varepsilon}^{\frac{1}{2}}\left(\sum_{i=1}^{K} \mathbb{E}\left\{\left|x_{i}\right|^{2}\right\} \mathbf{D}_{\left|\mathbf{h}_{i}\right|^{2}}+\sigma^{2} \mathbf{I}_{M}\right)^{\frac{1}{2}} \mathbf{r} & \text { in Paper F, } \\ \mathbf{D}_{\varepsilon}^{\frac{1}{2}}\left(\sum_{i=1}^{K} \mathbb{E}\left\{\left|x_{i}\right|^{2}\right\} \mathbf{D}_{\left|\mathbf{h}_{i}\right|^{2}}\right)^{\frac{1}{2}} \mathbf{r} & \text { in Paper G. }\end{cases}
$$

The model used in Paper F includes the average noise energy in the hardware distortion while Paper G does not. This difference is only done to have a 
more compact representation of the HWI distortion in Paper G that makes the analytical expressions easier to interpret. The SE expressions obtained with the models in (107) and (108), are compared to the SE obtained with an exact quantization model in Sections 3.2.3 and 3.2.4.

\subsubsection{Optimal Tradeoff between HWIs and BS Antennas}

In what follows, the main analytical and numerical results needed to answer the first research question are explained along with the main insights and the answer to the question. Considering the system model in (99) and (100), a lower bound on the sum capacity based on the analysis in Section 2.5, assuming LMMSE channel estimation and MR combining is given by ${ }^{8}$

$$
\begin{aligned}
& \mathrm{R}_{\mathrm{SUM}}(\epsilon, M)=\left(1-\frac{\tau_{p}}{\tau_{u l}}\right) \sum_{k=1}^{K} \log _{2}\left(1+\mathrm{SINR}_{k}\right) \\
& \operatorname{SINR}_{k}=\left(1-\epsilon^{2}\right)^{2} M / \underbrace{\left(\epsilon^{2}\left(1-\epsilon^{2}\right)+\frac{2 \epsilon^{2}\left(1-\epsilon^{2}\right)\left(\frac{\epsilon^{2}}{\tau_{p}} \sum_{i=1}^{K} \frac{\beta_{i}^{2} \rho_{i}^{2}}{\beta_{k}^{2} \rho_{k}^{2}}+2\left(1-\epsilon^{2}\right)\right)}{\tau_{p}\left(\left(1-\epsilon^{2}\right)+\frac{\epsilon^{2}}{\tau_{p}} \sum_{i=1}^{K} \frac{\beta_{i} \rho_{i}}{\beta_{k} \rho_{k}}+\frac{\sigma^{2}}{\tau_{p} \beta_{k} \rho_{k}}\right)^{2}}\right.}_{\text {Hardware distortion }} \\
& +\underbrace{\left(\left(1-\epsilon^{2}\right)+\frac{\epsilon^{2}}{\tau_{p}} \sum_{i=1}^{K} \frac{\beta_{i} \rho_{i}}{\beta_{k} \rho_{k}}+\frac{\sigma^{2}}{\tau_{p} \beta_{k} \rho_{k}}\right)}_{\text {Loss in the coherent gain from imperfect CSI }} \underbrace{\left(\sum_{i=1}^{K} \frac{\beta_{i} \rho_{i}}{\beta_{k} \rho_{k}}+\frac{\sigma^{2}}{\rho_{k} \beta_{k}}\right)}_{\text {Non-coherent interference }}),
\end{aligned}
$$

where the transmission energy per symbol of pilot and data from $\mathrm{UE}_{i}$ is $\rho_{i}$. It is worth mentioning that, since the distortion depends on the exact realizations of the channel (see (100)) the observations used for channel estimation are non-Gaussian, and therefore, LMMSE channel estimation is used. To facilitate later analysis, $\mathrm{R}_{\mathrm{SUM}}(\epsilon, M)$, is defined as a function of $\epsilon$ and $M$.

This work assumes that the ADCs are the main source of power consumption that relates to the level of HWIs and the following relationship is established, $\epsilon=\zeta 2^{-b}$, where $1<\zeta<2$ is a constant related to the saturation level of the ADC $[33,101]$. The power consumption is modeled as in Section 2.6 where $\mathrm{P}_{\mathrm{CD}}=0$ in order to focus on the power consumed by the circuitry that is related to the HWIs. Then the power consumption is also

\footnotetext{
${ }^{8}$ Notice that this result assumes the same power allocation for pilot and data symbols.
} 
defined as a function of $\epsilon$ and $M$ given by (see (33), (38), and (39))

$$
\mathrm{P}(\epsilon, M)=\mathrm{C}_{0}+\mathrm{D}_{0} M+\mathrm{D}_{1} 2 M \zeta^{\varphi} \epsilon^{-\varphi}+\frac{B_{\mathrm{W}}}{L} 2 M K \log _{2}\left(\frac{\zeta}{\epsilon}\right)
$$

where $\mathrm{C}_{0}$ represents all terms that are independent of $\epsilon$ and $M .{ }^{9}$ To find the optimal tradeoff between $\epsilon$ and $M$ in terms of $\mathrm{R}_{\text {SuM }}(\epsilon, M)$ and $\mathrm{P}(\epsilon, M)$, the following optimization problem is formulated:

$$
\begin{aligned}
\underset{\epsilon \in[0,1], M \in \mathbb{N}}{\operatorname{maximize}} & \mathrm{R}_{\mathrm{SUM}}(\epsilon, M), \\
\text { subject to } & \mathrm{P}(\epsilon, M) \leq \gamma,
\end{aligned}
$$

where $\gamma[\mathrm{W}]$ is the power constraint at the BS. The optimal tradeoff that solves the optimization in (112) is given in the following lemma.

Lemma 8. Assuming that the optimization problem (112) is feasible, then the maximum $\mathrm{R}_{\mathrm{Sum}}$ is found when $\mathrm{P}(\epsilon, M)=\gamma$, thus the optimal values of $\epsilon$ and $M$ are related as

$$
M=\frac{\gamma-\mathrm{C}_{0}}{\mathrm{D}_{0}+2 \mathrm{D}_{1} \zeta^{\varphi} \epsilon^{-\varphi}+\frac{B_{\mathrm{W}}}{L} 2 K \log _{2}\left(\frac{\zeta}{\epsilon}\right)} .
$$

Proof. It follows from the monotonicity of the functions $\mathrm{R}_{\mathrm{SUM}}(\epsilon, M)$ and $\mathrm{P}(\epsilon, M)$. A more detailed proof can be found in Paper E.

Numerical results are presented to illustrate the optimal tradeoff between $\epsilon$ and $M$, along with the SE and EE performance. Statistical channel inversion power control is used for pilot and data symbols so that $\rho_{k}=\varrho_{u l} / \beta_{k}$ where $\beta_{k}=\omega^{-1} d_{k}^{-\alpha}$ is the LSF coefficient defined as in Section 2.6 (see Paper E for details). The UEs are distributed uniformly at random in a circular cell with the BS at the center. The main parameters used in this study are depicted in Table 1 shown in Section 2.6. Figure 20 illustrates the sum $\mathrm{SE}$ versus the level of HWIs and the number of BS antennas. The optimal condition in (113) is depicted by the dark marks for $\gamma=\{22,26,30\}$ [W] and the corresponding maximum sum SE is found at $M=\{87,126,164\}$ and $\epsilon=\{0.055,0.056,0.056\}$ which corresponds to an $\mathrm{ADC}$ resolution of $b \approx 4$ or 5 bits. By varying the power constraint $\gamma$, maximal EE can be achieved which is shown in Figure 21.

\footnotetext{
${ }^{9}$ The last term in (111) accounts for the channel estimation and linear processing, and in this analysis it is considered to be dependent on the ADC bit resolution for completeness. In practice, however, the operations done after quantization are carried out in dedicated hardware which has higher precision. Thus, the analysis in Sections 2.6 and 3.2.4 calculate the power consumption of channel estimation and linear processing based on [flops/W].
} 


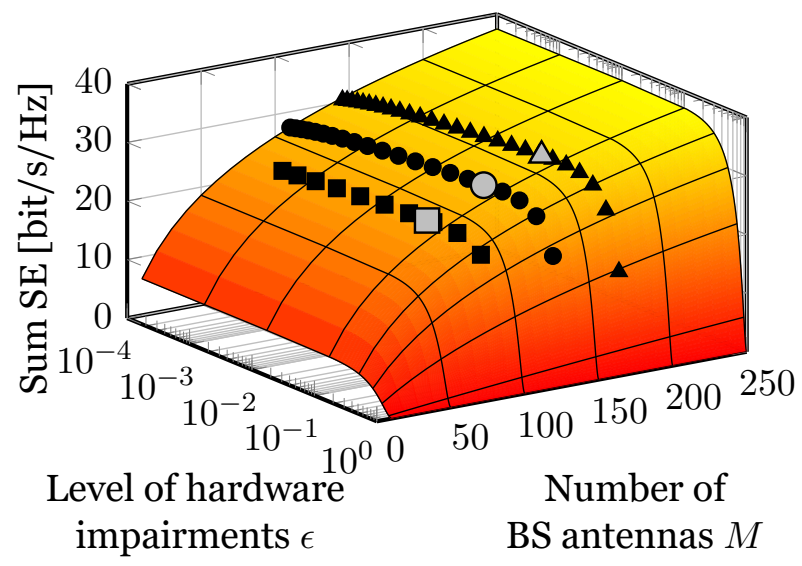

Figure 20: Sum SE versus $M$ and $\epsilon$ for $K=10$, and $10 \log _{10}\left(\frac{\varrho_{u l}}{\sigma^{2}}\right)=-5[\mathrm{~dB}]$. The dark square, circle and triangle points represent the curves where $\mathrm{P}(\epsilon, M)=\gamma$ for $\gamma=\{22,26,30\}[\mathrm{W}]$ respectively, and the lighter points are the corresponding maximum SE.

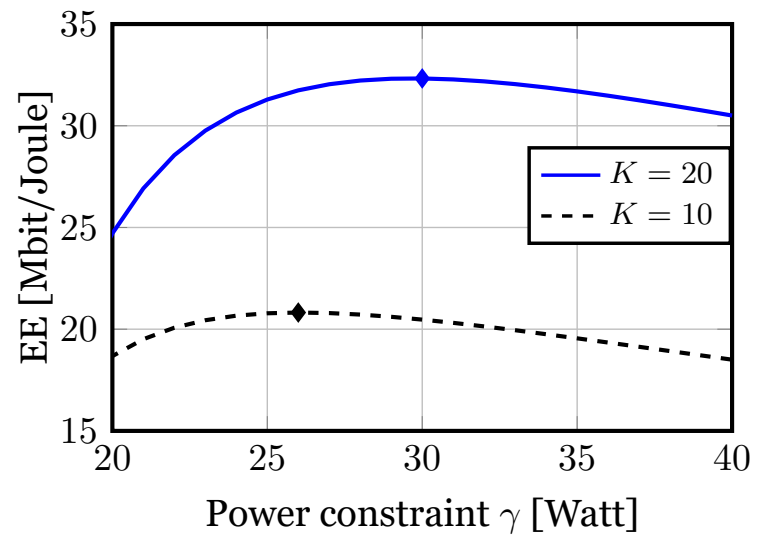

Figure 21: $\mathrm{EE}$ versus $\gamma$ for $K=\{10,20\}$, and $10 \log _{10}\left(\frac{\underline{\varrho}_{u l}}{\sigma^{2}}\right)=-5[\mathrm{~dB}]$. The marks represent the maximum EE located at $\gamma^{*}=\{26,30\}$ [W], which corresponds to $M^{*}=\{126,133\}$ and $\epsilon^{*}=\{0.056,0.0795\}$ respectively.

From (113) in Lemma 8, it can be seem that as $\epsilon$ becomes smaller (i.e., the $\mathrm{ADC}$ bit resolution increases), the number of optimal BS antennas decreases which, in turn, reduces the SE as shown in Figure 20. On the other hand, when $\epsilon$ grows too much such that it approaches $\zeta / 2$ (i.e., the ADC bit resolution 
equals one), more BS antennas are allowed but the sum SE decreases rapidly as shown in Figure 20. In summary, if the ADC bit resolution is too high, too much power is consumed and if the ADC bit resolution is one, the loss in SE is too high. Thus, neither one bit or infinite bit $\mathrm{ADC}$ resolution is optimum to balance SE and power consumption.

Answer 1) The optimal tradeoff between the number of BS antennas and the level of HWIs is given by (113) as a result of solving the optimization problem in (112). Based on numerical results, the optimal solution that maximizes SE and EE lies in a Massive MIMO setup with hundreds of antennas, and $b \approx 4$ or 5 bits of $\mathrm{ADC}$ resolution.

\subsubsection{Optimal HWI Allocation under i.i.d. Rayleigh Fad- ing}

To answer the second question, the analysis is divided into two parts. In the first part, the channels are considered as i.i.d. Rayleigh fading and majorization theory is used to evaluate the other preserving properties of the $\mathrm{SE}$ and power consumption. In the second part, a correlated Rayleigh fading model that incorporates LSF variations among the UL channels towards different BS antenna elements is assumed. Here, optimization theory is used to minimize the pilot distortion, maximize the SINR or minimize the power consumption.

This section corresponds to the first part of the analysis, the main analytical and numerical results are explained along with the main insights, and then, the answer to the second question is given under the assumption of i.i.d. Rayleigh fading channels.

In this case, the analysis considers the system model in (107) and (108), with the HWI distortion modeled as in Paper F. A lower bound on the capacity based on the analysis in Section 2.5, assuming LMMSE channel estimation and MR combining is given by

$$
\mathrm{R}_{k}=\left(1-\frac{\tau_{p}}{\tau_{u l}}\right) \log _{2}\left(1+\mathrm{SINR}_{k}\right)
$$

where

$$
\begin{aligned}
\operatorname{SINR}_{k}= & \operatorname{tr}\left(\mathbf{D}_{z}^{-1}\right)^{2} \rho_{k} \beta_{k} /\left(\operatorname{tr}\left(\mathbf{D}_{\varepsilon} \mathbf{D}_{z}^{-2}\right) \rho_{k} \beta_{k}\right. \\
& +\operatorname{tr}\left(\left(\mathbf{I}_{M}+\mathbf{D}_{\varepsilon}\right)\left(\mathbf{D}_{\varepsilon} \mathbf{D}_{z}^{-2} \frac{1}{K} \sum_{i=1}^{K} \frac{q_{i} \rho_{i} \beta_{i}^{2}}{q_{k} \beta_{k}}+\left(\sum_{i=1}^{K} \beta_{i} \rho_{i}+\sigma^{2}\right) \mathbf{D}_{z}^{-1}\right)\right),
\end{aligned}
$$


and

$\mathbf{D}_{z}=\mathbf{I}_{M}+\frac{1}{K} \mathbf{D}_{\varepsilon} \sum_{i=1}^{K} \frac{q_{i} \beta_{i}}{q_{k} \beta_{k}}+\frac{\sigma^{2}}{K q_{k} \beta_{k}}\left(\mathbf{I}_{M}+\mathbf{D}_{\varepsilon}\right)$.

Here, $\mathrm{UE}_{k}$ transmits data with an average energy per symbol of $\rho_{k}$ and pilots with an energy per symbol of $q_{k}$.

The idea is to analyze the effect of having mixed ADC bit resolution across the antenna array at the BS. For this purpose, the same relationship between HWIs and ADC bit resolution as in previous analysis is used, that is, $\varepsilon_{m}=\zeta_{m} 2^{-b_{m}}$ where $b_{m}$ is the ADC bit resolution of the $m^{\text {th }} \mathrm{RF}$ chain. Majorization theory is used to obtain insights into the effect of having mixed ADCs when a total budget of ADC bits is assumed fixed, that is, $\sum_{m=1}^{M} b_{m}=\bar{b}$ where $\bar{b}$ is the total number of ADC bits. For mathematical tractability, let us assume the ADC bit resolution to be a continuous variable such that $b_{m} \in \mathbb{R}$, then consider the following definitions.

Definition 2. Let $\bar{b} \geq M, \mathbf{b}=\left[b_{1}, \ldots, b_{M}\right]^{T}$ with $b_{1} \geq b_{2} \geq \cdots \geq b_{M} \geq 1$ and

$$
\mathcal{L}=\left\{\mathbf{b}: \sum_{m=1}^{M}[\mathbf{b}]_{m}=\bar{b}\right\} .
$$

Then, for $\mathbf{b}_{1}, \mathbf{b}_{2} \in \mathcal{L}$

$$
\mathbf{b}_{1} \succ \mathbf{b}_{2} \text { if } \sum_{m=1}^{l}\left[\mathbf{b}_{1}\right]_{m} \geq \sum_{m=1}^{l}\left[\mathbf{b}_{2}\right]_{m}, l \in\{1, \ldots, M-1\} .
$$

When $\mathbf{b}_{1} \succ \mathbf{b}_{2}$ it is said that $\mathbf{b}_{1}$ majorizes $\mathbf{b}_{2}$ [45]. In other words, the total amount of $A D C$ bits $\bar{b}$ is spread less equally in $\mathbf{b}_{1}$ than in $\mathbf{b}_{2}$.

Definition 3. A function $f(\boldsymbol{b}): \mathbb{R}^{M} \rightarrow \mathbb{R}$ is said to be Schur-convex (Schurconcave) iffor any $\mathbf{b}_{1}, \mathbf{b}_{2} \in \mathcal{L}$ it holds that

$$
\mathbf{b}_{1} \succ \mathbf{b}_{2} \Rightarrow \begin{cases}f\left(\mathbf{b}_{1}\right) \geq f\left(\mathbf{b}_{2}\right) & \text { Schur-convex } \\ f\left(\mathbf{b}_{1}\right) \leq f\left(\mathbf{b}_{2}\right) & \text { Schur-concave. }\end{cases}
$$

The idea is to find if the SE and power consumption preserve the ordering in Definition 2, that is, if it is more beneficial to have the ADC bits evenly spread across the antenna array or to concentrate them into a few high resolution ADCs. By assuming statistical channel inversion power control, that is $q_{k}=\varrho_{u l-p} / \beta_{k}$ and $\rho_{k}=\varrho_{u l-d} / \beta_{k}$, the effective SINR in (114) can be rewritten as a function of $\mathbf{b}$ given by

$$
\operatorname{SINR}_{k}(\mathbf{b})=\frac{\left(\sum_{m=1}^{M} f_{\mathrm{NUM}}\left([\mathbf{b}]_{m}\right)\right)^{2}}{\sum_{m=1}^{M} g_{\mathrm{DEN}}\left([\mathbf{b}]_{m}\right)}
$$


such that

$$
\begin{aligned}
& f_{\mathrm{NUM}}(b)=\frac{1}{(1+\overline{\mathrm{B}})\left(1+\zeta^{2} 2^{-2 b}\right)}, \\
& g_{\mathrm{DEN}}(b)=\frac{\zeta^{2} 2^{-2 b}\left(2+\zeta^{2} 2^{-2 b}\right)}{(1+\overline{\mathbf{B}})^{2}\left(1+\zeta^{2} 2^{-2 b}\right)^{2}}+\frac{\mathrm{B}}{(1+\overline{\mathbf{B}})}, \\
& \mathbf{B}=K+\frac{\sigma^{2}}{\varrho_{u l-d}}, \quad \overline{\mathbf{B}}=\frac{\sigma^{2}}{K \varrho_{u l-p}} .
\end{aligned}
$$

Notice that $f_{\mathrm{NUM}}(b)$ is a concave function and $g_{\mathrm{DEN}}(b)$ is convex for $b \geq 1.5$ (the proof is found in Paper F). Thus, it can be shown by evaluating the Schur's condition [45, Ch. 3] on (118), that the SE is a Schur-concave function which means that it is maximized when the ADC bits are spread equally across the antenna array. On the other hand, the power consumption as a function of $\mathbf{b}$ (based on the same model as in (111)) is

$$
\mathbf{P}(\mathbf{b})=\overline{\mathbf{C}}_{0}+\frac{2 K B_{\mathrm{W}}}{L} \sum_{m=1}^{M}[\mathbf{b}]_{m}+2 \mathrm{D}_{1} \sum_{m=1}^{M} 2^{\varphi_{m}[\mathbf{b}]_{m}}
$$

where $\overline{\mathrm{C}}_{0}$ includes all the power consumption terms that are independent of b. Thus, it can be shown by evaluating the Schur's condition [45, Ch. 3] that the power consumption is a Schur-convex function which is minimized when the ADC bits are spread evenly across the antenna array.

To illustrate the order preserving properties of the SE and power consumption, numerical analysis is conducted considering $K=10$ served UEs in the cell. The power parameters for pilot and data symbols are set to the same value, that is $\varrho_{u l-p}=\varrho_{u l-d}=\varrho_{u l}$. Figure 22 depicts the SE and the power consumption versus the $\mathrm{ADC}$ resolution spread parameter $\mathrm{a}_{\mathrm{SPD}} \in[0,1]$. This parameter is defined to quantify how equal the ADC bits are allocated across the antenna array. The extreme cases indicate: $i$ ) $\mathrm{a}_{\mathrm{SPD}}=0$, one antenna has $\mathrm{ADC}$ resolution $b^{\max }>1$ while all the others have one bit. ii) $\mathrm{a}_{\mathrm{SPD}}=1$, all antennas have the same $\mathrm{ADC}$ resolution. It can be seen that having equal $\mathrm{ADC}$ bit resolution across the antenna array jointly maximizes $\mathrm{SE}$ and minimizes the power consumption. However, the difference in performance is rather small (in the order of magnitude sense).

Next, to validate the insights obtained from the previous majorization analysis using the distortion model in (108) from Paper F, results from Monte Carlo simulations with the exact quantization model shown in Section 2.6 are presented. The UEs are distributed uniformly at random in a circular area with the BS at the center and the LSF coefficients are given by $\beta_{k}=\omega^{-1} d_{k}^{-\alpha}$ as in Section 2.6. The main simulation parameters are given in Table 1 located in Section 2.6. 


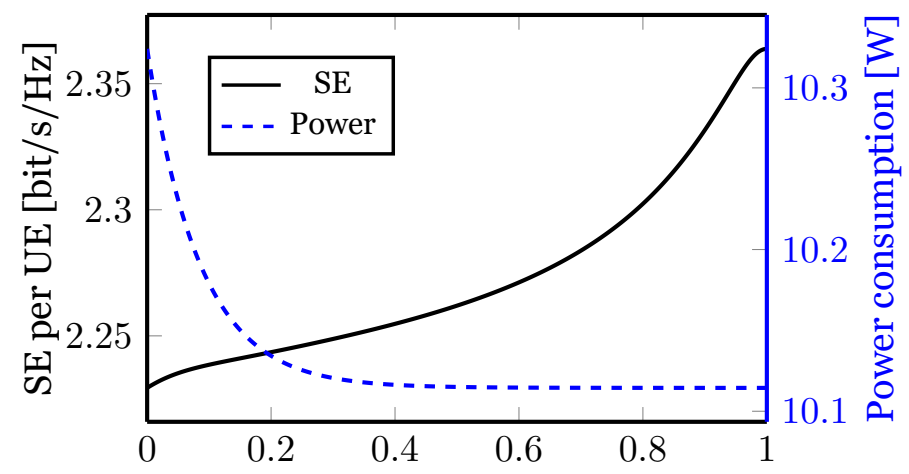

$\mathrm{ADC}$ resolution spread parameter $\mathrm{a}_{\mathrm{SPD}}$

Figure 22: $\mathrm{SE}$ and power consumption versus the ADC resolution spread parameter a $\mathrm{a}_{\mathrm{SPD}}$ for $M=100, K=10, \zeta_{m}=1.6 \forall m$, $\varrho_{u l} / \sigma^{2}=1(\mathrm{SNR}=0[\mathrm{~dB}]), b^{\max }=18$.

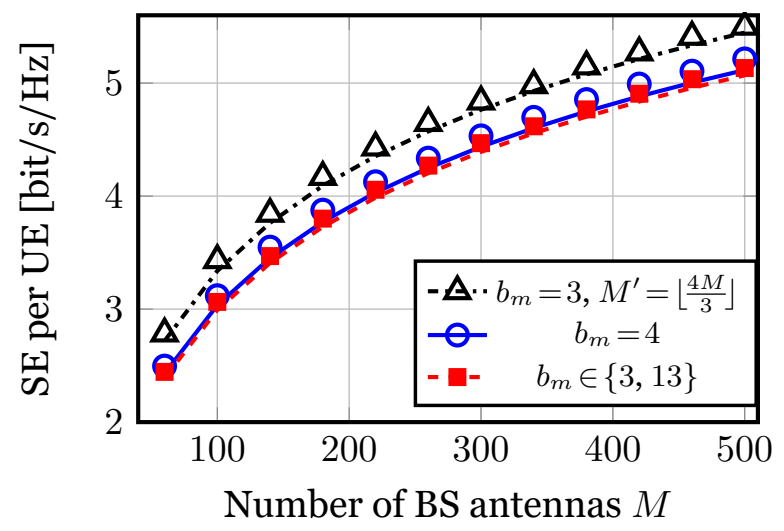

Figure 23: SE per UE versus $M$ for $\varrho_{u l} / \sigma^{2}=1(\mathrm{SNR}=0[\mathrm{~dB}])$ and $K=10$. The markers correspond to exact quantization and the lines to (114).

To compare the performance between equal and mixed ADCs, three cases are studied: $i$ ) Mixed resolution ADCs with $M$ antennas where $10 \%$ of them have 13 bits and the rest have 3 bits. ii) Equal-resolution ADCs of 4 bits with $M$ antennas. iii) Equal-resolution ADCs of 3 bits with a larger array of $M^{\prime}=\lfloor 4 M / 3\rfloor$ antennas. Note that the sum of ADC bits in the whole array is almost the same for all cases, that is $\sum_{m=1}^{M} b_{m} \approx 4 M$. In Figure 23, the SE per UE is shown versus the number of BS antennas and it can be seen that having equal $\mathrm{ADC}$ resolution gives higher $\mathrm{SE}$ even though the difference 


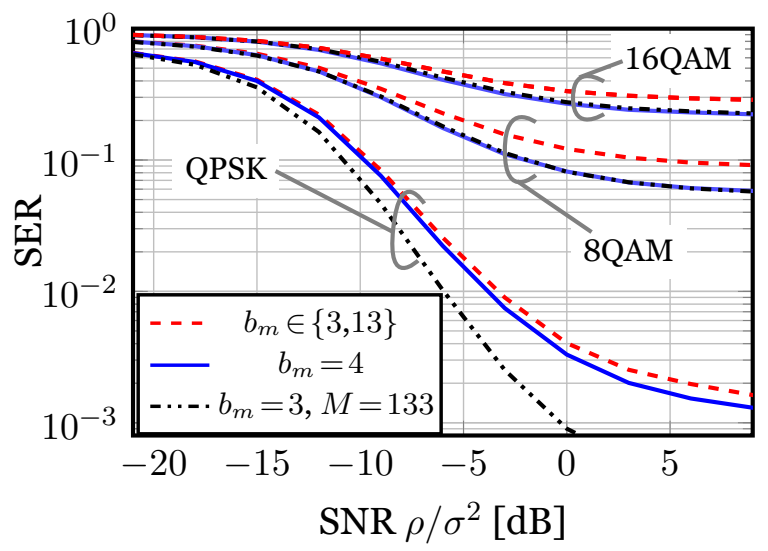

Figure 24: SER per UE versus SNR for $M=100$ and $K=10$.

tends to be small. Figure 24 shows symbol error rate (SER) curves with exact quantization and QPSK, 8-QAM, and 16-QAM modulation versus the SNR. The results show that having equal-resolution ADCs results in lower SER.

Answer 2) under i.i.d. Rayleigh fading. Given a total ADC bits budget for the BS antenna array, having equal ADC bit resolutions across the antennas maximizes SE and minimizes power consumption. Thus, the alternative design with mixed ADCs and MR combining does not offer higher SE than the baseline Massive MIMO design. However, the performance loss of having mixed ADCs is rather small (in the order of magnitude sense).

\subsubsection{Optimal HWI Allocation under Correlated Rayleigh Fading and LSF Variation among BS Antennas}

This section corresponds to the second part of the analysis to fully answer the second research question posed at the beginning of Section 3.2. Here, correlated Rayleigh fading channels with LSF variations among the UL channels towards different BS antennas are assumed. This means that, apart from including the effects of correlation between BS antennas, the diagonal elements of the correlation matrices can be different, which allows for modeling both co-located and cell-free Massive MIMO channels using the same system model. The term "cell-free" in this dissertation refers to a MU-MIMO system where the BS antennas are located in separate locations within the service area. 
To study the optimal HWI allocation, the following optimization framework is used:

$$
\begin{array}{cl}
\underset{\substack{\varepsilon=\left[\varepsilon_{1}, \ldots, \varepsilon_{M}\right]^{T} \\
\boldsymbol{\rho}=\left[\rho_{1}, \ldots, \rho_{K}\right]^{T}}}{\operatorname{maximize}} & f^{\mathrm{OBJ}}(\varepsilon, \boldsymbol{\rho}) \\
\text { subject to } & f^{\mathrm{HWI}}(\boldsymbol{\varepsilon}, \boldsymbol{\rho}) \leq 0 \\
& 0 \leq \varepsilon_{m} \leq \varepsilon_{\max } \forall m \in\{1, \ldots, M\}, \\
& 0 \leq \rho_{k} \leq P_{\max } \forall k \in\{1, \ldots, K\},
\end{array}
$$

where $P_{\max }$ is the maximal transmission energy per symbol and $\varepsilon_{\max }$ is the maximal HWI level so that $\varepsilon_{m} \leq \varepsilon_{\max } \Rightarrow b_{m} \geq 1$.

The optimization problem formulation in (123) constitutes a general framework to optimize the level of HWI per antenna and the transmission energy per data symbol. In the UL, power control is essential to avoid signals from UEs with low channel gains to be overshadowed by signals from UEs with high channel gains. In addition, power control is important to reduce the dynamic range of signals so that the ADCs introduce less quantization distortion. Thus, it is desirable to optimize the transmission energy per data symbol and the level of HWIs together. ${ }^{10}$ The function $f^{\mathrm{OBJ}}(\varepsilon, \rho)$ corresponds to the optimization objective and it can be set to maximize SE, minimize power consumption, or another useful utility function, such as minimizing the distortion or maximizing the EE. The function $f^{\mathrm{HWI}}(\varepsilon, \rho)$ enforces a constraint on the HWI level and (or) transmission energy per data symbol based on design requirements, such as, an ADC budget, or minimum SINR level, among others.

The selection of $f^{\mathrm{OBJ}}(\varepsilon, \boldsymbol{\rho})$ and $f^{\mathrm{HWI}}(\varepsilon, \boldsymbol{\rho})$ depends on the design requirements of the system. However, it is also important to choose functions that allow for favorable formulations that can be solved efficiently. In the following analysis, the optimization framework in (123) is used to obtain insights into the optimal HWI allocation to minimize the pilot distortion, maximize SINR and minimize power consumption.

\footnotetext{
${ }^{10}$ Due to mathematical tractability the transmission energy per pilot symbol is not included in the optimization framework in (123). In the case of minimizing the pilot distortion, the pilot power is excluded to obtain a closed-form solution for the HWI allocation where clear insights can be obtained. On the other hand, when the impact of the SINR is considered, the dependency between the effective SINR and $q_{k}$ is determined by a matrix inversion (see (125) and (131)) which leads to non-convex optimization problems. Thus, optimizing the transmission energy per pilot symbol is left as future work.
} 


\section{Minimizing the pilot distortion and maximizing the SINR}

Accurate CSI is crucial to harvest the benefits of Massive MIMO, thus, a first approach to optimize the HWI allocation is to consider the problem of minimizing the distortion in the channel estimation process. In this case, the transmission energy per data symbol is left fixed and it is not considered in the optimization. LMMSE channel estimation is assumed where the channel observations $\mathbf{z}_{k}$ to estimate $\mathbf{h}_{k}$ are found by projecting $\tau_{p}$ instances of (107) corresponding to the received pilot signal with $\phi_{k}^{*} /\left(\tau_{p} \sqrt{q_{k}}\right)$ (See Paper G for details). Thus, the LMMSE channel estimate of $\mathbf{h}_{k}$ is

$$
\hat{\mathbf{h}}_{k}=\mathbb{E}\left\{\mathbf{h}_{k} \mathbf{z}_{k}^{H}\right\}\left(\mathbb{E}\left\{\mathbf{z}_{k} \mathbf{z}_{k}^{H}\right\}\right)^{-1}=\mathbf{R}_{k} \Psi_{k}^{-1} \mathbf{z}_{k}
$$

with

$$
\mathbf{\Psi}_{k}=\mathbf{R}_{k}+\underbrace{\frac{1}{\tau_{p} q_{k}}\left(\sigma^{2} \mathbf{I}_{M}+\sum_{i=1}^{K} q_{i} \mathbf{D}_{\mathbf{R}_{i}} \mathbf{D}_{\varepsilon}\right)}_{\text {Noise and pilot distortion }}
$$

where $\mathbf{D}_{\mathbf{R}_{i}}=\operatorname{diag}\left(\left[\mathbf{R}_{i}\right]_{11}, \ldots,\left[\mathbf{R}_{i}\right]_{M M}\right)$.

Due to the HWIs effect in the received pilot signal, the channel estimate $\hat{\mathbf{h}}_{k}$ is corrupted by a distortion that depends on the channels from all UEs in the cell. To minimize the MSE of the channel estimates, the noise and hardware distortion effect should be as low as possible (corresponding to the last two terms in (125)). The term "pilot distortion" is used to specify the effect of hardware distortion on the channel estimation.

To minimize the pilot distortion, the objective and constraint functions in (123) are set as

$$
\begin{aligned}
f^{\mathrm{OBJ}}(\boldsymbol{\varepsilon}) & =\operatorname{tr}\left(\sum_{i=1}^{K} q_{i} \mathbf{D}_{\mathbf{R}_{i}} \mathbf{D}_{\varepsilon}\right), \\
f^{\mathrm{HWI}}(\varepsilon) & =\sum_{m=1}^{M} \log _{2}\left(\frac{\zeta_{m}}{\varepsilon_{m}}\right)-b_{\text {тот }} .
\end{aligned}
$$

The objective minimizes the pilot distortion (see the third term in (125)) while the constraint imposes a lower limit on the combined level of HWIs for all antennas through an ADC bit budget $\sum_{m=1}^{M} b_{m} \leq b_{\text {тот }} \in \mathbb{N}$. In addition, for a more tractable analysis, the ADC bits $b_{m}$ have been relaxed to be realvalued and the maximal HWI level constraint $\varepsilon_{m} \leq \varepsilon_{\max }$ is removed, that is $\varepsilon_{\max }=\infty$. The resulting optimization problem is convex and it has a closed-form solution that is given in the next theorem (the proof of convexity is shown in Paper G). 
Theorem 3. The optimal solution of the convex optimization problem in (123) with objective in (126), constraint in (127) and $\varepsilon_{\max }=\infty$, is given by

$$
\begin{aligned}
& \varepsilon_{m}^{\mathrm{OP}}=\left(2^{-b_{\text {тот }}} \prod_{m^{\prime}=1}^{M} \zeta_{m^{\prime}} \sqrt{\frac{p_{m^{\prime}}^{\mathrm{U}}}{p_{m}^{\mathrm{U}}}}\right)^{\frac{1}{M}} \\
& b_{m}^{\mathrm{OP}}=\frac{1}{M}\left(b_{\text {тот }}+\sum_{\substack{m^{\prime}=1 \\
m^{\prime} \neq m}}^{M} \log _{2}\left(\frac{\zeta_{m}}{\zeta_{m^{\prime}}} \sqrt{\frac{p_{m}^{\mathrm{U}}}{p_{m^{\prime}}^{\mathrm{U}}}}\right)\right)
\end{aligned}
$$

where $p_{m}^{\mathrm{U}}=\sum_{i=1}^{K} q_{i}\left[\mathbf{R}_{i}\right]_{m m}$ is the average undistorted received pilot power.

Proof. It is provided in Paper G.

The optimal solution $\varepsilon_{m}^{\mathrm{OP}}$ satisfies the total HWI budget constraint with equality (see Appendix A in Paper G) which means that the full ADC bit budget $b_{\text {тот }}$ is used. The optimal level of HWIs is allocated based on the received signal strength per antenna, so that higher received power at a given antenna results in lower level of HWI (more ADC bits) allocation and vice-versa. The proportion of HWI allocation is based on the product of the received power among antennas due to the exponential relationship between the level of HWI $\varepsilon_{m}$ and the ADC bit $b_{m}$ (i.e., $\varepsilon_{m}=\zeta_{m} 2^{-b_{m}}$ ).

Remark 5. If the average undistorted received pilot power (i.e., $p_{m}^{\mathrm{U}}$ ) has low variations across the BS antennas, then equal ADC bit allocation is close to optimal for minimal pilot distortion. In the special case of equal received power, equal ADC bit allocation is optimal.

To evaluate the HWI allocation in terms of SE, an achievable rate analysis as in Section 2.5 is done. Two different options are considered for selecting the combining vector as

$$
\mathbf{v}_{k}= \begin{cases}\hat{\mathbf{h}}_{k} & \mathrm{MR} \\ \left(\sum_{i=1}^{K} \hat{\mathbf{h}}_{i} \hat{\mathbf{h}}_{i}^{H} \rho_{i}+\sigma^{2} \mathbf{I}_{M}\right)^{-1} \hat{\mathbf{h}}_{k} \rho_{k} & \mathrm{RZF} .\end{cases}
$$

The regularized zero-forcing (RZF) method aims at suppressing inter-user interference while maintaining a decent SNR level for the desired signal. The following theorem introduces an achievable SE under HWIs that is valid for any linear combining method. 
Theorem 4. An achievable $S E$ for $\mathrm{UE}_{k} \forall k \in\{1, \ldots, K\}$ under the hardware distortion model in (108) from Paper $G$ is

$$
\begin{aligned}
\mathrm{SE}_{k} & =\left(1-\frac{\tau_{p}}{\tau_{c}}\right) \log _{2}\left(\mathrm{SINR}_{k}\right) \\
\mathrm{SINR}_{k} & =\frac{\left|\mathbb{E}\left\{\mathbf{v}_{k}^{H} \mathbf{h}_{k}\right\}\right|^{2} \rho_{k}}{\sum_{i=1}^{K} \mathbb{E}\left\{\left|\mathbf{v}_{k}^{H} \mathbf{h}_{i}\right|^{2}\right\} \rho_{i}-\left|\mathbb{E}\left\{\mathbf{v}_{k}^{H} \mathbf{h}_{k}\right\}\right|^{2} \rho_{k}+\mathbb{E}\left\{\left|\mathbf{v}_{k}^{H} \mathbf{n}\right|^{2}\right\}+\mathbb{E}\left\{\left|\mathbf{v}_{k}^{H} \mathbf{e}\right|^{2}\right\}} .
\end{aligned}
$$

Proof. The proof is given in Paper G.

In particular, for the case of MR combining, the following corollary provides a closed-form expression for the effective SINR.

Corollary 1. The effective SINR in (130) with MR combining is given by

$$
\begin{aligned}
& \overline{\operatorname{SINR}}_{k}=\rho_{k} \operatorname{tr}\left(\mathbf{R}_{k} \mathbf{\Psi}_{k}^{-1} \mathbf{R}_{k}\right)^{2} /(\underbrace{\rho_{k} \operatorname{tr}\left(\mathbf{D}_{\varepsilon} \operatorname{abs}\left(\operatorname{diag}\left(\mathbf{R}_{k} \mathbf{\Psi}_{k}^{-1} \mathbf{R}_{k}\right)\right)^{2}\right)}_{\text {Self-distortion }\left(\mathrm{UE}_{k}\right)} \\
& +\sum_{i=1}^{K} \frac{\rho_{i} q_{i}}{\tau_{p} q_{k}} \operatorname{tr}(\underbrace{\mathbf{D}_{\varepsilon} \operatorname{abs}\left(\operatorname{diag}\left(\mathbf{R}_{i} \mathbf{R}_{k} \mathbf{\Psi}_{k}^{-1}\right)\right)^{2}}_{\text {Distortion from inter-user interference }}) \\
& +\sum_{i=1}^{K} \frac{\rho_{i} q_{i}}{\tau_{p} q_{k}} \operatorname{tr}(\underbrace{\mathbf{D}_{\varepsilon} \operatorname{diag}\left(\operatorname{abs}\left(\mathbf{R}_{k} \mathbf{\Psi}_{k}^{-1}\right)^{2} \mathbf{D}_{\varepsilon} \operatorname{abs}\left(\mathbf{R}_{i}\right)^{2}\right)}_{\text {Additional distortion from all UEs }}) \\
& \left.\left.+\operatorname{tr}(\underbrace{\sum_{i=1}^{K} \rho_{i} \mathbf{R}_{i}}_{\text {Inter-user interference }}+\underbrace{\sigma^{2} \mathbf{I}_{M}}_{\text {Noise }}+\underbrace{\sum_{i=1}^{K} \rho_{i} \mathbf{D}_{\varepsilon} \mathbf{D}_{\mathbf{R}_{i}}}_{\text {Data distortion }}) \mathbf{R}_{k} \mathbf{\Psi}_{k}^{-1} \mathbf{R}_{k}\right)\right)
\end{aligned}
$$

where the operator abs $(\cdot)^{2}$ corresponds to element wise squared absolute value.

Proof. The proof is provided in Paper G.

The effect of HWIs in the SE is more involved than in the channel estimates as it is shown in the SINR expression in (131). Since the hardware distortion affects both the channel estimates and data signals, cross products arise in the linear combining process which, in turn, form the additional 
distortion terms shown in (131). In what follows, optimization problems to maximize the effective SINR in (131) are introduced.

As shown in Theorem 4, the SE has a logarithmic dependency on the effective SINR. Since the logarithm is a concave and monotonically increasing function, it is often advantageous to optimize the effective SINRs directly to find more tractable formulations. To define the objective function $f^{\mathrm{OBJ}}(\varepsilon, \boldsymbol{\rho})$, it is necessary to find a good compromise between fairness and aggregated performance of the UEs. Thus, the same objectives as in Section 3.1.3 are used such that

$$
f^{\mathrm{OBJ}}(\varepsilon, \boldsymbol{\rho})=\left\{\begin{array}{cll}
\prod_{k=1}^{K} & \overline{\operatorname{SINR}}_{k}(\varepsilon, \boldsymbol{\rho}) & \text { Max-prod SINRs } \\
\min _{k \in\{1, \ldots, K\}} & \overline{\operatorname{SINR}}_{k}(\varepsilon, \boldsymbol{\rho}) & \text { Max-min fairness. }
\end{array}\right.
$$

The first objective in (132) is the max-product of SINRs (i.e., the geometric mean) and it aims at maximizing the aggregated performance of all UEs while maintaining a certain level of fairness since UEs with very low SINR would make the objective small. The second objective in (132) is max-min fairness and it seeks to maximize the lowest SINR among all UEs. The constraint function $f^{\mathrm{HWI}}(\varepsilon)$ is set as in (127) to satisfy an ADC bit budget.

The effective SINR in (131) depends on $\boldsymbol{\Psi}_{k}$, which in turn, is a function of the HWI level through the pilot distortion term in (125). As a result, the problems formulated as in (123) with the objectives given in (132) and constraint in (127), are not convex. The impact of HWI in $\boldsymbol{\Psi}_{k}$ is encompassed by the pilot distortion which has been already analyzed. Thus, to gain more insights into the HWI level allocation for maximal SINR, the optimization problems can be solved assuming that $\boldsymbol{\Psi}_{k}$ is fixed. In this case, the problem for both objectives in (132) and constraint in (127) can be casted as geometric programs (See Definition 1). This follows from the fact that the denominator of the effective SINR is a posynomial function of $\rho_{k}$ and $\varepsilon_{m}$. The details on how to formulate these geometric programs can be found in Paper G.

To illustrate the impact of spatial channel correlation and LSF variations, the model $\mathbf{R}_{k}=\mathbf{D}_{\beta_{k}}^{\frac{1}{2}} \overline{\mathbf{R}}_{k} \mathbf{D}_{\beta_{k}}^{\frac{1}{2}}$ is taken as an example for the spatial channel correlation matrices which is based on [14]. The LSF coefficients between $\mathrm{UE}_{k}$ and the $m^{\text {th }}$ BS antenna element is denoted by $\beta_{m k}$ such that $\mathbf{D}_{\beta_{k}}=\operatorname{diag}\left(\beta_{1 k}, \ldots, \beta_{M k}\right)$. The correlation matrix $\overline{\mathbf{R}}_{k}$ has $\left[\overline{\mathbf{R}}_{k}\right]_{m m}=1$ and $\left|\left[\overline{\mathbf{R}}_{k}\right]_{m m^{\prime}}\right| \leq 1 \forall m \neq m^{\prime} \in\{1, \ldots, M\}$ to account for the correlation between antenna elements. The average channel gain is given by $\bar{\beta}_{k}=\operatorname{tr}\left(\mathbf{R}_{k}\right) / M$. Two cases are considered for the spatial correlation matrices: ${ }^{11}$

\footnotetext{
${ }^{11}$ Two more cases are considered in Paper G.
} 
Table 3: Optimal HWI allocation simulation parameters.

\begin{tabular}{c|c} 
Parameter & Value \\
\hline System bandwidth & $B_{\mathrm{W}}=20[\mathrm{MHz}]$ \\
Max. trans. power per UE & $10 \log _{10}\left(P_{\max } B_{\mathrm{W}}\right)=20[\mathrm{dBm}]$ \\
Noise power & $10 \log _{10}\left(\sigma^{2} B_{\mathrm{W}}\right)=-94[\mathrm{dBm}]$ \\
Square side length & $0.4[\mathrm{~km}]$ \\
Pathloss exponent & $\alpha=3.76$ \\
Pathloss at $1 \mathrm{~km}$ & $\omega=148.1[\mathrm{~dB}]$ \\
Shadow fading std. dev. & $\sigma_{\mathrm{sh}}=10[\mathrm{~dB}]$ \\
Angular std. deviation & $\sigma_{\mathrm{ang}}=10^{\circ}$ \\
ADC constant & $\zeta_{m}=1.6 \forall m \in\{1, \ldots, M\}$ \\
\hline
\end{tabular}

\section{Co-located antennas and spatial correlation (Co-corr-I)}

The matrix $\overline{\mathbf{R}}_{k}$ follows the Gaussian local scattering model defined in Section 2.3.2, with angular standard deviation $\sigma_{\text {ang. The LSF coeffi- }}$ cients are equal for all antenna elements such that $\mathbf{D}_{\beta_{k}}=\bar{\beta}_{k} \mathbf{I}_{M}$. The average channel gain is modeled as $\bar{\beta}_{k}=\omega^{-1} d_{k}^{-\alpha} \digamma^{\text {sh }}$ where $\omega$ is the fixed pathloss at $1 \mathrm{~km}, \alpha$ is the pathloss exponent, and $d_{k}$ is the distance between $\mathrm{UE}_{k}$ and the BS (measured in [km]). The shadow fading effects are included in $\digamma^{\text {sh }}$ and modeled as independent log-normal distributed with standard deviation $\sigma_{\text {sh }}[\mathrm{dB}]$, that is, $10 \log _{10}\left(\digamma^{\mathrm{sh}}\right) \sim \mathcal{N}\left(0, \sigma_{\mathrm{sh}}^{2}\right)$. Note that since the antennas are co-located, the distance $d_{k}$ is considered to be the same for all antennas at the BS.

\section{Cell-free and uncorrelated channels (Cell-free)}

The matrix $\overline{\mathbf{R}}_{k}=\mathbf{I}_{M}$ and the antenna elements are assumed to be located at different positions so that $\beta_{m k}=\omega^{-1} d_{m k}^{-\alpha} \digamma^{\text {sh }}$ where $d_{m k}$ corresponds to the distance between $\mathrm{UE}_{k}$ and the $m^{t h}$ antenna element. The shadow fading effects are included in $\digamma^{\text {sh }}$ and are modeled as in the case Co-corr-I.

To gain insights into how the optimal HWI allocation to minimize the pilot distortion or maximize the SINR affect the SE, Monte-Carlo simulations are performed. The UEs are dropped uniformly at random in a squared area. In the case of co-located antennas, the BS is located at the center of the square whereas in the cell-free case the antennas are distributed uniformly at random. The transmission energy per pilot symbol is set by the statistical channel inversion power control such that $q_{k}=\min \left\{P_{\max }, \bar{q} / \bar{\beta}_{k}\right\}$ where $\bar{q}$ is used to set the desired SNR level and $P_{\max }$ is the maximum transmission 


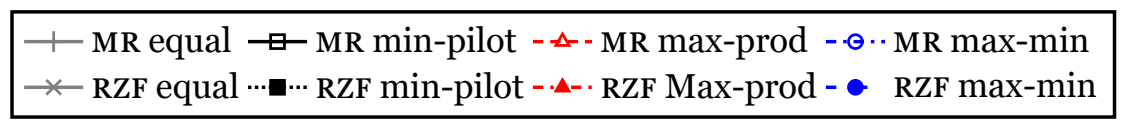

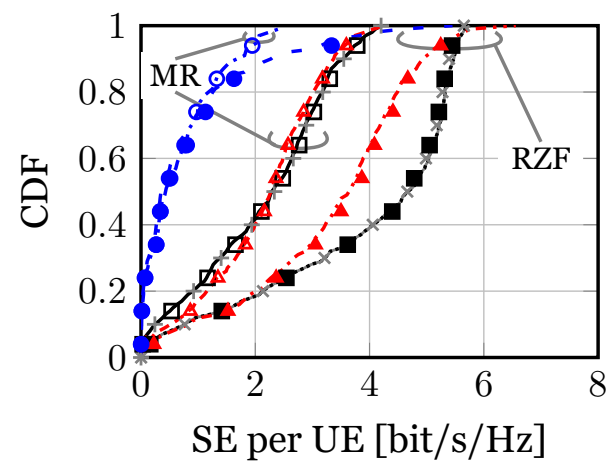

(a) Channel model Co-corr-I.

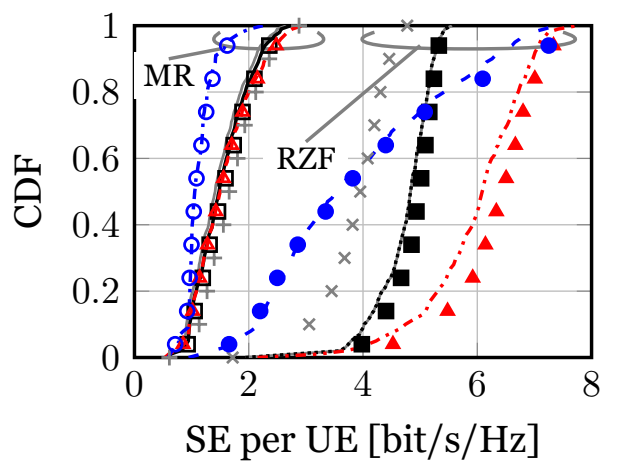

(b) Channel model Cell-free.

Figure 25: CDF of the SE per UE for $M=100, K=10, \tau_{c}=200$, $\bar{q} / \sigma^{2}=1(\mathrm{SNR}=0[\mathrm{~dB}])$, and $b_{\mathrm{TOT}}=3 M$. The lines correspond to the results in Theorem 4 and Corollary 1 (for MR) with the distortion model in (108) from Paper G. The markers are the results with exact quantization and integer bit allocation (see Section 3.2 in Paper G)

energy per symbol. In the SE calculations corresponding the minimum pilot distortion, the average transmission energy per data symbol is set equal to the one for pilots, such that $\rho_{k}=q_{k}$. Table 3 summarizes the main simulation parameters used.

To validate the accuracy of the results obtained with the HWI model in (108) from Paper G, the SE with exact quantization is also calculated following the analysis in Sections 2.5 and 2.6 (see Paper G for details). In all the results with exact quantization, the ADC bit resolution is set to integer values. Thus, a heuristic algorithm is proposed in Paper G to map a realvalued $\mathrm{ADC}$ bit allocation into integer values that satisfy the ADC bit budget (see Section 3.2 in Paper G for details).

Figure 25 shows the empirical CDF of SE per UE for the distortion model in (108) from Paper $G$ with the expressions found in Theorem 4 for RZF and Corollary 1 for MR (depicted by lines). These results are compared to the exact quantization model introduced in Section 2.6 (depicted by the markers).

It can be seen that the SE with the distortion model in (108) from Paper G 
is accurate in most cases. However, in the Cell-free case with equal ADC bit allocation, there is a gap between the exact quantization and the distortion model in (108). A possible explanation is that the distortion becomes practically independent when it is combined from many different signals that have similar strength, which might not be the case in Cell-free since the UEs are located at different distances to the antennas. This discrepancy is substantially reduced when the ADC bit allocation is optimized since the antennas with higher received power are allocated more ADC bits that allow them to increase their dynamic range.

By comparing the HWI allocation methods, it can be seen that, maxmin fairness reduces the SE of UEs with high channel gains (shown in the high percentile regions) in order to increase the performance of the UE with minimum SE. However, except for very low SE percentile values, maxproduct of SINR and minimum pilot distortion are able to offer higher SE.

Notice that the optimization objectives in (132) are based on a SINR expression for MR which do not optimize the system with RZF. Nevertheless, it is important to assess the potential benefits of interference suppression methods such as RZF.

In the case of MR, max-product of SINR and minimum pilot distortion offer almost the same SE for all UEs. The max-product of SINRs with MR tends to assign more power to UEs with higher channel gains which may not be the best approach to suppress interference. On the other hand, in the case with co-located BS antennas the level of interference from a given UE varies less that in the Cell-free case where some BS antennas might be located much further than others. As a result, RZF with minimum pilot distortion provides higher SE for most UEs under Co-corr-I, whereas the max-product of SINRs provides the highest SE under the Cell-free case.

In summary, statistical channel inversion power control combined with minimum pilot distortion HWI allocation offers the same or higher SE as max-product of SINRs when MR combining is used. Improvements of up to 1 [bpcu] per UE for the Cell-free case using minimum pilot distortion ADC bit allocation and RZF compared to equal ADC bit allocation are shown in Figure 25b. Furthermore, optimizing the max-product of SINRs with RZF increases the SE for most UEs more than 1 [bpcu] compared to minimum pilot distortion, and more than 2 [bpcu] compared to equal ADC bit allocation.

\section{Impact of power consumption}

To assess the impact of power consumption into the optimal ADC bit allocation, two optimization problems are formulated based on the framework given in (123). The first problem is to minimize the power consumption 
subject to a product of SINR constraint, and the second is to maximize the product of SINRs subject to a power consumption constraint. Note that although these problems seem very similar, they have different meaning and by comparing the performance between them, we can identify the key conditions in which optimizing the $\mathrm{ADC}$ bit resolution across $\mathrm{BS}$ antennas would give the most benefits. For these optimization problems the ADC bit budget is optimized along with the ADC bit allocation which inherently represents a mixed-ADC approach. Thus, to provide a benchmark for comparison, the solution to both optimization problems considering equal ADC bits across antennas is also included.

The power consumption is modeled as in Section 2.6, such that, the power consumption that depends on the HWI level and transmission energy per data symbol is given by

$$
\mathrm{P}_{\mathrm{TXD}-\mathrm{ADC}}(\varepsilon, \boldsymbol{\rho})=\underbrace{\left(1-\frac{\tau_{p}}{\tau_{c}}\right) \frac{B_{\mathrm{W}}}{\eta} \sum_{k^{\prime}=1}^{K} \rho_{k}}_{\text {Transmission data power }}+\underbrace{2 \mathrm{D}_{1} \sum_{m=1}^{M} \zeta_{m} \varepsilon_{m}^{-1}}_{\text {Total ADC power }}
$$

where $\eta$ is the efficiency of the power amplifier, which is set equal for all BS antennas. The power function $\mathrm{P}_{\mathrm{TXD}-\mathrm{ADC}}(\varepsilon, \rho)$ is linear in $\rho_{k}$ and monotonically decreasing in $\varepsilon_{m}$. Thus, lower transmission power and higher HWI level results in lower power consumption. Moreover, from Definition 1, it can be seen that $\mathrm{P}_{\text {TXD-ADC }}(\varepsilon, \boldsymbol{\rho})$ is also a posynomial function of $\rho_{k}$ and $\varepsilon_{m}$.

For the first optimization problem, the objective function is given by

$$
f^{\mathrm{OBJ}}(\varepsilon, \rho)=-\mathrm{P}_{\mathrm{TXD}-\mathrm{ADC}}(\varepsilon, \boldsymbol{\rho})
$$

and the constraint function is

$$
f^{\mathrm{HWI}}(\varepsilon, \boldsymbol{\rho})=1-\left(\prod_{k=1}^{K}\left(\frac{\overline{\operatorname{SINR}}_{k}(\varepsilon, \boldsymbol{\rho})}{\gamma_{\mathrm{SINR}}}\right)\right)
$$

which enforces the product of SINRs to be above $\gamma_{\mathrm{SINR}}^{K}$. The power consumption minimization problem can be casted as geometric program by noticing that the denominator of the effective SINR is a posynomial function of $\rho_{k}$ and $\varepsilon_{m}$ (see details in Paper G), and so is the objective function.

For better interpretation, the SINR target $\gamma_{\mathrm{SINR}}$ in (135) can be mapped into a target SE as

$$
\gamma_{\mathrm{SE}}=\left(1-\frac{\tau_{p}}{\tau_{c}}\right) \log _{2}\left(1+\gamma_{\mathrm{SINR}}\right)
$$


Notice that since the logarithm is a monotonically increasing function, by rising $\gamma_{\mathrm{SINR}}$, the SE grows. At the same time, when $\gamma_{\mathrm{SE}}$ increases for a fixed number of BS antennas, the minimum power consumption is also increased since higher SE demands more power and lower HWI level. However, recall from the general optimization framework in (123), that there are maximum limits for the HWI levels per antenna and transmission energy per symbol. Thus, to afford higher $\gamma_{\mathrm{SE}}$ values beyond a certain point while keeping the optimization problem feasible, the operating point of the system needs to be adapted. This can be accomplished by increasing the number of BS antennas or reducing the number of spatially multiplexed UEs.

For the second optimization problem the max-product of SINRs objective function is defined as in (132), and the constraint function is given by

$$
f^{\mathrm{HWI}}(\varepsilon, \boldsymbol{\rho})=\mathrm{P}_{\mathrm{TXD}-\mathrm{ADC}}(\varepsilon, \boldsymbol{\rho})-\gamma_{\mathrm{PC}}
$$

where $\gamma_{\mathbf{P C}}$ is the maximum power consumption that can be spent on ADCs plus transmission data power. Similar to the minimum power consumption problem, the max-product of SINRs subject to a power consumption constraint can also be casted as a geometric program (see Paper $\mathrm{G}$ for details).

To obtain insights into the balance between the total power consumption and the sum SE, the EE is used as a performance metric. The EE is defined as

$$
\mathrm{EE}=\frac{B_{\mathrm{W}} \sum_{k=1}^{K} \mathrm{SE}_{k}}{\mathrm{P}_{\mathrm{CST}}+\mathrm{P}_{\mathrm{UE}} K+\mathrm{D}_{0} M+\mathrm{P}_{\mathrm{PILOT}}+\mathrm{P}_{\mathrm{TXD}-\mathrm{ADC}}(\varepsilon, \boldsymbol{\rho})+\mathrm{P}_{\mathrm{CD}} B_{\mathrm{W}} \sum_{k=1}^{K} \mathrm{SE}_{k}}
$$

where the power consumption model follows the same formulations as in (33) in Section 2.6. $\mathrm{P}_{\mathrm{PILOT}}=\frac{\tau_{p}}{\tau_{c}} \frac{B_{\mathrm{W}}}{\eta} \sum_{k^{\prime}=1}^{K} q_{k}$ is the transmission power of pilot symbols. The term $\mathrm{P}_{\mathrm{CST}}$ accounts for the circuit power consumption that does not depend on the ADC bit resolution or transmission power, such as site cooling and baseband processing (see Section 2.6). The values of the main parameters used in the numerical analysis are included in Table 4 and are selected based on [8, Ch 5], [84].

To analyze the impact of power consumption in the optimal HWI allocation, Monte-Carlo simulations are performed with the same setup as in the previous section that evaluates the SE. All results in Figures 26 and 27 correspond to the exact quantization model and integer bit allocation (see Section 3.2 in Paper G).

Figure 26 shows the EE versus number of BS antennas and UEs with MR combining. The EE is obtained from the solution to the power minimization problem previously formulated in this section, with objective and constraint functions given in (134) and (135), respectively. It can be seen, that for 
Table 4: Power consumption model parameters.

\begin{tabular}{c|c} 
Parameter & Value \\
\hline Power amplifier efficiency & $\eta=0.39$ \\
ADC power constant & $\mathrm{D}_{1}=0.006[\mathrm{~W} /$ conv-step $]$ \\
Fixed circuit power & $\mathrm{P}_{\mathrm{CST}} \in[5,20][\mathrm{W}]$ \\
coding/decoding power & $\mathrm{P}_{\mathrm{CD}}=1.15[\mathrm{Joule} / \mathrm{Gbit}]$ \\
Power per UE & $\mathrm{P}_{\mathrm{UE}}=0.1[\mathrm{~W}]$ \\
Power per BS antenna & $\mathrm{D}_{0}=0.05[\mathrm{~W}]$ \\
\hline
\end{tabular}

a fixed target SE $\gamma_{\mathrm{SE}}=1$ [bit/s/Hz], increasing $M$ decreases EE whereas increasing $K$ shows a unimodal (i.e., increases, saturates, and decreases) EE behavior. This means that the main EE benefit of adding more BS antennas is to allow further spatial multiplexing. It is worth mentioning that in general the EE has a unimodal dependency with both $M$ and $K$ [83]. However, since in this case the EE is shown for minimum power consumption subject to a product of SINR constraint, the resulting EE does not fully show this unimodal behavior within the feasibility region of the optimization problem.

By comparing the EE between equal and mixed-ADC bit allocation in Figure 26, it can be seen that for co-located scenarios the EE is almost the same whereas in the Cell-free case having equal $\mathrm{ADC}$ bits gives higher $\mathrm{EE}$. However, it is interesting to see that in the Cell-free case the EE with equal ADC bit resolution saturates at $K=20$ whereas the EE with mixed-ADCs continues to increase beyond $K=25$. Thus, the most EE benefit of minimizing the power consumption in the Cell-free case is achieved for high number of UEs.

In Figure 27, the EE versus the target power consumption is shown with RZF. The EE corresponds to the solution of the max-product of SINRs problem with objective function given in (132) and constraint in (137). Note that since the power consumption and SE both increase when the ADC bit resolution increases, the optimal solution would satisfy the power constraint with equality. Thus, the optimal SE follows the same behavior as the EE depicted in Figure 27. The EE has unimodal behavior which means that the target power consumption can be tunned to maximize SE and EE. Comparing equal and mixed-ADC bit allocations, it can be seen that for co-located scenarios the EE is almost the same whereas in the Cell-free case, mixed-ADCs increase the EE up to 30\% (at its maximum) compared to equal ADC bit allocation.

It is interesting to see that minimizing the power consumption under a product of SINRs constraint and maximizing the product of SINRs under a power consumption constraint, give such different results when comparing 


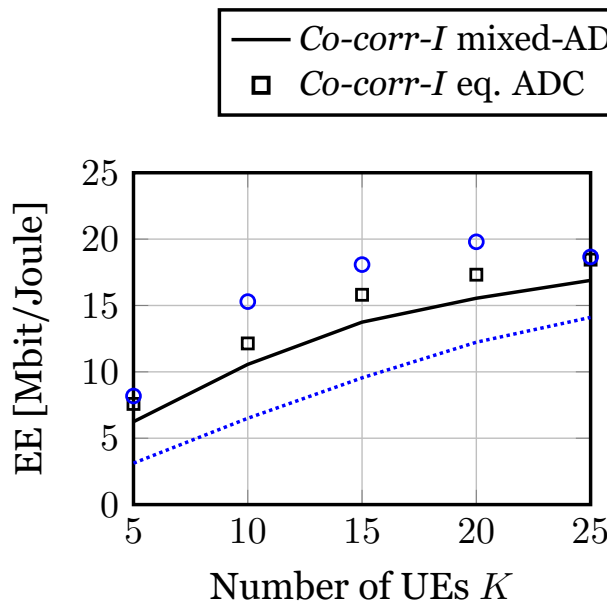

(a) Energy Efficiency vs $K$ with $M=100$.

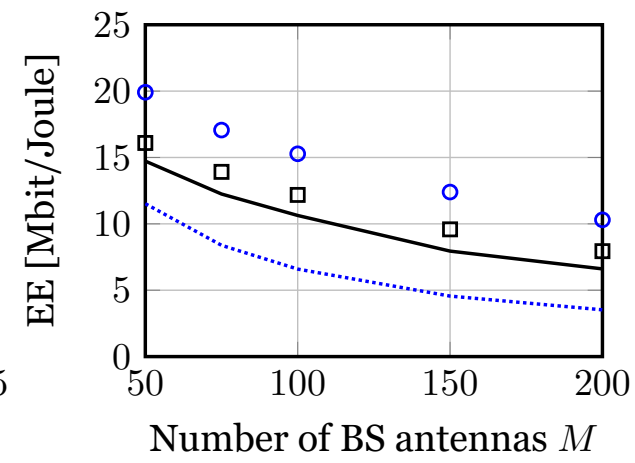

(b) Energy Efficiency vs $M$ with $K=10$.

Figure 26: $\mathrm{EE}$ vs $M$ and $K$ with MR combining, $\gamma_{\mathrm{SE}}=1[\mathrm{bit} / \mathrm{s} / \mathrm{Hz}], \tau_{c}=200, \bar{q}=\sigma^{2}(\mathrm{SNR}=0[\mathrm{~dB}])$ and $\mathrm{D}_{1}=0.006[\mathrm{~W} /$ conv-step]. All plots consider exact quantization and integer bit allocation (see Section 3.2 in Paper G).

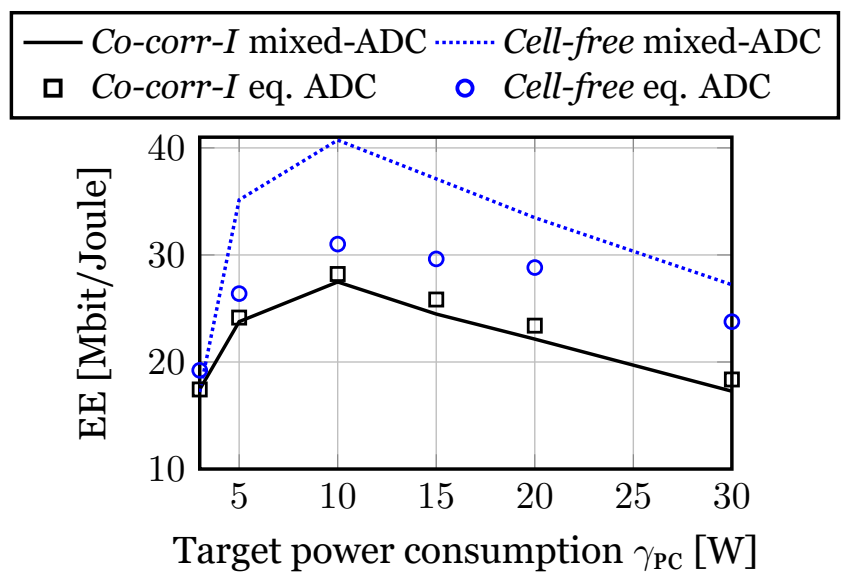

Figure 27: EE vs $\gamma_{\mathrm{PC}}$ with RZF, $M=100, K=10, \tau_{c}=200$ and $\bar{q}=\sigma^{2}(\mathrm{SNR}=0[\mathrm{~dB}])$. All plots consider the exact quantization model and integer bit allocation (see Section 3.2 in Paper G).

mixed-ADC and equal-ADC architectures. This indicates that mixed-ADCs are mostly useful to increase the SE in propagation environments with large 
variations between channels towards BS antennas, while equal-ADCs are better suited for power savings. In both cases high EE can be achieved either by selecting the number of BS antennas and UEs, or the target power consumption.

Answer 2) under correlated Rayleigh fading. In cases with low LSF variations among the UL channels towards different BS antennas, equal $\mathrm{ADC}$ bit allocation is close to optimal. However, when the are large LSF channel variation among BS antennas optimizing the HWI allocation combined with interference suppression techniques can provide high SE gains. In particular, for cell-free Massive MIMO scenarios with RZF, mixed-ADCs can achieve a 2 [bit/s/HZ] SE gain compared to equal ADC bit allocation.

In terms of EE, when minimizing the power consumption under a product of SINRs constraint, equal ADC bit allocation provides higher EE compared to mixed-ADCs in the case of cell-free Massive MIMO. On the other hand, when maximizing the product of SINRs under a power consumption constraint, the EE with mixed-ADCs using RZF is increased up to $30 \%$ (at its maximum point) compared to equal ADC bit allocation. 


\section{Chapter 4}

\section{Summary, Conclusions, and Future Work}

The high demand for data traffic and connected devices calls for the development of new ICT technologies. Massive MIMO is a key component of $5 \mathrm{G}$ wireless networks due to its ability to jointly increase SE and EE. In this dissertation, two alternative designs for Massive MIMO systems are studied and compared to the baseline Massive MIMO operation in terms of SE, power consumption, and EE.

The first alternative design is SP, which is based on superimposing pilot and data symbols in the UL to remove the conventional pilot overhead and reduce the pilot contamination. To evaluate the performance with SP, novel closed-form expressions for lower bounds on the UL capacity using MR combining are found. The results show that SP is able to reduce the pilot contamination at the expense of adding extra coherent and non-coherent interference that limits the SE gain over the baseline Massive MIMO operation with RP. A comparative analysis between RP and SP in terms of the UL SE and EE indicates that, when both system are optimized, RP and SP offer comparable performance and in most cases RP with optimal pilot length is the preferred choice. Furthermore, a multiobjective optimization framework is used to compare the joint UL and DL SE between RP and SP. The results show that in most cases of interest, when the number of spatial multiplexed UEs is smaller than the coherence block size, conventional RP attains higher UL and DL SE. However, the case in which the coherence block size and number of spatial multiplexed UEs are approximately the same, then SP offers higher UL and DL SE. Finally, the MICED algorithm is proposed to harvest the SE benefits of data-aided channel estimation with both RP and SP. The results show that both SP and RP combined with the proposed MICED algorithm 
offer higher SE compared to the Massive MIMO baseline. In addition, the highest SE gain is found with SP in cases with short coherence block size which correspond to high mobility or low carrier frequency. Moreover, the MICED algorithm with SP can support aggressive spatial multiplexing which in turn enables other services like machine type communications.

The second alternative design refers to the use of mixed-ADCs at the $\mathrm{BS}$ antennas. The idea is to allow each RF chain to have a different ADC bit resolution and find the optimal allocation of ADC bits across the BS antennas. In this case, first a general study of the optimal tradeoff between the level of HWIs and number of BS antennas considering the SE and power consumption is done by using a parametrized model for the HWIs. Here, equal ADC bit resolution is assumed and the results show that a Massive MIMO setup with 4 or 5 bit per ADC, maximizes the SE under a power consumption constraint that, in turn, can be tuned to achieve maximal EE. Second, the case of allowing different ADC bit resolutions in each RF chain is considered under a fixed sum of ADC bits among all the BS antennas. Majorization theory is used to study the order preserving properties of the $\mathrm{SE}$ and the power consumption under i.i.d. Rayleigh fading. The results show that maximal SE and minimum power consumption is attained when all ADC bits are equal across the BS antennas. Finally, the optimal ADC bit allocation to minimize the pilot distortion, maximize the SINR or minimize the power consumption is studied under correlated Rayleigh fading with LSF variations among the UL channels towards different BS antennas. The results show that equal $\mathrm{ADC}$ bit allocation is close to optimal in terms of $\mathrm{SE}$ for cases with low LSF variations among the UL channels towards different BS antennas. In contrast, when there are large LSF channel variation among $\mathrm{BS}$ antennas, such as in cell-free Massive MIMO, optimizing the ADC bit allocation combined with interference suppression techniques can provide high SE gains compared to the Massive MIMO baseline with equal ADC bit allocation. In terms of EE, for the case of cell-free Massive MIMO, equal ADC bit allocation provides higher EE compared to mixed-ADCs when minimizing the power consumption under a product of SINRs constraint. On the other hand, when the product of SINRs is maximized under a power consumption constraint, mixed-ADCs with RZF offer higher EE compared to equal ADC bit allocation.

In conclusion, the baseline Massive MIMO design, when properly optimized, supports a high SE and EE. This design is the preferred choice in scenarios with co-located BS antenna arrays and where the number of samples in the coherence block is much larger than number of spatially multiplexed UEs. The SP alternative design, when combined with data-aided channel estimation methods (e.g., MICED), can offer improvements in SE over the 
Massive MIMO baseline in cases with short coherence block sizes. On the other hand, the mixed-ADCs alternative design can increase SE and EE compared to the Massive MIMO baseline in cases with distributed BS antennas (e.g., cell-free Massive MIMO) when interference suppression techniques are used (e.g., RZF).

The alternative Massive MIMO designs explored in this dissertation can improve the SE and EE performance of Massive MIMO compared to its baseline operation. However, this improvements come at the cost of adding more complexity into the system. Thus, an interesting topic for further research is within the implementation of these alternative designs in practical testbeds to obtain more accurate insights into the balance between complexity and performance.

Another path for future research directions would be to study the joint design of the many parts that constitute a communication system, to achieve a global optimization of the network. For example, in this dissertation it was shown that the MICED algorithm can improve the SE, however, the design of channel codes was left out of the analysis. Thus, it would be interesting to combine the properties of Massive MIMO within the design of channel codes. Furthermore, the scheduling of data traffic in higher layers of the communication protocol stack can be adapted so that the benefits of spatial multiplexing can be used to the fullest. 
4 Summary, Conclusions, and Future Work 


\section{Bibliography}

[1] Cisco, "Visual networking index: Global mobile data traffic forecast update, 2017-2022,” Tech. Rep., Feb. 2019. [Online]. Available: https: //www.cisco.com/c/en/us/solutions/collateral/service-provider/ visual-networking-index-vni/white-paper-c11-738429.pdf

[2] Ericsson, "Ericsson mobility report," Tech. Rep., Nov. 2019. [Online]. Available: https://www.ericsson.com/4a517b/assets/local/ mobility-report/documents/2019/emr-q2-2019-update.pdf

[3] D. Aziz, K. Kusume, O. Queseth, and et la., "D8.4: METIS final project report,” ICT-317669-METIS, Tech. Rep., Apr. 2015. [Online]. Available: https://metis2020.com/wp-content/uploads/deliverables/ METIS_D8.4_v1.pdf

[4] W. V. Heddeghem, S. Lambert, B. Lannoo, D. Colle, M. Pickavet, and P. Demeester, "Trends in worldwide ICT electricity consumption from 2007 to 2012," Computer Commun., vol. 50, pp. 64-76, Feb. 2014.

[5] A. Fehske, G. Fettweis, J. Malmodin, and G.Biczok, "The global footprint of mobile communications: The ecological and economic perspective," IEEE Trans. Commun., vol. 49, no. 8, pp. 55-62, Aug. 2011.

[6] X. Zhang and J. G. Andrews, "Downlink cellular network analysis with multi-slope path loss models," IEEE Trans. Commun., vol. 63, no. 5, pp. 1881-1894, May 2015.

[7] T. L. Marzetta, E. G. Larsson, H. Yang, and H. Q. Ngo, Fundamentals of Massive MIMO. Cambridge Press, 2016.

[8] E. Björnson, J. Hoydis, and L. Sanguinetti, "Massive MIMO networks: Spectral, energy, and hardware efficiency," Foundations and Trends ${ }^{\circledR}$ in Signal Processing, vol. 11, no. 3-4, pp. 154-655, Nov. 2017. 
[9] J. Vinogradova, E. Björnson, and E. G. Larsson, “On the separability of signal and interference-plus-noise subspaces in blind pilot decontamination," in Proc. IEEE ICASSP, Mar. 2016, pp. 3421-3425.

[10] H. Q. Ngo and E. G. Larsson, "EVD-based channel estimation in multicell multiuser MIMO systems with very large antenna arrays," in Proc. IEEE ICASSP, Mar. 2012, pp. 3249-3252.

[11] R. R. Müller, L. Cottatellucci, and M. Vehkaperä, "Blind pilot decontamination,” IEEE J. Sel. Topics Signal Process., vol. 8, no. 5, pp. 773-786, Oct. 2014.

[12] H. Huh, G. Caire, H. C. Papadopoulos, and S. A. Ramprashad, "Achieving "massive MIMO" spectral efficiency with a not-so-large number of antennas," IEEE Trans. Wireless Commun., vol. 11, no. 9, pp. 3226-3239, Sep. 2012.

[13] H. Yin, D. Gesbert, M. Filippou, and Y. Liu, "A coordinated approach to channel estimation in large-scale multiple-antenna systems," IEEE J. Sel. Areas Commun., vol. 31, no. 2, pp. 264-273, Feb. 2013.

[14] E. Björnson, J. Hoydis, and L. Sanguinetti, "Massive MIMO has unlimited capacity," IEEE Trans. Wireless Commun., vol. 17, no. 1, pp. 574-590, Jan 2018.

[15] H. Yang and T. L. Marzetta, "Total energy efficiency of cellular large scale antenna system multiple access mobile networks," in Proc. IEEE OnlineGreenComm, Oct. 2013, pp. 27-32.

[16] Y. Li, Y.-H. Nam, B. L. Ng, and J.Zhang, "A non-asymptotic throughput for massive MIMO cellular uplink with pilot reuse," in Proc. IEEE GLOBECOM, Dec. 2012, pp. 4500-4504.

[17] E. Björnson, E. Larsson, and M. Debbah, "Massive MIMO for maximal spectral efficiency: How many users and pilots should be allocated?" IEEE Trans. Wireless Commun., vol. 15, no. 2, pp. 1293-1308, Feb. 2016.

[18] R. Mochaourab, E. Björnson, and M. Bengtsson, "Adaptive pilot clustering in heterogeneous massive MIMO networks," IEEE Trans. Wireless Commun., vol. 15, no. 8, pp. 5555-5568, Aug. 2016.

[19] P. Hoeher and F. Tufvesson, "Channel estimation with superimposed pilot sequence," in Proc. IEEE GLOBECOM, Dec. 1999, pp. 2162-2166. 
[20] G. T. Zhou, M. Viberg, and T. McKelvey, "A first-order statistical method for channel estimation," IEEE Signal Process. Lett., vol. 10, no. 3, pp. 57-60, Mar. 2003.

[21] M. Dong, L. Tong, and B. M. Sadler, "Optimal insertion of pilot symbols for transmissions over time-varying flat fading channels," IEEE Trans. Signal Process., vol. 52, no. 5, pp. 1403-1418, May 2004.

[22] A. T. Asyhari and S. ten Brink, "Orthogonal or superimposed pilots? a rate-efficient channel estimation strategy for stationary MIMO fading channels," IEEE Trans. Wireless Commun., vol. 16, no. 5, pp. 2776-2789, May 2017.

[23] K. Upadhya, S. A. Vorobyov, and M. Vehkaperä, "Superimposed pilots are superior for mitigating pilot contamination in massive MIMO," IEEE Trans. Signal Process., vol. 65, no. 11, pp. 2917-2932, Jun. 2017.

[24] K. Upadhya, S. A. Vorobyov, and M. Vehkaperä, "Downlink performance of superimposed pilots in massive MIMO systems," IEEE Trans. Wireless Commun., vol. 17, no. 10, pp. 6630-6644, Oct 2018.

[25] H. Zhang, S. Gao, D. Li, H. Chen, and L. Yang, "On superimposed pilot for channel estimation in multicell multiuser MIMO uplink: Large system analysis," IEEE Trans. Veh. Technol., vol. 65, no. 3, pp. 1492-1505, Mar. 2016.

[26] H. Zhu, B. Farhang-Boroujeny, and C. Schlegel, "Pilot embedding for joint channel estimation and data detection in MIMO communication systems," IEEE Commun. Letters, vol. 7, no. 1, pp. 30-32, Jan 2003.

[27] M. A. Khalighi and S. Bourennane, "Semiblind single-carrier MIMO channel estimation using overlay pilots," IEEE Trans. Veh. Technol., vol. 57, no. 3, pp. 1951-1956, May 2008.

[28] J. Ma and L. Ping, "Data-aided channel estimation in large antenna systems," IEEE Trans. Signal Process., vol. 62, no. 12, pp. 3111-3124, Jun 2014.

[29] J. Ma, C. Liang, C. Xu, and L. Ping, "On orthogonal and superimposed pilot schemes in massive MIMO NOMA systems," IEEE J. Sel Areas Commun., vol. 35, no. 12, pp. 2696-2707, Dec 2017. 
[30] C. Han, T. Harrold, S. Armour, I. Krikidis, S. Videv, P. M. Grant, H. Haas, J. S. Thompson, I. Ku, C. Wang, T. A. Le, M. R. Nakhai, J. Zhang, and L. Hanzo, "Green radio: radio techniques to enable energy-efficient wireless networks," IEEE Commun. Mag., vol. 49, no. 6, pp. 46-54, Jun. 2011.

[31] Z. Hasan, H. Boostanimehr, and V. K. Bhargava, "Green cellular networks: A survey, some research issues and challenges," IEEE Commun. Surveys Tuts., vol. 13, no. 4, pp. 524-540, Nov. 2011.

[32] E. Björnson, J. Hoydis, M. Kountouris, and M. Debbah, "Massive MIMO systems with non-ideal hardware: Energy efficiency, estimation, and capacity limits," IEEE Trans. Inf. Theory, vol. 60, no. 11, pp. 7112-7139, Nov. 2014.

[33] E. Björnson, M. Matthaiou, and M. Debbah, "Massive MIMO with nonideal arbitrary arrays: Hardware scaling laws and circuit-aware design," IEEE Trans. Wireless Commun., vol. 14, no. 8, pp. 4353-4368, Aug. 2015.

[34] C. Desset and L. V. der Perre, "Validation of low-accuracy quantization in massive MIMO and constellation EVM analysis," in Proc. EuCNC, Jun. 2015, pp. 21-25.

[35] C. Studer and G. Durisi, "Quantized massive MU-MIMO-OFDM uplink,” IEEE Trans. Commun., vol. 64, no. 6, pp. 2387-2399, Jun. 2016.

[36] S. Jacobsson, G. Durisi, M. Coldrey, U. Gustavsson, and C. Studer, "Throughput analysis of massive MIMO uplink with low-resolution ADCs," IEEE Trans. Wireless Commun., vol. 16, no. 6, pp. 4038-4051, Jun. 2017.

[37] C. Mollén, J. Choi, E. G. Larsson, and R. W. Heath Jr., "Achievable uplink rates for massive MIMO with coarse quantization," in Proc. IEEE ICASSP, Mar. 2017.

[38] C. Mollén, J. Choi, E. G. Larsson, and R. W. Heath Jr., "Uplink performance of wideband massive MIMO with one-bit ADCs," IEEE Trans. Wireless Commun., vol. 16, no. 1, pp. 87-100, Jan. 2017.

[39] A. Mezghani, N. Damak, and J. A. Nossek, "Circuit aware design of power-efficient short range communication systems," in Proc. IEEE ISWCS, Sep. 2010, pp. 869-873. 
[40] O. Orhan, E. Erkip, and S. Rangan, "Low power analog-to-digital conversion in millimeter wave systems: Impact of resolution and bandwidth on performance," in Proc. IEEE ITA, Feb. 2015, pp. 191-198.

[41] N. Liang and W. Zhang, "Mixed-ADC massive MIMO," IEEE J. Sel. Areas Commun., vol. 34, no. 4, pp. 983-997, Apr. 2016.

[42] T. Zhang, C. Wen, S. Jin, and T. Jiang, "Mixed-ADC massive MIMO detectors: Performance analysis and design optimization," IEEE Trans. Wireless Commun., vol. 15, no. 11, pp. 7738-7752, Nov. 2016.

[43] I. Z. Ahmed, H. Sadjadpour, and S. Yousefi, "Capacity analysis and bit allocation design for variable-resolution ADCs in massive MIMO," in Proc. IEEE MILCOM, Oct 2018, pp. 1-6.

[44] H. Pirzadeh and A. L. Swindlehurst, "Spectral efficiency of mixed-ADC massive MIMO,” IEEE Trans. Signal Process., vol. 66, no. 13, pp. 3599-3613, July 2018.

[45] A. W. Marshall, I. Olkin, and B. C. Arnold, Inequalities: theory of majorization and its applications. Springer New York, 2011.

[46] E. Jorswieck and H. Boche, "Majorization and matrix-monotone functions in wireless communications," Foundations and Trends ${ }^{\circledR}$ in Communications and Information Theory, vol. 3, no. 6, pp. 553-701, Jul 2007. [Online]. Available: http://dx.doi.org/10.1561/ 0100000026

[47] E. F. W. Alexanderson, "Transoceanic radio communication," Trans. American Inst. Electr. Eng., vol. XXXVIII, no. 2, pp. 1269-1285, Jul. 1919.

[48] L. R. Kahn, "Ratio squarer," Proc. IRE, vol. 42, no. 11, p. 1704, 1954.

[49] D. G. Brennan, "On the maximal signal-to-noise ratio realizable from several noisy signals," Proc. IRE, vol. 43, no. 10, p. 1530, 1955.

[50] --, "Linear diversity combining techniques," Proc. IRE, vol. 47, no. 6, pp. 1075-1102, Jun. 1959.

[51] J. H. Winters, "Optimum combining in digital mobile radio with cochannel interference," IEEE Trans. Veh. Technol., vol. 33, no. 3, pp. 144-155, Aug. 1984. 
[52] J. Winters, "Optimum combining for indoor radio systems with multiple users," IEEE Trans. Commun., vol. 35, no. 11, pp. 1222-1230, Nov. 1987.

[53] S. C. Swales, M. A. Beach, D. J. Edwards, and J. P. McGeehan, “The performance enhancement of multibeam adaptive base-station antennas for cellular land mobile radio systems," IEEE Trans. Veh. Technol., vol. 39, no. 1, pp. 56-67, Feb. 1990.

[54] S. Anderson, M. Millnert, M. Viberg, and B. Wahlberg, "An adaptive array for mobile communication systems," IEEE Trans. Veh. Technol., vol. 40, no. 1, pp. 230-236, Feb. 1991.

[55] R. H. Roy III. and B. Ottersten, "Spatial division multiple access wireless communication systems,” Patent, Dec., 1991.

[56] P. Zetterberg and B. Ottersten, "The spectrum efficiency of a base station antenna array system for spatially selective transmission," IEEE Trans. Veh. Technol., vol. 44, no. 3, pp. 651-660, Aug. 1995.

[57] S. Anderson, U. Forssen, J. Karlsson, T. Witzschel, P. Fischer, and A. Krug, "Ericsson/Mannesmann GSM field-trials with adaptive antennas," in Proc. IEEE VTC, vol. 3, May. 1997, pp. 1587-1591.

[58] S. Anderson, B. Hagerman, H. Dam, U. Forssen, J. Karlsson, F. Kronestedt, S. Mazur, and K. J. Molnar, "Adaptive antennas for GSM and TDMA systems," IEEE Pers. Commun., vol. 6, no. 3, pp. 74-86, Jun. 1999.

[59] G. J. Foschini and M. J. Gans, "On limits of wireless communications in a fading environment when using multiple antennas," Springer Wireless Personal Commun., vol. 6, pp. 311-335, Mar. 1998.

[60] I. E. Telatar, "Capacity of multi-antenna Gaussian channels," European Trans. Telecommun., vol. 10, pp. 585-595, Dec. 1999.

[61] M. Medard, "The effect upon channel capacity in wireless communications of perfect and imperfect knowledge of the channel," IEEE Trans. Inf. Theory, vol. 46, no. 3, pp. 933-946, May 2000.

[62] G. Caire and S. Shamai, "On the achievable throughput of a multiantenna Gaussian broadcast channel," IEEE Trans. Inf. Theory, vol. 49, no. 7, pp. 1691-1706, Jul. 2003. 
[63] S. Vishwanath, N. Jindal, and A. Goldsmith, "Duality, achievable rates, and sum-rate capacity of Gaussian MIMO broadcast channels," IEEE Trans. Inf. Theory, vol. 49, no. 10, pp. 2658-2668, Oct. 2003.

[64] P. Viswanath and D. N. C. Tse, "Sum capacity of the vector Gaussian broadcast channel and uplink-downlink duality," IEEE Trans. Inf. Theory, vol. 49, no. 8, pp. 1912-1921, Aug. 2003.

[65] T. L. Marzetta, "Noncooperative cellular wireless with unlimited numbers of base station antennas," IEEE Trans. Wireless Commun., vol. 9, no. 11, pp. 3590-3600, 2010.

[66] S. Parkvall, E. Dahlman, A. Furuskar, and M. Frenne, "NR: the new 5g radio access technology," IEEE Commun. Stand. Mag., vol. 1, no. 4, pp. 24-30, Dec. 2017.

[67] Sprint, "Continued progress on next-gen network plan," Tech. Rep., Jan. 2019. [Online]. Available: https://newsroom.sprint.com/ fyq318-network-progress.htm

[68] E. G. Larsson and E. Björnson, "Massive MIMO news - commentary - mythbusting," Tech. Rep. [Online]. Available: http://www. massive-mimo.net

[69] D. Tse and P. Viswanath, Fundamentals of wireless Communication. Cambridge Press, 2005.

[70] A. J. Jerri, "The shannon sampling theorem \#8212; its various extensions and applications: A tutorial review," Proceedings of the IEEE, vol. 65, no. 11, pp. 1565-1596, Nov. 1977.

[71] E. Björnson and L. Sanguinetti, "Demystifying the power scaling law of intelligent reflecting surfaces and metasurfaces," 2019. [Online]. Available: https://arxiv.org/abs/1908.03133

[72] X. Gao, O. Edfors, F. Rusek, and F. Tufvesson, "Massive MIMO performance evaluation based on measured propagation data," IEEE Trans. Wireless Commun., vol. 14, no. 7, pp. 3899-3911, Jul. 2015.

[73] L. Sanguinetti, E. Björnson, and J. Hoydis, “Towards massive MIMO 2.0: Understanding spatial correlation, interference suppression, and pilot contamination," IEEE Trans. Commun., pp. 1-1, 2019.

[74] J. Vieira, F. Rusek, O. Edfors, S. Malkowsky, L. Liu, and F. Tufvesson, "Reciprocity calibration for massive MIMO: Proposal, modeling, 
and validation," IEEE Trans. Wireless Commun., vol. 16, no. 5, pp. 3042-3056, Mar. 2017.

[75] H. Q. Ngo and E. G. Larsson, "No downlink pilots are needed in TDD massive MIMO," IEEE Trans. Wireless Commun., vol. 16, no. 5, pp. 2921-2935, May 2017.

[76] F. Sohrabi and W. Yu, "Hybrid digital and analog beamforming design for large-scale antenna arrays," IEEE J. Sel. Topics Signal Process., vol. 10, no. 3, pp. 501-513, Apr. 2016.

[77] T. E. Bogale, L. B. Le, A. Haghighat, and L. Vandendorpe, "On the number of RF chains and phase shifters, and scheduling design with hybrid analog-digital beamforming," IEEE Trans. Wireless Commun., vol. 15, no. 5, pp. 3311-3326, May 2016.

[78] R. W. Heath Jr., N. Gonzalez-Prelcic, S. Rangan, W. Roh, and A. M. Sayeed, "An overview of signal processing techniques for millimeter wave MIMO systems," IEEE J. Sel. Topics Signal Process., vol. 10, no. 3, pp. 436-453, Mar. 2016.

[79] N. Tawa, T. Kuwabara, Y. Maruta, M. Tanio, and T. Kaneko, “28 GHz downlink multi-user MIMO experimental verification using 360 element digital AAS for 5G Massive MIMO,” in Proc. EuMC, 2018.

[80] S. M. Kay, Fundamentals of Statistical Signal Processing: Estimation Theory. Prentice Hall, 1993.

[81] H. Weingarten, Y. Steinberg, and S. S. Shamai, "The capacity region of the Gaussian multiple-input multiple-output broadcast channel," IEEE Trans. Inf. Theory, vol. 52, no. 9, pp. 3936-3964, Sep. 2006.

[82] Z. Chen and E. Björnson, "Channel hardening and favorable propagation in cell-free massive MIMO with stochastic geometry," IEEE Trans. Commun., vol. 66, no. 11, pp. 5205-5219, Nov 2018.

[83] E. Björnson, L. Sanguinetti, J. Hoydis, and M. Debbah, “Optimal design of energy-efficient multi-user MIMO systems: Is massive MIMO the answer?" IEEE Trans. Wireless Commun., vol. 14, no. 6, pp. 3059-3075, Jun. 2015.

[84] B. Murmann, "ADC performance survey 1997-2018," [Online]. Available: http://web.stanford.edu/ murmann/adcsurvey.html. 
[85] B. Murmann, "Limits on ADC power dissipation," in Analog Circuit Design, A. H. M. Roermund and J. v. Huijsing, Eds. Springer, 2006.

[86] A. Mezghani and J. A. Nossek, "Modeling and minimization of transceiver power consumption in wireless networks," in Proc. IEEE ITG WSA, Feb. 2011, pp. 1-8.

[87] J. Max, "Quantizing for minimum distortion," IEEE Trans. Inf. Theory, vol. 6, no. 1, pp. 7-12, Mar. 1960.

[88] C. Mollén, J. Choi, E. G. Larsson, and R. W. Heath Jr., "Uplink performance of wideband massive MIMO with one-bit ADCs," IEEE Trans. Wireless Commun., vol. 16, no. 1, pp. 87-100, Jan. 2017.

[89] E. Björnson, E. Jorswieck, M. Debbah, and B. Ottersten, "Multiobjective signal processing optimization: The way to balance conflicting metrics in 5g systems," IEEE Signal Process. Mag., vol. 31, no. 6, pp. 14-23, Nov. 2014.

[90] E. Björnson and E. Jorswieck, "Optimal resource allocation in coordinated multi-cell systems," Foundations and Trends ${ }^{\circledR}$ in Communications and Information Theory, vol. 9, no. 2-3, pp. 113-381, 2013. [Online]. Available: http://dx.doi.org/10.1561/ 0100000069

[91] S. Boyd and L. Vandenberghe, Convex Optimization. Cambridge Press, 2004.

[92] S. Boyd, S.-J. Kim, L. Vandenberghe, and A. Hassibi, "A tutorial on geometric programming," Optim. Eng., vol. 8, no. 1, pp. 67-127. [Online]. Available: https://web.stanford.edu/ boyd/papers/pdf/ gp_tutorial.pdf

[93] M. Grant and S. Boyd, "Graph implementations for nonsmooth convex programs," in Recent Advances in Learning and Control, ser. Lecture Notes in Control and Information Sciences, V. Blondel, S. Boyd, and H. Kimura, Eds. Springer-Verlag Limited, 2008, pp. 95-110. [Online]. Available: https://web.stanford.edu/ boyd/papers/pdf/ graph_dcp.pdf

[94] --, "CVX: Matlab software for disciplined convex programming, version 2.1," http://cvxr.com/cvx, Mar. 2014.

[95] D. J. C. MacKay, Information Theory, Inference \& Learning Algorithms. New York, NY, USA: Cambridge University Press, 2002. 
[96] P. Sweeney, Error Control Coding: From Theory to Practice. New York, NY, USA: John Wiley \& Sons, Inc., 2002.

[97] "NR multiplexing and channel coding (release 15)," Tech. Rep., 2018, 3GPP TS 38.212.

[98] J. J. Bussgang, "Crosscorrelation functions of amplitude-distorted Gaussian signals," Research Laboratory of Electronics, Massachusetts Institute of Technology, Tech. Rep. 216, Mar. 1952.

[99] E. Björnson, L. Sanguinetti, and J. Hoydis, "Hardware distortion correlation has negligible impact on UL massive MIMO spectral efficiency," IEEE Trans. Commun., vol. 67, no. 2, pp. 1085-1098, Feb. 2019.

[100] U. Gustavsson, C. Sanchez-Perez, T. Eriksson, F. Athley, G. Durisi, P. Landin, K. Hausmair, C. Fager, and L. Svensson, "On the impact of hardware impairments on massive MIMO," in Proc. IEEE GLOBECOM, Dec. 2014, pp. 294-300.

[101] A. Mezghani, M. Rouatbi, and J. A. Nossek, "An iterative receiver for quantized MIMO systems," in Proc. IEEE MELECON, Mar. 2012, pp. 1049-1052. 


\section{Included Papers}




\section{Papers}

The papers associated with this thesis have been removed for copyright reasons. For more details about these see:

http://urn.kb.se/resolve?urn=urn:nbn:se:liu:diva-163131 



\section{Other Recently Published Theses From \\ The Division of Communication Systems Department of Electrical Engineering (ISY) Linköping University, Sweden}

Trịnh Văn Chiến, Spatial Resource Allocation in Massive MIMO Communications: From Cellular to Cell-Free, Linköping Studies in Science and Technology. Dissertations, No. 2036, 2020.

Amin Ghazanfari, Power Control for Multi-Cell Massive MIMO, Linköping Studies in Science and Technology. Licentiate Thesis, No. 1852, 2019.

Giovanni Interdonato, Signal Processing Aspects of Cell-Free Massive MIMO, Linköping Studies in Science and Technology. Licentiate Thesis, No. 1817, 2018.

Marcus Karlsson, Blind Massive MIMO Base Stations: Downlink Transmission and Jamming, Linköping Studies in Science and Technology. Dissertations, No. 1950, 2018.

Hei Victor Cheng, Optimizing Massive MIMO: Precoder Design and Power Allocation, Linköping Studies in Science and Technology. Dissertations, No. 1929, 2018.

Christopher Mollén, High-End Performance with Low-End Hardware: Analysis of Massive MIMO Base Station Transceivers, Linköping Studies in Science and Technology. Dissertations, No. 1896, 2017.

Trịnh Văn Chiến, Resource Allocation for Max-Min Fairness in Multi-Cell Massive MIMO, Linköping Studies in Science and Technology. Licentiate Thesis, No. 1797, 2017.

Victor Hei Cheng, Aspects of Power Allocation in Massive MIMO, Linköping Studies in Science and Technology. Licentiate Thesis, No. 1767, 2016.

Marcus Karlsson, Aspects of Massive MIMO, Linköping Studies in Science and Technology. Licentiate Thesis, No. 1764, 2016.

Christopher Mollén, On Massive MIMO Base Stations with Low-End Hardware, Linköping Studies in Science and Technology. Licentiate Thesis, No. 1756, 2016.

Antonios Pitarokoilis, Phase Noise and Wideband Transmission in Massive MIMO, Linköping Studies in Science and Technology. Dissertations, No. 1756, 2016.

Anu Kalidas M. Pillai, Signal Reconstruction Algorithms for Time-Interleaved ADCs, Linköping Studies in Science and Technology. Dissertations, No. 1672, 2015. 
Ngô Quốc Hiển, Massive MIMO: Fundamentals and System Designs, Linköping Studies in Science and Technology. Dissertations, No. 1642, 2015.

Mirsad Čirkić, Efficient MIMO Detection Methods, Linköping Studies in Science and Technology. Dissertations, No. 1570, 2014.

Reza Moosavi, Improving the Efficiency of Control Signaling in Wireless Multiple Access Systems, Linköping Studies in Science and Technology. Dissertations, No. 1556, 2014.

Johannes Lindblom, The MISO Interference Channel as a Model for Non-Orthogonal Spectrum Sharing, Linköping Studies in Science and Technology. Dissertations, No. 1555, 2014.

Antonios Pitarokoilis, On the Performance of Massive MIMO Systems with Single Carrier Transmission and Phase Noise, Linköping Studies in Science and Technology. Licentiate Thesis, No. 1618, 2013.

Tumula V. K. Chaitanya, HARQ Systems: Resource Allocation, Feedback Error Protection, and Bits-to-Symbol Mappings, Linköping Studies in Science and Technology. Dissertations, No. 1555, 2013. 


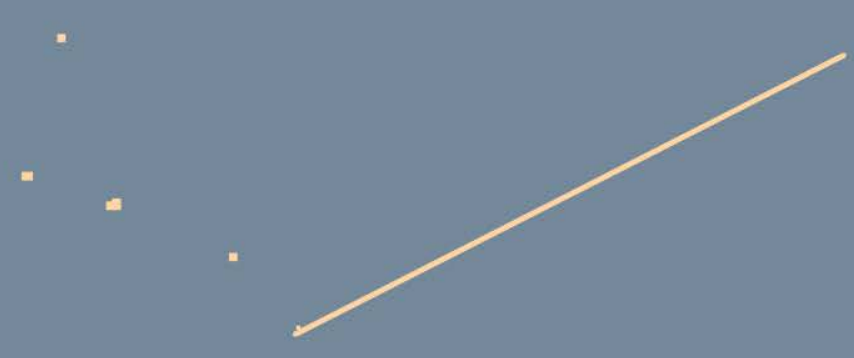

\section{FACULTY OF SCIENCE AND ENGINEERING}

Linköping Studies in Science and Technology Dissertations, No. 2041, 2020

Division of Communication Systems

Department of Electrical Engineering

Linköping University

SE-581 83 Linköping, Sweden

\section{www.liu.se}

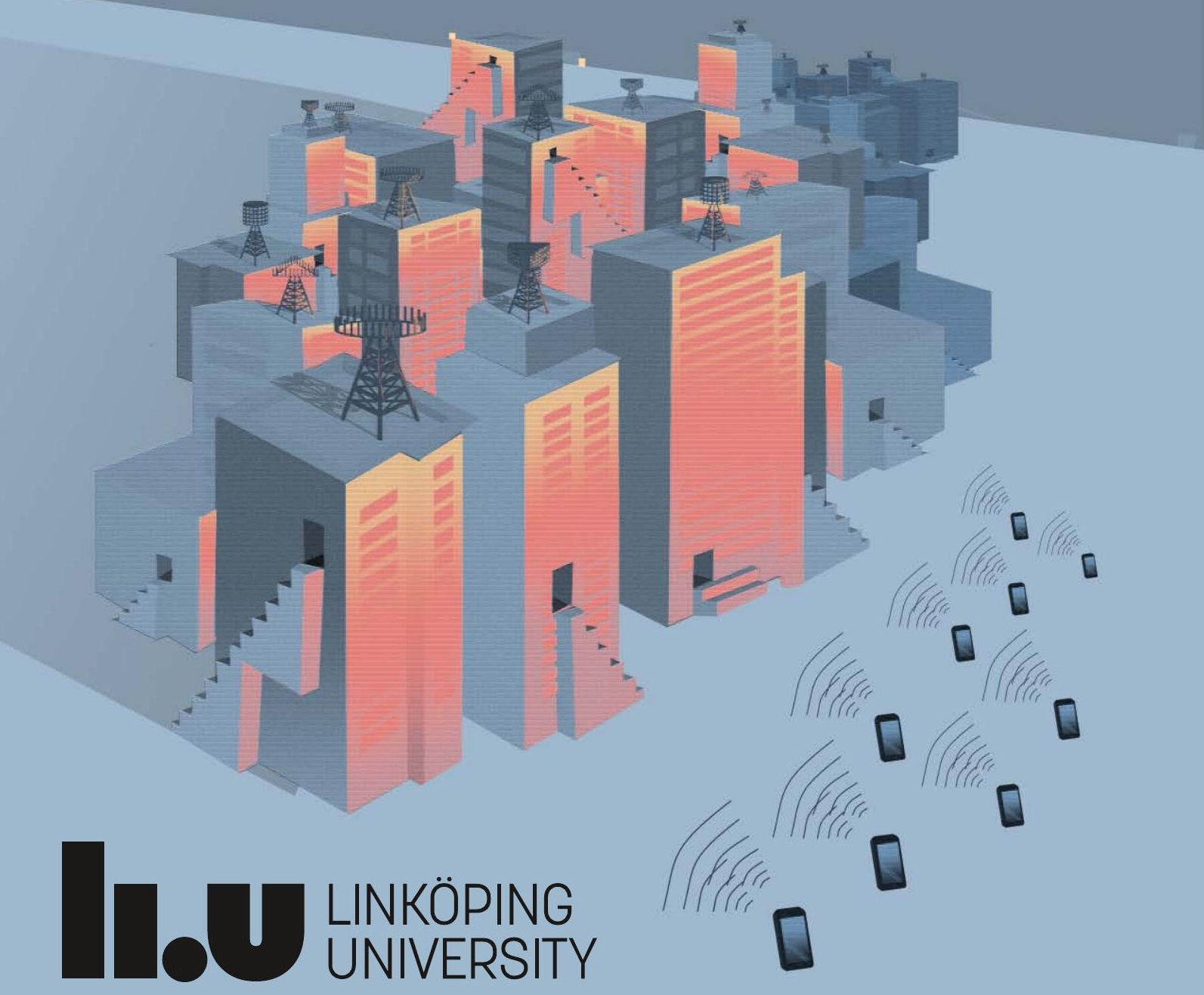

